\title{
A MINIMAX METHOD FOR FINDING SADDLE CRITICAL POINTS OF UPPER SEMI-DIFFERENTIABLE LOCALLY LIPSCHITZ CONTINUOUS FUNCTIONAL IN HILBERT SPACE AND ITS CONVERGENCE
}

\begin{abstract}
XUDONG YAO
AbStract. A minimax characterization for finding nonsmooth saddle critical points, i.e., saddle critical points of locally Lipschitz continuous functional, in Banach space is presented in [X. Yao and J. Zhou, A local minimax characterization for computing multiple nonsmooth saddle critical points, Math. Program., 104 (2005), no. 2-3, Ser. B, 749-760]. By this characterization, a descent-max method is devised. But, there is no numerical experiment and convergence result for the method. In this paper, to a class of locally Lipschitz continuous functionals, a minimax method for computing nonsmooth saddle critical points in Hilbert space will be designed. Numerical experiments will be carried out and convergence results will be established.
\end{abstract}

\section{INTRODUCTION}

Let $B$ be a Banach space, $B^{*}$ its dual space, $\langle$,$\rangle the dual relation, and \|\cdot\|$ its norm. To a locally Lipschitz continuous functional $J: B \rightarrow \mathbb{R}$, the generalized gradient $\partial J(u)$ at $u \in B$ in the sense of Clarke [7] is defined as follows.

Definition 1.1. Let $J$ be Lipschitz continuous near $u_{0} \in B$. The generalized directional derivative $J^{0}\left(u_{0}, v\right)$ of $J$ at $u_{0}$ in the direction of $v \in B$ is defined by

$$
J^{0}\left(u_{0}, v\right)=\limsup _{\substack{u \rightarrow u_{0} \\ t \downarrow 0}} \frac{J(u+t v)-J(u)}{t} .
$$

The generalized gradient $\partial J\left(u_{0}\right)$ of $J$ at $u_{0}$ is a subset of $B^{*}$ given by

$$
\partial J\left(u_{0}\right)=\left\{\zeta \in B^{*} \mid\langle\zeta, v\rangle \leq J^{0}\left(u_{0}, v\right), \forall v \in B\right\} .
$$

To convex functionals, we have the following definition for the subgradient.

Definition 1.2. Let $J: B \rightarrow \mathbb{R}$ be convex. The subgradient $\partial J\left(u_{0}\right)$ of $J$ at $u_{0}$ is a subset of $B^{*}$ given by

$$
\partial J\left(u_{0}\right)=\left\{\zeta \in B^{*} \mid\left\langle\zeta, u-u_{0}\right\rangle \leq J(u)-J\left(u_{0}\right), \forall u \in B\right\} .
$$

For convex functionals, the generalized gradient coincides with the subgradient.

According to Chang [5], a point $u_{0} \in B$ is a critical point of a locally Lipschitz continuous functional $J$ if and only if

$$
0 \in \partial J\left(u_{0}\right) .
$$

Received by the editor July 23, 2010 and, in revised form, October 23, 2011.

2010 Mathematics Subject Classification. Primary 65K10, 65K15, 65N12; Secondary 49M37.

Key words and phrases. Locally Lipschitz continuous functional, nonsmooth saddle critical point, minimax method, convergence. 
If $J$ is a $C^{1}$ functional, $\partial J\left(u_{0}\right)=\left\{\nabla J\left(u_{0}\right)\right\}$, i.e., $0 \in \partial J\left(u_{0}\right)$ becomes $\nabla J\left(u_{0}\right)=0$, the well-known Euler-Lagrange equation. Critical points of a $C^{1}$ functional are called smooth critical points and critical points of a locally Lipschitz continuous functional are called nonsmooth critical points.

The classical optimization theory, nonsmooth analysis and calculus of variation study local maxima or minima. Traditional numerical methods in these areas are for finding local extremum points. Local extremum points are critical points. A critical point $u_{0}$ of $J$, that is not a local extremum point, is a saddle point, i.e., $u_{0}$ is a critical point and in any neighborhood $\mathcal{N}\left(u_{0}\right)$ of $u_{0}$, there are $v, w \in \mathcal{N}\left(u_{0}\right)$ such that

$$
J(v)<J\left(u_{0}\right)<J(w) .
$$

In physical systems, saddle points appear as unstable equilibria or transient excited states.

The minimax principle, which characterizes a saddle point of $J$ as a solution to

$$
\min _{A \in \mathcal{A}} \max _{v \in A} J(v)
$$

for some collection $\mathcal{A}$ of subsets $A$ in $B$, is one of the most popular approaches in critical point theory. For smooth critical points, the Mountain Pass Lemma established in 1973 by Ambrosetti and Rabinowitz [2] set a milestone in contemporary critical point theory. Then, various saddle point theorems and linking theorems were established in the literature to prove existence of multiple critical points for various nonlinear problems; see [3], 12]. For nonsmooth critical points, in 1981, Chang [5] introduced the notion of nonsmooth critical points and obtained a nonsmooth version of the saddle point theorem of Rabinowitz. Kourogenis and Papageorgiou [9] generalized Chang's results and Kandilakis, Kourogenis and Papageorgiou [8] obtained a nonsmooth version of the Linking Theorem. All these saddle point theorems and linking theorems in the literature focus on the existence issue and they are not helpful to devise numerical algorithms for finding saddle critical points. For computing smooth saddle critical points, Li and Zhou [10] established a local minimax characterization of smooth saddle critical points in Hilbert space and designed a minimax algorithm based on the characterization. Then, Yao and Zhou [14] extended the local minimax characterization and the minimax algorithm in Hilbert space to a local minimax characterization and a minimax algorithm in Banach space. These two algorithms were successfully carried out to find smooth saddle critical points and convergence results were established; see [10, [11, 14, [15]. In 2005, Yao and Zhou [13] gave a local minimax characterization for nonsmooth saddle critical points and devised a descent-max method by the characterization. But, there is no numerical experiment and convergence result for the method. In this paper, to a class of locally Lipschitz continuous functionals, the local minimax characterization for nonsmooth saddle critical points in [13] will be reestablished in Hilbert space in another way. Then, a minimax method for finding nonsmooth saddle critical points will be designed. Numerical experiments will be carried out and convergence results will be obtained.

A typical example on application of nonsmooth critical point theory to partial differential equations is the Dirichlet problem,

$$
\left\{\begin{array}{l}
-\Delta u(x)=f(x, u(x)), x \in \Omega, \\
\left.u(x)\right|_{x \in \partial \Omega}=0
\end{array}\right.
$$


where $\Omega$ is an open bounded domain in $\mathbb{R}^{n}$ with smooth boundary $\partial \Omega, f(x, t)$ is a measurable function defined on $\Omega \times \mathbb{R}$ and for every $x \in \Omega, f(x, t)$ is locally bounded. The corresponding variational functional is

$$
J(u)=\frac{1}{2} \int_{\Omega}|\nabla u(x)|^{2} d x-\int_{\Omega} F(x, u(x)) d x,
$$

where $F(x, t)=\int_{0}^{t} f(x, s) d s$. Denote $G(u)=\int_{\Omega} F(x, u(x)) d x$. Then, according to Chang [5], we have the following theorems for the problem (1.1).

Theorem 1.3. If $f(x, t)$ satisfies

$$
|f(x, t)| \leq C_{1}+C_{2}|t|^{\sigma}
$$

for $x \in \Omega \subset \mathbb{R}^{n}$ and $t \in \mathbb{R}$, where $0<\sigma<\frac{n+2}{n-2}$ for $n \geq 3, \sigma>0$ for $n=1,2$ and $C_{1}, C_{2}>0$ are two constants, then, $G(u)=\int_{\Omega} \int_{0}^{u(x)} f(x, s) d s d x$ is locally Lipschitz continuous in $L^{\sigma+1}(\Omega)$ and $H_{0}^{1}(\Omega)$ as well. In addition, if $\bar{f}(x, t)$ and $\hat{f}(x, t)$ are $N$-measurable (cf. 4), where

$$
\bar{f}(x, t)=\lim _{\delta \rightarrow 0} \operatorname{ess} \inf _{|s-t|<\delta} f(x, s)
$$

and

$$
\hat{f}(x, t)=\lim _{\delta \rightarrow 0} \operatorname{ess} \sup _{|s-t|<\delta} f(x, s),
$$

then

$$
\{\zeta(x) \mid \zeta \in \partial G(u)\} \subseteq[\bar{f}(x, u(x)), \hat{f}(x, u(x))] \text { a.e. }
$$

in $L^{\sigma+1}(\Omega)$ and $H_{0}^{1}(\Omega)$ as well.

Remark 1.4. It is just for simplicity to assume $\sigma>0$ for $n=1,2$ in Theorem 1.3, Theorem 1.5] Lemma 3.6, and for $m=1,2$ in Lemma 4.20, Lemma 4.21.

Theoretically, for the Dirichlet problem (1.1), people will find $u_{0} \in H_{0}^{1}(\Omega)$ such that

$$
-\Delta u_{0}(x) \in\left[\bar{f}\left(x, u_{0}(x)\right), \hat{f}\left(x, u_{0}(x)\right)\right]
$$

for all $x \in \Omega[5]$. By the inclusion $\{\zeta(x) \mid \zeta \in \partial G(u)\} \subseteq[\bar{f}(x, u(x)), \hat{f}(x, u(x))]$ in Theorem 1.3 to find such $u_{0}$, we can find a critical point of $J$. But, from a numerical point of view, this inclusion offers little information on $\partial G(u)$ and it is not helpful for computing critical points of $J$. Also in [5], Chang sharpened the conclusion.

Theorem 1.5. If $f(x, t)$ is a Baire-measurable function defined on $\Omega \times \mathbb{R}$, is nondecreasing in $t$, and satisfies

$$
|f(x, t)| \leq C_{1}+C_{2}|t|^{\sigma}
$$

for $x \in \Omega \in \mathbb{R}^{n}$ and $t \in \mathbb{R}$, where $0<\sigma<\frac{n+2}{n-2}$ for $n \geq 3, \sigma>0$ for $n=1,2$ and $C_{1}, C_{2}>0$ are two constants, then the functional $G(u)=\int_{\Omega} \int_{0}^{u(x)} f(x, s) d s d x$ is convex and

$\partial G(u)=\{\zeta: \Omega \rightarrow \mathbb{R} \mid \zeta$ is measurable, $\zeta(x) \in[f(x, u(x)-0), f(x, u(x)+0)] \forall x \in \Omega\}$ in $L^{\sigma+1}(\Omega)$ and $H_{0}^{1}(\Omega)$ as well.

Remark 1.6. The conclusions in this theorem and in Theorem 2.3 in 5 ] look different. If we read the proof of Theorem 2.3 in [5] carefully, it will be found that indeed Chang verified the conclusion in this theorem. 
The equality

$\partial G(u)=\{\zeta: \Omega \rightarrow \mathbb{R} \mid \zeta$ is measurable, $\zeta(x) \in[f(x, u(x)-0), f(x, u(x)+0)] \forall x \in \Omega\}$ is much better than the inclusion

$$
\{\zeta(x) \mid \zeta \in \partial G(u)(x)\} \subseteq[\bar{f}(x, u(x)), \hat{f}(x, u(x))] .
$$

It says that finding $u_{0} \in H_{0}^{1}(\Omega)$ which satisfies (1.2) is equivalent to finding a critical point of $J$. On the other hand, this equality offers clear information on $\partial G(u)$ and it is helpful for calculating critical points of $J$. In Theorem 1.5

$$
J(u)=I(u)-G(u),
$$

where $I(u)=\frac{1}{2} \int_{\Omega}|\nabla u(x)|^{2} d x \in C^{1}\left(H_{0}^{1}(\Omega), \mathbb{R}\right)$ and $G(u)=\int_{\Omega} \int_{0}^{u(x)} f(x, s) d s d x$ is convex on $H_{0}^{1}(\Omega)$. In this paper, upper semi-differentiable locally Lipschitz continuous functional will be defined in Banach space $B$ first in Section 2. A locally Lipschitz continuous functional

$$
J(u)=I(u)-G(u), \quad \forall u \in B,
$$

where $I \in C^{1}(B, \mathbb{R})$ and $G: B \rightarrow \mathbb{R}$ is convex, is an upper semi-differentiable locally Lipschitz continuous functional on $B$. Then, to upper semi-differentiable locally Lipschitz continuous functionals, the local minimax characterization for nonsmooth saddle critical points in [13] will be reestablished in Hilbert space $H$ and, according to this local minimax characterization, a minimax algorithm for capturing saddle critical points of upper semi-differentiable locally Lipschitz continuous functionals will be presented. In Section 3, the SC-condition will be defined first. Based on the SC-condition, the subsequence and sequence convergence for the minimax algorithm will be established. This is the first time that convergence results are obtained for a minimax algorithm to capture nonsmooth saddle points. In Section 4, this minimax algorithm will be implemented to solve numerical examples.

At the end of this section, let us recall some simple properties of the generalized directional derivative $J^{0}(u, v)$ and the generalized gradient $\partial J(u)$ to locally Lipschitz continuous functional $J$.

Proposition $1.7([7])$. Assume that $J, W$ are locally Lipschitz continuous in $B$. $B^{*}$.

(a) For every $u \in B, \partial J(u)$ is a nonempty, convex and $w^{*}$-compact subset of

(b) If $|J(w)-J(v)| \leq K\|w-v\|$ for all $w, v$ in a neighborhood of $u \in B$, then $\partial J(u)$ is a bounded set in $B^{*}$ with bound $K$.

(c) $\partial(J+W)(u) \subseteq \partial J(u)+\partial W(u)$ for every $u \in B$ and for $\lambda \in \mathbb{R}, \partial(\lambda J)(u)=$ $\lambda \partial J(u)$ for every $u \in B$.

(d) If $u \in B$ is a local minimum or maximum point of $J$, then $0 \in \partial J(u)$.

(e) (Chain Rule) Let $X$ be a Banach space. If $L: X \rightarrow B$ is strictly differential at $v$, i.e., there is a $D_{s} L(v) \in \mathcal{L}(X, B)$ such that for each $w \in X$,

$$
\lim _{\substack{v^{\prime} \rightarrow v \\ t \downarrow 0}} \frac{L\left(v^{\prime}+t w\right)-L\left(v^{\prime}\right)}{t}=\left\langle D_{s} L(v), w\right\rangle
$$

and the convergence is uniform for $w$ in compact sets, then $F=J \circ L$ has

$$
\partial F(v) \subset \partial J(L(v)) \circ D_{s} L(v) .
$$

Equality holds if $L$ maps every neighborhood of $v$ to a set which is dense in a neighborhood of $L(v)$. 
(f) $J^{0}(u, v): B \times B \rightarrow \mathbb{R}$ is upper semicontinuous.

\section{A minimax method in Hilbert space}

Let $H$ be a Hilbert space, $\langle$,$\rangle the inner product, \|\cdot\|$ the norm introduced by the inner product $\langle$,$\rangle and L$ a closed subspace of $H$. Denote $[L, v]$ as the subspace spanned by $L$ and $v \in H$ and $\left[u_{1}, u_{2}, \ldots, u_{m}\right]$ as the subspace spanned by $u_{i} \in H$, $i=1,2, \ldots, m$. For any subspace $H^{\prime}$ of $H, S_{H^{\prime}}$ is the unit sphere in $H^{\prime}$ and for any closed subspace $M$ of $H, H=M \oplus M^{\perp}$ is the orthogonal decomposition of $H$ and $M^{\perp}$ is the orthogonal complement of $M$ in $H$. For any $v \in H$ and $U \subset H$, $d(v, U)=\inf _{u \in U}\|v-u\|$, i.e., the distance between $v$ and $U$.

To design a minimax algorithm, the following peak selection is important.

Definition 2.1. A set-valued mapping $P: S_{L^{\perp}} \rightarrow 2^{H}$ is the peak mapping of a locally Lipschitz continuous functional $J: H \rightarrow \mathbb{R}$ w.r.t. $L$ if $\forall v \in S_{L^{\perp}}, P(v)=$ $\{u \in[L, v]: u$ is a local maximum point of $J \operatorname{in}[L, v]\}$. A single-valued mapping $p: S_{L^{\perp}} \rightarrow H$ is a peak selection of the locally Lipschitz continuous functional $J$ w.r.t. $L$ if $p(v) \in P(v), \forall v \in S_{L^{\perp}}$. For a given $v \in S_{L^{\perp}}$, if $p$ is locally defined in a neighborhood of $v$, we say that $J$ has a local peak selection $p$ at $v$.

The peak selections have the following property.

Lemma 2.2. Assume that $L \subset H$ is a finite dimensional space, $v \in S_{L^{\perp}}$ and $p$ is a local peak selection of a locally Lipschitz continuous functional $J$ w.r.t. $L$ at $v$. Then, there is $z \in \partial J(p(v))$ such that $z \perp[L, v]$.

Proof. Since $L$ is a finite dimension space, assume that $u_{1}, u_{2}, \ldots, u_{m}$ is a basis. Consider the composite functional

$$
F\left(t_{0}, t_{1}, \ldots, t_{m}\right)=J\left(t_{0} v+t_{1} u_{1}+\cdots+t_{m} u_{m}\right) .
$$

According to (e) in Proposition 1.7 we have

$$
\partial F\left(t_{0}, t_{1}, \ldots, t_{m}\right)=\left\{\left(\langle\zeta, v\rangle,\left\langle\zeta, u_{1}\right\rangle, \ldots,\left\langle\zeta, u_{m}\right\rangle\right) \mid \zeta \in \partial J\left(t_{0} v+t_{1} u_{1}+\cdots+t_{m} u_{m}\right)\right\} .
$$

Since $p$ is a peak selection, by $(\mathrm{d})$ in Proposition 1.7, $0 \in \partial F\left(t_{0}^{v}, t_{1}^{v}, \ldots, t_{m}^{v}\right)$, where $p(v)=t_{0}^{v} v+t_{1}^{v} u_{1}+\cdots+t_{m}^{v} u_{m}$, i.e., there is $z \in \partial J(p(v))$ such that

$$
\langle z, v\rangle=0 \text { and }\left\langle z, u_{i}\right\rangle=0, \quad i=1, \ldots, m \text {, }
$$

i.e., $z \perp[L, v]$.

Remark 2.3. This conclusion is simple and important. To upper semi-differentiable locally Lipschitz continuous functionals which will be defined later, it will be used to reestablish the local minimax characterization for nonsmooth saddle critical points in 13 in Hilbert space. On the other hand, if $J \in C^{1}(H, \mathbb{R}), \partial J(p(v))=$ $\{\nabla J(p(v))\}$ and $\nabla J(p(v)) \perp[L, v]$ are obviously true.

We give two simple lemmas which will be used later.

Lemma 2.4. In Banach space $B$, for any point $v$ with $\|v\|=1$, it holds that

$$
\left\|v-\frac{v+w}{\|v+w\|}\right\| \leq \frac{2\|w\|}{\|v+w\|}, \quad \forall w \in B
$$


Proof. Since $\|v\|=1$, we have

Lemma 2.5. For

$$
\begin{aligned}
\left\|v-\frac{v+w}{\|v+w\|}\right\| & =\left\|\frac{(\|v+w\|-1) v+w}{\|v+w\|}\right\| \leq \frac{|\|v+w\|-1|\|v\|+\|w\|}{\|v+w\|} \\
& =\frac{|\|v+w\|-\|v\||+\|w\|}{\|v+w\|} \leq \frac{2\|w\|}{\|v+w\|} .
\end{aligned}
$$

$$
J(u)=I(u)-G(u), \quad \forall u \in B,
$$

where $I \in C^{1}(B, \mathbb{R})$ and $G: B \rightarrow \mathbb{R}$ is locally Lipschitz continuous on Banach space $B$,

$$
\partial J(u)=\{\nabla I(u)-g \mid g \in \partial G(u)\},
$$

where $\nabla I$ is the gradient of $I$.

Proof. By (c) in Proposition 1.7.

$$
\partial J(u) \subseteq \partial I(u)+\partial(-G)(u)=\{\nabla I(u)\}-\partial G(u),
$$

i.e.,

$$
\partial J(u) \subseteq\{\nabla I(u)-g \mid g \in \partial G(u)\} .
$$

On the other hand, by (c) in Proposition 1.7 again,

$$
\partial G(u) \subseteq \partial I(u)+\partial(-J)(u)=\{\nabla I(u)\}-\partial J(u) .
$$

This means that $\nabla I(u)-g_{0} \in \partial J(u)$ for every $g_{0} \in \partial G(u)$. Then,

$$
\left\{\nabla I(u)-g_{0} \mid g_{0} \in \partial G(u)\right\} \subseteq \partial J(u) .
$$

Hence,

$$
\partial J(u)=\{\nabla I(u)-g \mid g \in \partial G(u)\} .
$$

In this section, we will establish a minimax characterization for upper semidifferentiable locally Lipschitz continuous functionals. First, we define upper and lower semi-differentiable locally Lipschitz continuous functionals.

Definition 2.6. In Banach space $B$, a locally Lipschitz continuous functional $J$ is an upper semi-differentiable locally Lipschitz continuous functional if, for every $u \in B, z \in \partial J(u)$ implies, for $w$ around $u$,

$$
J(w)-J(u) \leq\langle z, w-u\rangle+F(w ; u, z),
$$

where $F(w ; u, z)=o(\|w-u\|)$ is an upper-bound functional around $u$ and a locally Lipschitz continuous functional $I$ is a lower semi-differentiable locally Lipschitz continuous functional if, for every $u \in B, z \in \partial I(u)$ implies, for $w$ around $u$,

$$
I(w)-I(u) \geq\langle z, w-u\rangle+G(w ; u, z),
$$

where $G(w ; u, z)=o(\|w-u\|)$ is a lower-bound functional around $u$ and $\langle$,$\rangle is the$ dual relation between $B$ and its dual space $B^{*}$.

Remark 2.7. (a) If $J: B \rightarrow \mathbb{R}$ is Fréchet differentiable, then, to every $u \in B$, $z \in \partial J(u)=\{\nabla J(u)\}$ implies

$$
J(w)-J(u)=\langle z, w-u\rangle+o(\|w-u\|)
$$

for $w$ around $u$, i.e.,

$$
J(w)-J(u) \leq\langle z, w-u\rangle+o(\|w-u\|)
$$

for $w$ around $u$, and

$$
J(w)-J(u) \geq\langle z, w-u\rangle+o(\|w-u\|)
$$


for $w$ around $u$. To a locally Lipschitz continuous functional $J: B \rightarrow \mathbb{R}$, if, for every $u \in B, z \in \partial J(u)$ implies both (2.1) and (2.2), then $J$ is Fréchet differentiable. Hence, a locally Lipschitz continuous functional $J: B \rightarrow \mathbb{R}$ can be considered as a semi-differentiable locally Lipschitz continuous functional if, for every $u \in B$, $z \in \partial J(u)$ implies (2.1) or for every $u \in B, z \in \partial J(u)$ implies (2.2).

(b) If $J \in C^{1}(B, \mathbb{R}), J$ is an upper semi-differentiable locally Lipschitz continuous functional and also a lower semi-differentiable locally Lipschitz continuous functional.

The following lemma gives us an important class of upper semi-differentiable locally Lipschitz continuous functionals.

Lemma 2.8. A locally Lipschitz continuous functional

$$
J(u)=I(u)-G(u), \quad \forall u \in B,
$$

where $I \in C^{1}(B, \mathbb{R})$ and $G: B \rightarrow \mathbb{R}$ is convex on Banach space $B$, is an upper semi-differentiable locally Lipschitz continuous functional on $B$.

Proof. Since $I \in C^{1}(B, \mathbb{R})$, to every $u \in B$,

$$
I(w)-I(u)=\langle\nabla I(u), w-u\rangle+o(\|w-u\|)
$$

for $w$ around $u$ and since $G$ is convex in $B$, to every $u \in B$ and $g \in \partial G(u)$,

$$
G(w)-G(u) \geq\langle g, w-u\rangle
$$

for $w \in B$. By Lemma 2.5, for $z \in \partial J(u)$, there is $g_{0} \in \partial G(u)$ such that $z=$ $\nabla I(u)-g_{0}$. Thus,

$$
\begin{aligned}
& J(w)-J(u)=(I(w)-I(u))-(G(w)-G(u)) \\
\leq & \left\langle\nabla I(u)-g_{0}, w-u\right\rangle+o(\|w-u\|)=\langle z, w-u\rangle+o(\|w-u\|)
\end{aligned}
$$

for $w$ around $u$, i.e., $J$ is a upper semi-differentiable locally Lipschitz continuous functional on $B$.

Remark 2.9. According to the verification of the lemma, it is clear that

$$
F(w ; u, z)=I(w)-I(u)-\langle\nabla I(u), w-u\rangle=o(\|w-u\|)
$$

is an upper-bound functional of $J$ around $u$.

To simplify the statement of mathematical justification and convergence results for our minimax algorithm, we need the definition for super-linear property of peak selection.

Definition 2.10. Let $J$ be an upper semi-differentiable locally Lipschitz continuous functional on Hilbert space $H$ and $H=L \oplus L^{\perp}$ for a finite dimensional subspace $L \subset H$. If $p$ is a local peak selection of $J$ w.r.t. $L$ at $v \in S_{L^{\perp}}$ such that $p$ is continuous at $v$ and, for every $z \in \partial J(p(v)) \cap[L, v]^{\perp}$, there is an upper-bound functional $F(u ; p(v), z)$ of $J$ around $p(v)$ satisfying

$$
\lim _{w \rightarrow v} \frac{|F(p(w) ; p(v), z)|}{\|w-v\|}=0,
$$

we say that the peak selection $p$ has super-linear property at $v$. If $p$ is a peak selection of $J$ w.r.t. $L$ defined on an open set $V \subset H$ and $p$ has super-linear property at every $v \in V$, we say that the peak selection $p$ has super-linear property in $V$. 
Remark 2.11. (a) Theoretically, we can give a general definition by using "for a $z \in$ $\partial J(p(v)) \cap[L, v]^{\perp}$ " instead of "for every $z \in \partial J(p(v)) \cap[L, v]^{\perp}$ ". If we choose that special $z$ in the general definition as $z$ in Lemma 2.14, then, we still have Lemma 2.14 and Theorem 2.16. We can still use Lemma 2.14 as step-size rule to design a minimax algorithm. But, numerically, we prefer "for every $z \in \partial J(p(v)) \cap[L, v]^{\perp}$ " since it is hard to find that special $z$ in numerical computation. On the other hand, for establishing convergence results, i.e., Theorem 3.9, Theorem 3.12 and Corollary 3.14, we also prefer "for every $z \in \partial J(p(v)) \cap[L, v]^{\perp}$ ".

(b) Since $F(p(w) ; p(v), z)=o(\|p(w)-p(v)\|)$, the Lipschitz continuity of $p$ around $v$, i.e.,

$$
\|p(w)-p(v)\| \leq l\|w-v\|
$$

for $w$ around $v$, where $l>0$ is a constant, means that the peak selection $p$ has super-linear property at $v$. Indeed, usually the Hölder continuity of $p$ around $v$, i.e.,

$$
\|p(w)-p(v)\| \leq l\|w-v\|^{\alpha}
$$

for $w$ around $v$, where $l>0$ is a constant and $0<\alpha \leq 1$ is close to 1 , will guarantee that the peak selection $p$ has super-linear property at $v$.

For $J \in C^{1}(H, \mathbb{R})$, we will prove that continuity of peak selection $p$ of $J$ w.r.t. a finite dimensional subspace $L \subset H$ at $v \in S_{L^{\perp}}$ implies super-linear property of $p$ at $v$. First, we verify a simple and useful lemma.

Lemma 2.12. Assume that $H$ is a Hilbert space and $v \in H$ such that $\|v\|=1$. Denote $M=\{t v \mid t \in \mathbb{R}\}$. Then, for every $u \in H$ with $\|u\|=1$ and $\langle u, v\rangle>0$, there is unique $d \in M^{\perp}$ with $\|d\|=1$ and $s \geq 0$ such that

$$
u=\frac{1}{\sqrt{1+s^{2}}}(v-s d) \text {. }
$$

Proof. Since $H$ is a Hilbert space, there is $w \in M^{\perp}$ with $\|w\|=1$ such that

$$
u=c_{1} v+c_{2} w \text {. }
$$

Then, by $\|u\|=\|v\|=\|w\|=1$ and $w \in M^{\perp}$, we know

$$
c_{1}^{2}+c_{2}^{2}=1 \text {. }
$$

On the other hand, $\langle u, v\rangle>0,\|v\|=1$ and $w \in M^{\perp}$ mean $c_{1}=\frac{\langle u, v\rangle}{\|v\|^{2}}=\langle u, v\rangle>0$. Thus, there is $s \geq 0$ such that $c_{1}=\frac{1}{\sqrt{1+s^{2}}}$. If we set

$$
d=\left\{\begin{array}{cl}
w, & \text { if } c_{2} \leq 0 \\
-w, & \text { if } c_{2}>0
\end{array}\right.
$$

then, by (2.3) and (2.4),

$$
u=\frac{1}{\sqrt{1+s^{2}}}(v-s d) .
$$

Since $c_{2} w$ is unique and $c_{2}=\langle u, w\rangle, d$ is unique.

By Lemma 2.12, we establish a relation between continuity and super-linear property of peak selection of $J \in C^{1}(H, \mathbb{R})$ as follows.

Lemma 2.13. Assume that $H$ is a Hilbert space, $J \in C^{1}(H, \mathbb{R}), H=L \oplus L^{\perp}$ for a finite dimensional subspace $L \subset H$ and $p$ is a local peak selection of $J$ w.r.t. $L$ at $v \in S_{L^{\perp}}$. If $p$ is continuous at $v$, then $p$ has super-linear property at $v$. 
Proof. In Hilbert space $L^{\perp}$, if $w \in S_{L^{\perp}}$ and $\|w-v\|<1$, then

$$
1>\|w-v\|^{2}=\|w\|^{2}+\|v\|^{2}-2\langle w, v\rangle=2-2\langle w, v\rangle, \text { i.e., }\langle w, v\rangle>\frac{1}{2} .
$$

Thus, by Lemma 2.12, for every $w \in S_{L^{\perp}}$ with $\|w-v\|<1$, there is $s \geq 0$ and $d \in[L, v]^{\perp}$ such that $\|d\|=1$ and

$$
w=\frac{1}{\sqrt{1+s^{2}}}(v+s d)
$$

Hence, for small $s>0$,

$$
\begin{aligned}
& J(p(w))-J(p(v))=J\left(p\left(\frac{v+s d}{\|v+s d\|}\right)\right)-J(p(v)) \\
\leq & J\left(p\left(\frac{v+s d}{\|v+s d\|}\right)\right)-J\left(\frac{t_{w}}{\|v+s d\|} v+w_{L}\right)=\frac{t_{w} s}{\|v+s d\|}\langle\nabla J(\eta), d\rangle \\
= & \frac{t_{w} s}{\|v+s d\|}(\langle\nabla J(p(v)), d\rangle+\langle\nabla J(\eta)-\nabla J(p(v)), d\rangle) \\
= & \langle\nabla J(p(v)), p(w)-p(v)\rangle+\frac{t_{w} s}{\|v+s d\|}\langle\nabla J(\eta)-\nabla J(p(v)), d\rangle,
\end{aligned}
$$

where $p(w)=t_{w} \frac{v+s d}{\|v+s d\|}+w_{L}, w_{L} \in L, \eta=\lambda p(w)+(1-\lambda)\left(\frac{t_{w}}{\|v+s d\|} v+w_{L}\right)$ and $\lambda \in[0,1]$, i.e.,

$$
|F(p(w) ; p(v), \nabla J(p(v)))| \leq \frac{\left|t_{w}\right| s}{\|v+s d\|}|\langle\nabla J(\eta)-\nabla J(p(v)), d\rangle|,
$$

where

$$
\begin{aligned}
& F(u ; p(v), \nabla J(p(v))) \\
= & \begin{cases}D(u ; p(v), \nabla J(p(v))), & \text { if } D(u ; p(v), \nabla J(p(v)))>0, \\
0, & \text { if } D(u ; p(v), \nabla J(p(v))) \leq 0\end{cases}
\end{aligned}
$$

is an upper-bound functional of $J$ around $p(v)$ and

$$
D(u ; p(v), \nabla J(p(v)))=J(u)-J(p(v))-\langle\nabla J(p(v)), u-p(v)\rangle .
$$

On the other hand,

$$
\begin{aligned}
& \|w-v\|=\left\|\frac{1}{\sqrt{1+s^{2}}}(v+s d)-v\right\|=\left\|\left(\frac{1}{\sqrt{1+s^{2}}}-1\right) v+\frac{s}{\sqrt{1+s^{2}}} d\right\| \\
= & \frac{1}{\sqrt{1+s^{2}}}\left\|\left(1-\sqrt{1+s^{2}}\right) v+s d\right\|=\frac{s}{\sqrt{1+s^{2}}}\left\|\frac{s}{1+\sqrt{1+s^{2}}} v-d\right\| \\
= & \frac{s}{\sqrt{1+s^{2}}} \sqrt{1+\frac{s^{2}}{\left(1+\sqrt{1+s^{2}}\right)^{2}}},
\end{aligned}
$$

i.e.,

$$
\lim _{w-v} \frac{\|w-v\|}{s}=1
$$

Hence, by (2.5) and (2.6),

$$
\lim _{w \rightarrow v} \frac{|F(p(w) ; p(v), \nabla J(p(v)))|}{\|w-v\|}=0,
$$

i.e., $p$ has super-linear property at $v$. 
By peak selection, the following lemma can be verified. The conclusion will be used as a step-size rule for our minimax algorithm.

Lemma 2.14. Let $H$ be a Hilbert space with $H=L \oplus L^{\perp}$ for a finite dimensional subspace $L \subset H$. Assume that $J$ is a upper semi-differentiable locally Lipschitz continuous functional and $p$ is a local peak selection of $J$ w.r.t. $L$ at $v \in S_{L^{\perp}}$ such that $p(v)$ is not a critical point, $p$ has super-linear property at $v$ and $d(p(v), L)>0$. Choose $z \in \partial J(p(v)) \cap[L, v]^{\perp}$. Denote $p(v)=t_{v} v+w_{v}$, where $w_{v} \in L$ and $w=$ $-\operatorname{sign}\left(t_{v}\right) \frac{z}{\|z\|}$. Then, as $s>0$ is small,

$$
J(p(v(s)))-J(p(v))<-\frac{1}{4} s\left|t_{v}\right|\|z\|,
$$

where $v(s)=\frac{v+s w}{\|v+s w\|}=\frac{1}{\sqrt{1+s^{2}}}(v+s w)$.

Proof. Since $J$ is a upper semi-differentiable locally Lipschitz continuous functional and $p$ is continuous at $v$, we have

$$
J(p(v(s)))-J(p(v)) \leq\langle z, p(v(s))-p(v)\rangle+F(p(v(s)) ; p(v), z)
$$

for small $s>0$, where $F(p(v(s)) ; p(v), z)=o(\|p(v(s))-p(v)\|)$ is any upper-bound functional around $p(v)$. On the other hand, since $z \perp[L, v]$,

$$
\langle z, p(v)\rangle=\left\langle z, t_{v} v+w_{v}\right\rangle=0
$$

and

$$
\begin{gathered}
\langle z, p(v(s))\rangle=\left\langle z, t_{s} v(s)+w_{s}\right\rangle \\
=\left\langle z, t_{s} \frac{v+s w}{\|v+s w\|}+w_{s}\right\rangle=-\frac{\operatorname{sign}\left(t_{v}\right) t_{s} s\|z\|}{\|v+s w\|},
\end{gathered}
$$

where $p(v(s))=t_{s} v(s)+w_{s}$ and $w_{s} \in L$. According to the continuity of $p$ at $v$ and $d(p(v), L)>0$,

$$
\operatorname{sign}\left(t_{v}\right)=\operatorname{sign}\left(t_{s}\right)
$$

for small $s>0$. Since $\|v\|=1$,

$$
\|v+s w\| \leq 2
$$

for small $s>0$. Thus, we have

$$
\begin{aligned}
J(p(v(s)))-J(p(v)) & \leq-\frac{\operatorname{sign}\left(t_{v}\right) t_{s} s\|z\|}{\|v+s w\|}+F(p(v(s)) ; p(v), z) \\
& \leq-\frac{1}{2} s\left|t_{v}\right|\|z\|+F(p(v(s)) ; p(v), z)
\end{aligned}
$$

for small $s>0$. By super-linear property of $p$ at $v$, there is a upper-bound functional $F_{0}(p(v(s)) ; p(v), z)$ around $p(v)$ such that

$$
\lim _{v(s) \rightarrow v} \frac{\left|F_{0}(p(v(s)) ; p(v), z)\right|}{\|v(s)-v\|}=0 .
$$

Then, by (2.6),

$$
\lim _{s \rightarrow 0} \frac{\left|F_{0}(p(v(s)) ; p(v), z)\right|}{s}=0 .
$$

Therefore, since $p(v)$ is not a critical point and $d(p(v), L)>0$, as $s>0$ is small,

$$
J(p(v(s)))-J(p(v))<-\frac{1}{4} s\left|t_{v}\right|\|z\| .
$$


Remark 2.15. (a) The existence of $z \in \partial J(p(v)) \cap[L, v]^{\perp}$ is guaranteed by Lemma 2.2 .

(b) Comparing Lemma 2.14 with Lemma 3 in [13, the difference is different elements of generalized gradient are used to decide descent direction. In this lemma, $z \in \partial J(p(v))$ with $z \perp[L, v]$ is used. In Lemma 3 in [13], $z \in \partial J(p(v))$ with $\|z\|=\min \{\|\zeta\| \zeta \in \in \partial J(p(v))\}$ is used. If $p$ is continuous at $v \in S_{L^{\perp}}$, for a sequence of generalized gradients $\left\{z_{n}\right\}$ such that $z_{n} \in \partial J\left(p\left(v_{n}\right)\right) \cap\left[L, v_{n}\right]^{\perp},\left\{v_{n}\right\} \subset S_{L^{\perp}}$, $z_{n} \rightarrow z$ and $v_{n} \rightarrow v$, we have $z \in \partial J(p(v)) \cap[L, v]^{\perp}$. This property is crucial to the convergence of our minimax algorithm. For a sequence of generalized gradients $\left\{z_{n}\right\}$ such that $\left\|z_{n}\right\|=\min \left\{\|\zeta\| \zeta \in \partial J\left(p\left(v_{n}\right)\right)\right\},\left\{v_{n}\right\} \subset S_{L^{\perp}}, z_{n} \rightarrow z$ and $v_{n} \rightarrow v$, we do have $z \in \partial J(p(v))$ under the assumption that $p$ is continuous at $v \in S_{L^{\perp}}$. But, we do not know if $\|z\|=\min \{\|\zeta\| \mid \zeta \in \partial J(p(v))\}$.

(c) Comparing this lemma with Lemma 2.1 in [10, we assume super-linear property of $p$ at $v$ here instead of continuity of $p$ at $v$ in Lemma 2.1 in [10]. By Lemma 2.13 , if $J \in C^{1}(H, \mathbb{R})$, these two assumptions are equivalent.

By Lemma 2.14, a local minimax characterization for nonsmooth saddle critical points in 13. can be reestablished to upper semi-differentiable locally Lipschitz continuous functionals as follows.

Theorem 2.16. Let $H$ be a Hilbert space with $H=L \oplus L^{\perp}$ for a finite dimensional subspace $L \subset H$. Assume that $J$ is a upper semi-differentiable locally Lipschitz continuous functional and $p$ is a local peak selection of $J$ w.r.t. $L$ at $v \in S_{L^{\perp}}$ such that

(a) $p$ has super-linear property at $v$ and $d(p(v), L)>0$, and

(b) $v$ is a local minimum point of $J(p(\cdot))$ on $S_{L^{\perp}}$.

Then, $p(v)$ is a critical point of $J$.

Proof. If $p(v)$ is not a critical point of $J$, then by Lemma 2.14, as $s>0$ is small,

$$
J(p(v(s)))-J(p(v))<-\frac{1}{4} s\left|t_{v}\right|\|z\|,
$$

where $v(s)=\frac{v+s w}{\|v+s w\|}, w=-\operatorname{sign}\left(t_{v}\right) \frac{z}{\|z\|}, z \in \partial J(p(v)) \cap[L, v]^{\perp}, p(v)=t_{v} v+w_{v}$ and $w_{v} \in L$. It is a contradiction to assumption (b).

Remark 2.17. (a) Suppose that a critical point $u$ is characterized by Theorem 2.16 . Then, $u=p(v)$ means, for any neighborhood $\mathcal{N}(u)$ of $u$, there is $u_{1} \in[L, v]$ such that $u_{1} \in \mathcal{N}(u)$ and $J\left(u_{1}\right)<J(u)$ except that $J$ is a constant functional around $u$ on $[L, v]$, and, $u=p(v)$ and $v$ is a local minimum point of $J(p(\cdot))$ on $S_{L^{\perp}}$ mean, in any neighborhood $\mathcal{N}(u)$ of $u$, there is $v_{2} \in S_{L^{\perp}}$ around $v$ such that $u_{2}=p\left(v_{2}\right) \in \mathcal{N}(u)$ and $J\left(u_{2}\right)=J\left(p\left(v_{2}\right)\right)>J(p(v))=J(u)$ except that $J(p(\cdot))$ is a constant functional around $v$ on $S_{L^{\perp}}$. Therefore, except extreme cases, in any neighborhood $\mathcal{N}(u)$ of $u=p(v)$, there are $u_{1}, u_{2} \in \mathcal{N}(u)$ such that $J\left(u_{1}\right)<J(u)<J\left(u_{2}\right)$, i.e., $u$ is a saddle point of $J$.

(b) Comparing this theorem with Theorem 2.1 in [10, we assume super-linear property of $p$ at $v$ in this theorem instead of continuity of $p$ at $v$ in Theorem 2.1 in [10]. By Lemma 2.13, if $J \in C^{1}(H, \mathbb{R})$, these two assumptions are equivalent.

According to Lemma 2.14 and Theorem 2.16, we can design a minimax algorithm for capturing saddle critical points of an upper semi-differentiable locally Lipschitz continuous functional $J$. Assume that $u_{1}, u_{2}, \ldots, u_{n-1}$ are found critical points, 
$L=\left[u_{1}, u_{2}, \ldots, u_{n-1}\right], \lambda>0$ is a constant and $\varepsilon$ is a small positive number. The following is the flow chart for the algorithm.

Step 1. Choose $v_{n}^{1} \in S_{L^{\perp}}$.

Step 2. Set $k=1$ and solve for

$$
\begin{aligned}
& u_{n}^{k}=p\left(v_{n}^{k}\right)=t_{0}^{k} v_{n}^{k}+t_{1}^{k} u_{1}+\cdots+t_{n-1}^{k} u_{n-1} \\
= & \arg \max \left\{J\left(t_{0} v_{n}^{k}+t_{1} u_{1}+\cdots+t_{n-1} u_{n-1}\right) \mid t_{i} \in \mathbb{R}, i=0,1, \ldots, n-1\right\} .
\end{aligned}
$$

Step 3. Find a descent direction $w_{n}^{k}=-\operatorname{sign}\left(t_{0}^{k}\right) \frac{z_{n}^{k}}{\left\|z_{n}^{k}\right\|}$ at $u_{n}^{k}$, where $z_{n}^{k} \in \partial J\left(u_{n}^{k}\right) \cap$ $\left[L, v_{n}^{k}\right]^{\perp}$.

Step 4. If $\left\|z_{n}^{k}\right\|<\varepsilon$, then output $u_{n}^{k}$, stop. Otherwise, do Step 5.

Step 5. For each $s>0$, let $v_{n}^{k}(s)=\frac{v_{n}^{k}+s w_{n}^{k}}{\left\|v_{n}^{k}+s w_{n}^{k}\right\|}$ and use initial point $\left(t_{0}^{k}, t_{1}^{k}, \ldots, t_{n-1}^{k}\right)$ to solve for

$p\left(v_{n}^{k}(s)\right)=\arg \max \left\{J\left(t_{0} v_{n}^{k}(s)+\sum_{i=1}^{n-1} t_{i} u_{i}\right) \mid t_{i} \in \mathbb{R}, i=0,1, \ldots, n-1\right\}$,

then set $v_{n}^{k+1}=v_{n}^{k}\left(s_{n}^{k}\right)$ and $u_{n}^{k+1}=p\left(v_{n}^{k+1}\right)=t_{0}^{k+1} v_{n}^{k+1}+t_{1}^{k+1} u_{1}+\cdots+$ $t_{n-1}^{k+1} u_{n-1}$, where $s_{n}^{k}$ satisfies

$s_{n}^{k}=\max \left\{s=\frac{\lambda}{2^{m}}\left|m \in \mathbb{N}, J\left(p\left(v_{n}^{k}(s)\right)\right)-J\left(p\left(v_{n}^{k}\right)\right) \leq-\frac{1}{4}\right| t_{0}^{k}|s| \mid z_{n}^{k} \|\right\}$.

Step 6. Update $k=k+1$ and go to Step 3 .

Remark 2.18. (a) In Step 1, we would like to choose $v_{n}^{1} \in S_{L^{\perp}}$ such that it is an increasing-decreasing direction at $u_{n-1}$, i.e., $\phi(t)=J\left(u_{n-1}+t v_{n}^{1}\right)$ increases on $[0, \bar{t}]$ and decreases on $[\bar{t}, \hat{t}]$. Then, a local maximum point $\bar{t}$ of $\phi(t)$ can be used to construct $\left(t_{0}, t_{1}, \ldots, t_{n-2}, t_{n-1}\right)=(\bar{t}, 0, \ldots, 0,1)$ as an initial point to calculate $u_{n}^{1}$.

(b) In Step 5, we usually set $0<\lambda<1$ to prevent the stepsize from being too large to lose search stability.

(c) Suppose that $u=0$ is a found critical point (usually a minimum point) of $J$. Then, set $L=\{0\}$ and use the above algorithm to find a new critical point. Denote $u_{1}$ as a new critical point. Then, set $L=\left[0, u_{1}\right]=\left[u_{1}\right]$ and carry out the above algorithm to calculate a new critical point, $u_{2}$. Thus, set $L=\left[u_{1}, u_{2}\right]$ and implement the above algorithm to compute a new critical point, $u_{3}$, and so on. That is the usual way to use this minimax algorithm. If $u_{0} \not \equiv 0$ is a found critical point (usually a minimum point) of $J$, then, $I(u)=J\left(u+u_{0}\right)$ has critical point $u=0$. The above algorithm will be implemented to $I$ to find critical points of $J$.

(d) In Step 5 , from $m=1$, we solve an $n$-dimensional optimization problem to get $p\left(v_{n}^{k}(s)\right)$ and check if

$$
J\left(p\left(v_{n}^{k}(s)\right)\right)-J\left(p\left(v_{n}^{k}\right)\right) \leq-\frac{1}{4}\left|t_{0}^{k}\right| s\left\|z_{n}^{k}\right\|
$$

for $s=\frac{\lambda}{2^{m}}, m \in N$, one by one. As soon as we get $m_{n}^{k} \in N$ such that (2.7) holds for $s=\frac{\lambda}{2^{m_{n}^{k}}}$, we set $s_{n}^{k}=\frac{\lambda}{2^{m_{n}^{k}}}, v_{n}^{k+1}=v_{n}^{k}\left(s_{n}^{k}\right)$ and $u_{n}^{k+1}=p\left(v_{n}^{k+1}\right)$. When $n$ becomes larger, generally we have to do more calculation to solve the $n$-dimensional optimization problem for $p\left(v_{n}^{k}(s)\right)$.

(e) Numerical experiments show that generally the iteration number in $k$ will grow with $n$. 


\section{Convergence}

Before establishing convergence for the minimax algorithm, we generalize it to an $L-\perp$ algorithm. First, $L-\perp$ selection should be defined to a locally Lipschitz continuous functional $J$ and a closed subspace $L$ in Hilbert space $H$.

Definition 3.1. A set-valued mapping $P: S_{L^{\perp}} \rightarrow 2^{H}$ is the $L$ - $\perp$ mapping of a locally Lipschitz continuous functional $J$ w.r.t. $L$ if $\forall v \in S_{L^{\perp}}, P(v)=\{u \in[L, v]$ : $\left.\partial J(u) \cap[L, v]^{\perp} \neq \emptyset\right\}$. A single-valued mapping $p: S_{L^{\perp}} \rightarrow H$ is an $L-\perp$ selection of the locally Lipschitz continuous functional $J$ w.r.t. $L$ if $p(v) \in P(v), \forall v \in S_{L^{\perp}}$. For a given $v \in S_{L^{\perp}}$, if $p$ is locally defined in a neighborhood of $v$, we say that $J$ has a local $L-\perp$ selection $p$ at $v$.

Remark 3.2. If $L \subset H$ is a finite dimensional subspace, by Lemma 2.2, it is clear that a peak selection w.r.t. $L$ is an $L$ - $\perp$ selection w.r.t. $L$.

Then, it is easy to check that Lemma 2.14 and Theorem 2.16 are also correct to an $L$ - $\perp$ selection $p$. By this generalization, an $L-\perp$ algorithm for capturing saddle critical points of a upper semi-differentiable locally Lipschitz continuous functional $J$ can be designed. Assume that $u_{1}, u_{2}, \ldots, u_{n-1}$ are found critical points, $L=\left[u_{1}, u_{2}, \ldots, u_{n-1}, \lambda>0\right.$ is a constant and $\varepsilon$ is a small positive number. The flow chart for the algorithm is given as follows.

Step 1. Choose $v_{n}^{1} \in S_{L^{\perp}}$.

Step 2. Set $k=1$ and find $u_{n}^{k}=p\left(v_{n}^{k}\right)=t_{0}^{k} v_{n}^{k}+t_{1}^{k} u_{1}+\cdots+t_{n-1}^{k} u_{n-1}$ such that

$$
\partial J\left(u_{n}^{k}\right) \cap\left[L, v_{n}^{k}\right]^{\perp} \neq \emptyset .
$$

Step 3. Find a descent direction $w_{n}^{k}=-\operatorname{sign}\left(t_{0}^{k}\right) \frac{z_{n}^{k}}{\left\|z_{n}^{k}\right\|}$ at $u_{n}^{k}$, where $z_{n}^{k} \in \partial J\left(u_{n}^{k}\right) \cap$ $\left[L, v_{n}^{k}\right]^{\perp}$.

Step 4. If $\left\|z_{n}^{k}\right\|<\varepsilon$, then output $u_{n}^{k}$, stop. Otherwise, do Step 5.

Step 5. For each $s>0$, let $v_{n}^{k}(s)=\frac{v_{n}^{k}+s w_{n}^{k}}{\left\|v_{n}^{k}+s w_{n}^{k}\right\|}$ and find $p\left(v_{n}^{k}(s)\right)=t_{0}(s) v_{n}^{k}(s)+$ $\sum_{i=1}^{n-1} t_{i}(s) u_{i}$ such that

$$
\partial J\left(p\left(v_{n}^{k}(s)\right)\right) \cap\left[L, v_{n}^{k}(s)\right]^{\perp} \neq \emptyset,
$$

then set $v_{n}^{k+1}=v_{n}^{k}\left(s_{n}^{k}\right)$ and $u_{n}^{k+1}=p\left(v_{n}^{k+1}\right)=t_{0}^{k+1} v_{n}^{k+1}+t_{1}^{k+1} u_{1}+\cdots+$ $t_{n-1}^{k+1} u_{n-1}$, where $s_{n}^{k}$ satisfies

$$
s_{n}^{k}=\max \left\{s=\frac{\lambda}{2^{m}}\left|m \in \mathbb{N}, J\left(p\left(v_{n}^{k}(s)\right)\right)-J\left(p\left(v_{n}^{k}\right)\right) \leq-\frac{1}{4}\right| t_{0}^{k}\left|s \| z_{n}^{k}\right| \mid\right\} .
$$

Step 6. Update $k=k+1$ and go to Step 3 .

To discuss convergence of the $L-\perp$ algorithm, we need the following nonsmooth version of PS condition [5] and SC-condition in Banach space $B$.

Definition 3.3. A locally Lipschitz continuous functional $J: B \rightarrow \mathbb{R}$ satisfies the PS condition if any $\left\{u_{n}\right\} \subset B$ such that $\left\{J\left(u_{n}\right)\right\}$ is bounded and $\zeta_{n} \rightarrow 0$, where $\zeta_{n} \in \partial J\left(u_{n}\right)$ and $\left\|\zeta_{n}\right\|=\min \left\{\|\zeta\| \zeta \in \partial J\left(u_{n}\right)\right\}$, possesses a convergent subsequence.

Definition 3.4. A locally Lipschitz continuous functional $J: B \rightarrow \mathbb{R}$ satisfies the SC-condition if any $\left\{\zeta_{n} \mid \zeta_{n} \in \partial J\left(u_{n}\right)\right\}$, where $u_{n} \rightarrow u$, possesses a subsequence $\left\{\zeta_{n_{i}}\right\}$ such that $\zeta_{n_{i}} \rightarrow \zeta \in \partial J(u)$. 
Remark 3.5. Since the algorithms are for upper semi-differentiable locally Lipschitz continuous functional $J$, generalized gradient is used instead of gradient. Then, the continuity of gradient to functional $J \in C^{1}(B, \mathbb{R})$ is lost. The SC-condition is defined instead for generalized gradient of locally Lipschitz continuous functional. It may be considered as a "subsequence-continuity-condition". The SC-condition is crucial to establish subsequence and sequence convergence for the algorithm. On the other hand, for functional $J \in C^{1}(B, \mathbb{R})$, the SC-condition is automatically satisfied.

For the SC-condition, the following lemma can be verified.

Lemma 3.6. If $\Omega \subset \mathbb{R}^{n}$ is an open bounded domain, $f(x, t)$ is a measurable function on $\Omega \times \mathbb{R}^{n}$ and $f(x, t)$ satisfies

$$
|f(x, t)| \leq C_{1}+C_{2}|t|^{\sigma},
$$

for $x \in \Omega$ and $t \in \mathbb{R}$, where $0<\sigma<\frac{n+2}{n-2}$ for $n \geq 3, \sigma>0$ for $n=1,2$ and $C_{1}, C_{2}>0$ are two constants, then the locally Lipschitz continuous functional $G(u)=\int_{\Omega} \int_{0}^{u(x)} f(x, s) d s d x$ satisfies the SC-condition in $H_{0}^{1}(\Omega)$.

Proof. First, according to Chang [5, $G(u)=\int_{\Omega} \int_{0}^{u(x)} f(x, s) d s d x$ is locally Lipschitz continuous in $L^{\sigma+1}(\Omega)$ and $H_{0}^{1}(\Omega)$ as well. Suppose that $u_{n} \rightarrow u$ in $H_{0}^{1}(\Omega)$. By the Sobolev embedding theorem, $u_{n} \rightarrow u$ in $L^{\sigma+1}(\Omega)$. By Theorem 2.2 in $[5]$ and (b) in Proposition 1.7. $\left\{\zeta_{n} \mid \zeta_{n} \in \partial J\left(u_{n}\right)\right\}$ is bounded in $L^{\eta}(\Omega)$, where $\eta=\frac{\sigma+1}{\sigma}$. Since $L^{\eta}(\Omega)$ is reflexive, $\left\{\zeta_{n}\right\}$ has a subsequence which is weakly convergent to $\zeta \in \partial J(u)$. We assume that $\zeta_{n} \rightarrow \zeta$ weakly in $L^{\eta}(\Omega)$. Then, $\left\{\zeta_{n}\right\}$ must have a subsequence which is convergent to $\zeta$ in $H^{-1}(\Omega)$. Otherwise, there is $\epsilon>0$ such that $\left\|\zeta_{n}-\zeta\right\|_{H^{-1}(\Omega)} \geq \epsilon$. This means there is $\left\{\xi_{n}\right\}$, where $\xi_{n} \in H_{0}^{1}(\Omega)$ and $\left\|\xi_{n}\right\|_{H_{0}^{1}(\Omega)}=1$, and $\rho>0$ such that

$$
\int_{\Omega}\left(\zeta_{n}(x)-\zeta(x)\right) \xi_{n}(x) d x \geq \rho .
$$

By the Rellich-Kondrachov theorem (cf. [1]), $\left\{\xi_{n}\right\}$ has a subsequence which is convergent to $\xi$ in $L^{\sigma+1}(\Omega)$. We can assume $\xi_{n} \rightarrow \xi$. Then,

$$
\begin{aligned}
& \left|\int_{\Omega}\left(\zeta_{n}(x)-\zeta(x)\right) \xi_{n}(x) d x\right| \\
\leq & \int_{\Omega}\left|\left(\zeta_{n}(x)-\zeta(x)\right)\left(\xi_{n}(x)-\xi(x)\right)\right| d x+\left|\int_{\Omega}\left(\zeta_{n}(x)-\zeta(x)\right) \xi(x) d x\right| \\
\leq & \left\|\zeta_{n}-\zeta\right\|_{L^{\eta}(\Omega)}\left\|\xi_{n}-\xi\right\|_{L^{\sigma+1}(\Omega)}+\left|\int_{\Omega}\left(\zeta_{n}(x)-\zeta(x)\right) \xi(x) d x\right| \\
\leq & \left(\left\|\zeta_{n}\right\|_{L^{\eta}(\Omega)}+\|\zeta\|_{L^{\eta}(\Omega)}\right)\left\|\xi_{n}-\xi\right\|_{L^{\sigma+1}(\Omega)}+\left|\int_{\Omega}\left(\zeta_{n}(x)-\zeta(x)\right) \xi(x) d x\right|,
\end{aligned}
$$

where $\eta=\frac{\sigma+1}{\sigma}$, means

$$
\int_{\Omega}\left(\zeta_{n}(x)-\zeta(x)\right) \xi_{n}(x) d x \rightarrow 0
$$

This is a contradiction to (3.1). Thus, $\left\{\zeta_{n}\right\}$ has a subsequence which is convergent to $\zeta$ in $H^{-1}(\Omega)$. Hence, by (f) in Proposition 1.7, the locally Lipschitz continuous functional $G(u)=\int_{\Omega} \int_{0}^{u(x)} f(x, s) d s d x$ satisfies the SC-condition in $H_{0}^{1}(\Omega)$. 
Remark 3.7. A similar result can be established in Banach space $W_{0}^{1, q}(\Omega), q>1$.

To establish subsequence convergence, the following lemma is crucial.

Lemma 3.8. Let $H$ be a Hilbert space with $H=L \oplus L^{\perp}$ for a finite dimensional subspace $L \subset H$. Assume that $J$ is a upper semi-differentiable locally Lipschitz continuous functional and $p$ is a local $L-\perp$ selection of $J$ w.r.t. $L$ around $v \in$ $S_{L^{\perp}}$ such that $p(v)$ is not a critical point, $p$ has super-linear property at $v$ and $d(p(v), L)>0$. If $v_{j} \rightarrow v$ and $z_{j} \rightarrow z$, where $v_{j} \in S_{L^{\perp}}, z_{j} \in \partial J\left(p\left(v_{j}\right)\right) \cap\left[L, v_{j}\right]^{\perp}$ and $z \in \partial J(p(v)) \cap[L, v]^{\perp}$, then there is $s_{0}=\frac{\lambda}{2^{m_{0}}}, m_{0} \in \mathbb{N}$ such that, as $j$ is large,

$$
J\left(p\left(v_{j}\left(s_{0}\right)\right)\right)-J\left(p\left(v_{j}\right)\right)<-\frac{1}{4} s_{0}\left|t_{j}\right|\left\|z_{j}\right\|,
$$

where $v_{j}\left(s_{0}\right)=\frac{v_{j}+s_{0} w_{j}}{\left\|v_{j}+s_{0} w_{j}\right\|}, w_{j}=-\operatorname{sign}\left(t_{j}\right) \frac{z_{j}}{\left\|z_{j}\right\|}, p\left(v_{j}\right)=t_{j} v_{j}+u_{j}, u_{j} \in L$.

Proof. By Lemma 2.14, as $s>0$ is small,

$$
J(p(v(s)))-J(p(v))<-\frac{1}{4} s\left|t_{v}\right|\|z\|,
$$

where $v(s)=\frac{v+s w}{\|v+s w\|}, w=-\operatorname{sign}\left(t_{v}\right) \frac{z}{\|z\|}$ and $p(v)=t_{v} v+u, u \in L$. Thus, since $p$ is continuous at $v, v_{j} \rightarrow v$ and $z_{j} \rightarrow z$, there is $s_{0}=\frac{\lambda}{2^{m_{0}}}, m_{0} \in \mathbb{N}$ such that, as $j$ is large,

$$
J\left(p\left(v_{j}\left(s_{0}\right)\right)\right)-J\left(p\left(v_{j}\right)\right)<-\frac{1}{4} s_{0}\left|t_{j}\right|\left\|z_{j}\right\|,
$$

where $v_{j}\left(s_{0}\right)=\frac{v_{j}+s_{0} w_{j}}{\left\|v_{j}+s_{0} w_{j}\right\|}, w_{j}=-\operatorname{sign}\left(t_{j}\right) \frac{z_{j}}{\left\|z_{j}\right\|}, p\left(v_{j}\right)=t_{j} v_{j}+u_{j}, u_{j} \in L$.

First, we prove a subsequence convergence result for the $L$ - $\perp$ algorithm.

Theorem 3.9. If an upper semi-differentiable locally Lipschitz continuous functional $J$ satisfies the $P S$ condition and the $S C$-condition, $\lambda \in(0,1),\left\{v_{n}^{k}\right\}$ is a sequence generated by the $L-\perp$ algorithm and the $L-\perp$ selection $p$ of $J$ satisfies:

(a) $p$ has super-linear property on $S_{L^{\perp}}$,

(b) $d\left(p\left(v_{n}^{k}\right), L\right) \geq \alpha>0, \forall k=1,2, \ldots$, and

(c) $\inf _{1 \leq k<\infty} J\left(p\left(v_{n}^{k}\right)\right)>-\infty$,

(d) $\left\{v_{n}^{k}\right\}$ has a subsequence $\left\{v_{n}^{k_{i}}\right\}$ such that $u_{n}^{k_{i}}=p\left(v_{n}^{k_{i}}\right)$ converges to a critical point of $J$,

(e) if a subsequence $v_{n}^{k_{i}} \rightarrow v_{0}$ as $i \rightarrow \infty$, then $u_{0}=p\left(v_{0}\right)$ is a critical point of $J$.

Proof. (a) By the step-size rule, we have

$$
J\left(u_{n}^{k+1}\right)-J\left(u_{n}^{k}\right) \leq-\frac{1}{4}\left|t_{0}^{k}\right| s_{n}^{k}\left\|z_{n}^{k}\right\|,
$$

for $k=1,2, \ldots$. On the other hand, by Lemma 2.4 .

$$
\begin{aligned}
\left\|v_{n}^{k+1}-v_{n}^{k}\right\| & =\left\|\frac{v_{n}^{k}+s_{n}^{k} w_{n}^{k}}{\left\|v_{n}^{k}+s_{n}^{k} w_{n}^{k}\right\|}-v_{n}^{k}\right\| \\
\leq \quad & \frac{2\left\|s_{n}^{k} w_{n}^{k}\right\|}{\left\|v_{n}^{k}+s_{n}^{k} w_{n}^{k}\right\|} \leq \frac{2 s_{n}^{k}}{\left|1-s_{n}^{k}\right|} \leq \frac{2 s_{n}^{k}}{1-\lambda}
\end{aligned}
$$

and, by assumption (b),

$$
\left|t_{0}^{k}\right| \geq d\left(p\left(v_{n}^{k}\right), L\right) \geq \alpha
$$


Then,

$$
J\left(u_{n}^{k+1}\right)-J\left(u_{n}^{k}\right) \leq-\frac{1}{4}\left|t_{0}^{k}\right| s_{n}^{k}\left\|z_{n}^{k}\right\| \leq-\frac{(1-\lambda)}{8} \alpha\left\|v_{n}^{k+1}-v_{n}^{k}\right\|\left\|z_{n}^{k}\right\| .
$$

Thus, there is a subsequence $z_{n}^{k_{i}} \rightarrow 0$. Otherwise, there is $\delta>0$ such that $\left\|z_{n}^{k}\right\| \geq \delta$ for any $k$. From (3.4), we have

$$
J\left(u_{n}^{k+1}\right)-J\left(u_{n}^{k}\right) \leq-\frac{(1-\lambda)}{8} \alpha \delta\left\|v_{n}^{k+1}-v_{n}^{k}\right\|, \quad \forall k=1,2, \ldots
$$

Adding up two sides of (3.5) gives

$$
\lim _{k \rightarrow \infty} J\left(u_{n}^{k}\right)-J\left(u_{n}^{1}\right)=\sum_{k=1}^{\infty}\left[J\left(u_{n}^{k+1}\right)-J\left(u_{n}^{k}\right)\right] \leq-\frac{(1-\lambda)}{8} \alpha \delta \sum_{k=0}^{\infty}\left\|v_{n}^{k+1}-v_{n}^{k}\right\|,
$$

i.e., $\left\{v_{n}^{k}\right\}$ is a Cauchy sequence. Thus $v_{n}^{k} \rightarrow \hat{v} \in S_{L^{\perp}}$. Since $J$ satisfies the SCcondition and $p$ is continuous, there is a subsequence $z_{n}^{k_{i}} \rightarrow z \in \partial J(p(\hat{v})) \cap[L, \hat{v}]^{\perp}$ with $z \neq 0$. On the other hand, by (3.3) and adding up two sides of (3.4), we have

$$
\lim _{k \rightarrow \infty} J\left(u_{n}^{k}\right)-J\left(u_{n}^{1}\right) \leq-\frac{1}{4} \sum_{k=1}^{\infty}\left|t_{0}^{k}\right| s_{n}^{k}\left\|z_{n}^{k}\right\| \leq-\frac{1}{4} \alpha \delta \sum_{k=0}^{\infty} s_{n}^{k},
$$

or $s_{n}^{k} \rightarrow 0$ as $k \rightarrow \infty$. It contradicts Lemma 3.8. When there is a subsequence $z_{n}^{k_{i}} \rightarrow 0$, by the PS condition, we can assume $u_{n}^{k_{i}}=p\left(v_{n}^{k_{i}}\right) \rightarrow u_{0}$. By (f) in Proposition 1.7. $0 \in \partial J\left(u_{0}\right)$, i.e., $u_{0}$ is a critical point.

(b) Suppose $u_{0}=p\left(v_{0}\right)$ is not a critical point. Then, there is $\delta>0$ such that $\left\|z_{n}^{k_{i}}\right\| \geq \delta, i=1,2, \ldots$ By (3.2) and (3.3), we have

$$
J\left(u_{n}^{k_{i}+1}\right)-J\left(u_{n}^{k_{i}}\right) \leq-\frac{1}{4}\left|t_{0}^{k_{i}}\right| s_{n}^{k_{i}}\left\|z_{n}^{k_{i}}\right\|<-\frac{1}{4} \alpha \delta s_{n}^{k_{i}} .
$$

Since $\sum_{k=1}^{\infty}\left[J\left(u_{n}^{k+1}\right)-J\left(u_{n}^{k}\right)\right]=\lim _{k \rightarrow \infty} J\left(u_{n}^{k}\right)-J\left(u_{n}^{1}\right)$, it leads to $\lim _{i \rightarrow \infty}\left(J\left(u_{n}^{k_{i}+1}\right)-\right.$ $\left.J\left(u_{n}^{k_{i}}\right)\right)=0$. Hence, $\lim _{i \rightarrow \infty} s_{n}^{k_{i}}=0$. This contradicts Lemma 3.8. Thus, $u_{0}$ is a critical point.

Remark 3.10. Comparing this theorem with Theorem 3.2 in [11, we assume superlinear property of $p$ on $S_{L^{\perp}}$ in this theorem instead of continuity of $p$ on $S_{L^{\perp}}$ in Theorem 3.2 in 11]. By Lemma 2.13, if $J \in C^{1}(H, \mathbb{R})$, these two assumptions are equivalent.

To give a sequence convergence, the following Ekeland's variational principle 12 will be used first to prove an abstract existence-convergence result that is actually independent of the algorithm.

Lemma 3.11 (Ekeland's variational principle). Let $X$ be a complete metric space and $J: X \rightarrow \mathbb{R} \cup\{+\infty\}$ be an upper semicontinuous functional bounded from below. Then, for any $\epsilon>0$ and $x_{0} \in X$ with $J\left(x_{0}\right)<+\infty$, there is $\bar{x} \in X$ such that

$$
J(\bar{x})+\epsilon d\left(x_{0}, \bar{x}\right) \leq J\left(x_{0}\right) \text { and } J(x)+\epsilon d(x, \bar{x})>J(\bar{x}) \quad \forall x \in X \text { and } x \neq \bar{x} .
$$

Then, sequence convergence results are presented as Corollary 3.14 and Theorem 3.17 by this abstract existence-convergence result. Denote $K=\{u \in B \mid 0 \in \partial J(u)\}$ and $K_{c}=\{u \in B \mid 0 \in \partial J(u), J(u)=c\}$, where $J$ is a locally Lipschitz continuous functional in Banach space $B$. If $J$ satisfies the PS condition, $K_{c}$ is compact. 
Theorem 3.12. Let $H$ be a Hilbert space with $H=L \oplus L^{\perp}$ for a finite dimensional subspace $L \subset H$ and $U=V \cap S_{L^{\perp}} \neq \emptyset$, where $V \subset H$ is open. Assume that an upper semi-differentiable locally Lipschitz continuous functional $J$ satisfies the PS condition and an $L-\perp$ selection $p$ of $J$ satisfies:

(a) $p$ has super-linear property in $U$ and $p$ is continuous on $\bar{U}$, where $\bar{U}$ is the closure of $U$ on $S_{L^{\perp}}$,

(b) $\inf _{v \in U} d(p(v), L) \geq \alpha>0$, and

(c) $\inf _{v \in \partial \bar{U}} J(p(v))>c=\inf _{v \in U} J(p(v))>-\infty$, where $\partial \bar{U}$ is the boundary of $\bar{U}$ on $S_{L^{\perp}}$.

Then, $K_{c}^{p}=p(U) \cap K_{c} \neq \emptyset$, where $p(U)=\{p(u) \mid u \in U\}$ and for any $\left\{v^{k}\right\} \subset U$ with $J\left(u^{k}\right) \rightarrow c$, where $u^{k}=p\left(v^{k}\right)$, we have

$$
\lim _{k \rightarrow \infty} d\left(u^{k}, K_{c}^{p}\right)=0 .
$$

Proof. Define

$$
\hat{J}(p(v))= \begin{cases}J(p(v)) & v \in \bar{U} \\ +\infty & v \notin \bar{U}\end{cases}
$$

Then, $\hat{J}(p(\cdot))$ is upper semicontinuous and bounded from below on the complete metric space $S_{L^{\perp}}$. By assumption (c), either there is $v \in U$ such that $J(p(v))=c$ or there is a sequence $\left\{v^{k}\right\} \subset U$ such that $J\left(p\left(v^{k}\right)\right)>c$ and $J\left(p\left(v^{k}\right)\right) \rightarrow c$. For the first case, $p(v) \in K_{c}^{p}=p(U) \cap K_{c}$ by Theorem 2.16, Of course, $K_{c}^{p} \neq \emptyset$. For the second case, denote $u^{k}=p\left(v^{k}\right)$. Applying Ekeland's variational principle to $\hat{J}(p(\cdot))$, for every $v^{k} \in U$ and $\delta^{k}=\left(J\left(u^{k}\right)-c\right)^{\frac{1}{2}}$, there is $\bar{v}^{k} \in S_{L^{\perp}}$ such that

$$
\begin{aligned}
\hat{J}\left(p\left(\bar{v}^{k}\right)\right)-\hat{J}(p(v)) & <\delta^{k}\left\|\bar{v}^{k}-v\right\| \quad \forall v \in S_{L^{\perp}}, \\
\hat{J}\left(p\left(\bar{v}^{k}\right)\right)-\hat{J}\left(p\left(v^{k}\right)\right) & \leq-\delta^{k}\left\|\bar{v}^{k}-v^{k}\right\| .
\end{aligned}
$$

By (3.8), the definition of $\hat{J}(p(\cdot))$ and $v^{k} \in U$, we have $\bar{v}^{k} \in \bar{U}$ and then, from (3.7) and (3.8),

$$
\begin{aligned}
J\left(p\left(\bar{v}^{k}\right)\right)-J(p(v)) & <\delta^{k}\left\|\bar{v}^{k}-v\right\| \quad \forall v \in U, \\
J\left(p\left(\bar{v}^{k}\right)\right)-J\left(p\left(v^{k}\right)\right) & \leq-\delta^{k}\left\|\bar{v}^{k}-v^{k}\right\| .
\end{aligned}
$$

It follows

$$
c \leq J\left(p\left(\bar{v}^{k}\right)\right) \leq J\left(u^{k}\right)-\delta^{k}\left\|\bar{v}^{k}-v^{k}\right\|
$$

i.e,

$$
\left\|\bar{v}^{k}-v^{k}\right\| \leq \delta^{k}
$$

Then, by (3.11) and (3.12), $J\left(u^{k}\right)=J\left(p\left(v^{k}\right)\right) \rightarrow c$ implies $J\left(p\left(\bar{v}^{k}\right)\right) \rightarrow c$. Thus, by assumptions (b) and (c), we have $\bar{v}^{k} \in U$ and

$$
d\left(p\left(\bar{v}^{k}\right), L\right) \geq \alpha>0
$$

for large $k$. For these large $k$, if there is nonzero $\bar{z}^{k} \in \partial J\left(p\left(\bar{v}^{k}\right)\right) \cap\left[L, \bar{v}^{k}\right]^{\perp}$, then, when $s$ is small, by Lemma 2.14,

$$
J\left(p\left(\bar{v}^{k}(s)\right)\right)-J\left(p\left(\bar{v}^{k}\right)\right)<-\frac{s}{4}\left|\bar{t}^{k}\right|\left\|\bar{z}^{k}\right\|,
$$


where $\bar{v}^{k}(s)=\frac{\bar{v}^{k}+s w^{k}}{\left\|\bar{v}^{k}+s w^{k}\right\|} \in U, w^{k}=-\operatorname{sign}\left(\bar{t}^{k}\right) \frac{\bar{z}^{k}}{\left\|\bar{z}^{k}\right\|}, p\left(\bar{v}^{k}\right)=\bar{t}^{k} \bar{v}^{k}+\bar{w}^{k}$ and $\bar{w}^{k} \in L$ and by Lemma 2.4,

$$
\left\|v^{k}(s)-v^{k}\right\|=\left\|\frac{v^{k}+s w^{k}}{\left\|v^{k}+s w^{k}\right\|}-v^{k}\right\| \leq \frac{2\left\|s w^{k}\right\|}{\left\|v^{k}+s w^{k}\right\|} \leq \frac{2 s}{1-s} \leq 4 s,
$$

i.e.,

$$
J\left(p\left(\bar{v}^{k}(s)\right)\right)-J\left(p\left(\bar{v}^{k}\right)\right)<-\frac{1}{16} \alpha\left\|\bar{z}^{k}\right\|\left\|\bar{v}^{k}(s)-\bar{v}^{k}\right\|,
$$

by (3.13). Hence, by (3.9), we have

$$
\left\|\bar{z}^{k}\right\|<\frac{16}{\alpha} \delta^{k}
$$

which implies $\bar{z}^{k} \rightarrow 0$. Then, by the PS condition, $\left\{p\left(\bar{v}^{k}\right)\right\}$ has a convergent subsequence $\left\{p\left(\bar{v}^{k_{j}}\right)\right\}$. Since $\left\{p\left(\bar{v}^{k_{j}}\right)\right\}$ is convergent, $\bar{v}^{k_{j}} \in S_{L^{\perp}}$ and $\bar{w}^{k_{j}} \in L,\left\{t^{k_{j}} \bar{v}^{k_{j}}\right\}$ is convergent, i.e., we can assume $\left\{t^{k_{j}}\right\}$ and $\left\{\bar{v}^{k_{j}}\right\}$ are both convergent by (3.13). Denote $\bar{v}=\lim _{j \rightarrow \infty} \bar{v}^{k_{j}}$. By (f) in Proposition 1.7 and (3.14), $0 \in \partial J(p(\bar{v}))$, i.e., $p(\bar{v})$ is a critical point and by (3.12), $\bar{v}=\lim _{j \rightarrow \infty} v^{k_{j}}$. By assumption (a),

$$
p(\bar{v})=\lim _{j \rightarrow \infty} p\left(v^{k_{j}}\right) \text { and } J(p(\bar{v}))=c
$$

and then, by assumption (c), it is clear that

$$
\bar{v} \in U \text { and } \bar{u}=p(\bar{v}) \in K_{c}^{p} \neq \emptyset .
$$

For any $\left\{v^{k}\right\} \subset U$ with $J\left(u^{k}\right) \rightarrow c$, where $u^{k}=p\left(v^{k}\right)$, let $\beta$ be any limit point of $\left\{d\left(u^{k}, K_{c}^{p}\right)\right\}$ and $u^{k_{j}}=p\left(v^{k_{j}}\right) \in\left\{u^{k}\right\}$ such that $\lim _{j \rightarrow \infty} d\left(u^{k_{j}}, K_{c}^{p}\right)=\beta$. If there is a subsequence $\left\{u^{k_{j_{i}}}\right\} \subseteq\left\{u^{k_{j}}\right\}$ such that $J\left(u^{k_{j_{i}}}\right)=c$, then, by Theorem 2.16. $u^{k_{j_{i}} \in K_{c}^{p}}$, i.e., $\beta=0$. Otherwise, we can assume $J\left(u^{k_{j}}\right)>c, j=1,2, \ldots$. Consider $\left\{u^{k_{j}}\right\}$ as $\left\{u^{k}\right\}$. To $\left\{u^{k_{j}}\right\}$, by using above argument for $\left\{u^{k}\right\}$, we know there is a subsequence $\left\{u^{k_{j_{i}}}\right\} \subseteq\left\{u^{k_{j}}\right\}$ such that $\bar{u}=\lim _{i \rightarrow \infty} p\left(v_{k_{j_{i}}}\right) \in K_{c}^{p}$, i.e., $\beta=0$. Thus, every limit point of $\left\{d\left(u^{k}, K_{c}^{p}\right)\right\}$ is zero, i.e.,

$$
\lim _{k \rightarrow \infty} d\left(u^{k}, K_{c}^{p}\right)=0 .
$$

Remark 3.13. Comparing this theorem with Theorem 3.5 in [15, we assume superlinear property of $p$ in $U$ and continuity of $p$ on $\bar{U}$, where $\bar{U}$ is the closure of $U$ on $S_{L^{\perp}}$ in this theorem instead of continuity of $p$ on $\bar{U}$, where $\bar{U}$ is the closure of $U$ on $S_{L^{\perp}}$ in Theorem 3.5 in [15]. By Lemma 2.13, if $J \in C^{1}(H, \mathbb{R})$, these two assumptions are equivalent.

Corollary 3.14. Let $H$ be a Hilbert space with $H=L \oplus L^{\perp}$ for a finite dimensional subspace $L \subset H$ and $V_{1}, V_{2} \subset H$ two open sets with $\emptyset \neq U_{2} \equiv V_{2} \cap S_{L^{\perp}} \subset V_{1} \cap S_{L^{\perp}} \equiv$ $U_{1}$. Assume that a upper semi-differentiable locally Lipschitz continuous functional $J$ satisfies the PS condition and an $L-\perp$ selection $p$ of $J$ satisfies:

(a) $p$ has super-linear property in $U_{1}$,

(b) $\inf _{v \in U_{1}} d(p(v), L) \geq \alpha>0$ and $c=\inf _{v \in U_{1}} J(p(v))>-\infty$,

(c) there is $d>0$ with

$$
\inf \left\{J(p(v)) \mid v \in U_{1}, d\left(v, \partial U_{1}\right) \leq d\right\}=a>b=\sup \left\{J(p(v)) \mid v \in U_{2}\right\},
$$

where $\partial U_{1}$ is the boundary of $U_{1}$ on $S_{L^{\perp}}$,

(d) given $\left\{v_{k}\right\}$ such that $v_{1} \in U_{2},\left\|v_{k+1}-v_{k}\right\|<d, J\left(u_{k+1}\right)<J\left(u_{k}\right)$ and $\left\{u_{k}\right\}$ has a subsequence that converges to a critical point $u_{0}$, where $u_{k}=p\left(v_{k}\right)$, and

(e) $K_{c}^{p}=p\left(U_{1}\right) \cap K \subset K_{c}$, where $p\left(U_{1}\right)=\left\{p(u) \mid u \in U_{1}\right\}$. 
Then,

$$
\lim _{k \rightarrow \infty} d\left(u_{k}, K_{c}^{p}\right)=0 .
$$

Proof. First, we prove that $v_{k} \in U_{1}$ and $d\left(v_{k}, \partial U_{1}\right)>d, k=1,2, \ldots$. In fact, if $v_{k} \in U_{1}, d\left(v_{k}, \partial U_{1}\right)>d$ and $J\left(u_{k}\right) \leq b$, then, by assumption (d), $v_{k+1} \in U_{1}$ and $J\left(u_{k+1}\right)<b$, i.e., $v_{k+1} \in U_{1}$ and $d\left(v_{k+1}, \partial U_{1}\right)>d$ by assumption (c). Thus, since $v_{1} \in U_{2}$, we have $v_{k} \in U_{1}$ and $d\left(v_{k}, \partial U_{1}\right)>d$ for $k=1,2, \ldots$, by assumption (c). Since $K_{c}^{p}=p\left(U_{1}\right) \cap K \subset K_{c}$ and $\left\{u_{k}\right\}$ has a subsequence which converges to a critical point $u_{0}$, we have $u_{0} \in K_{c}^{p} \neq \emptyset$. Denote $U=\left\{v \in U_{1} \mid d\left(v, \partial U_{1}\right)>d\right\}$. Then, by the monotonicity of $\left\{J\left(u_{k}\right)\right\}$, we have $J\left(u_{k}\right) \rightarrow c=\inf _{v \in U} J(p(v))$ as $k \rightarrow \infty$, and

$$
\inf _{v \in \partial \bar{U}} J(p(v)) \geq a>b \geq J\left(p\left(v_{1}\right)\right) \geq c=\inf _{v \in U} J(p(v)),
$$

where $\partial \bar{U}$ is the boundary of $U$ on $S_{L^{\perp}}$. Thus, all assumptions of Theorem 3.12 are satisfied and the conclusion follows.

Remark 3.15. Comparing this corollary with Corollary 3.6 in [15, we assume superlinear property of $p$ in $U_{1}$ in this theorem instead of continuity of $p$ in $U_{1}$ in Corollary 3.6 in [15. By Lemma 2.13, if $J \in C^{1}(H, \mathbb{R})$, these two assumptions are equivalent.

To better understand Corollary 3.14 we give the following lemma.

Lemma 3.16. Let $H$ be a Hilbert space with $H=L \oplus L^{\perp}$ for a finite dimensional subspace $L \subset H$ and $V_{1}, V_{2} \subset H$ two open sets with $\emptyset \neq U_{2} \equiv V_{2} \cap S_{L^{\perp}} \subset V_{1} \cap S_{L^{\perp}} \equiv$ $U_{1}$. Assume that an upper semi-differentiable locally Lipschitz continuous functional $J$ satisfies the $P S$ condition and the $S C$-condition, an $L-\perp$ selection $p$ of $J$ satisfies:

(a) $p$ has super-linear property in $U_{1}$,

(b) $\inf _{v \in U_{1}} d(p(v), L) \geq \alpha>0$ and $c=\inf _{v \in U_{1}} J(p(v))>-\infty$,

(c) there is $d>0$ with

$$
\inf \left\{J(p(v)) \mid v \in U_{1}, d\left(v, \partial U_{1}\right) \leq d\right\}=a>b=\sup \left\{J(p(v)) \mid v \in U_{2}\right\},
$$

where $\partial U_{1}$ is the boundary of $U_{1}$ on $S_{L^{\perp}}$, and $\left\{v_{n}^{k}\right\}$ is a sequence generated by the $L-\perp$ algorithm according to the $L-\perp$ selection $p$ with initial point $v_{n}^{1} \in U_{2}$ and $\lambda \in\left(0, \frac{d}{d+2}\right)$. Then, $\left\|v_{n}^{k+1}-v_{n}^{k}\right\|<d, J\left(u_{n}^{k+1}\right)<J\left(u_{n}^{k}\right)$ and $\left\{u_{n}^{k}\right\}$ has a subsequence that converges to a critical point $u_{n}^{0}$, where $u_{n}^{k}=p\left(v_{n}^{k}\right)$.

Proof. First, by Lemma 2.4, we have

$$
\left\|v_{n}^{k+1}-v_{n}^{k}\right\|=\left\|\frac{v_{n}^{k}+s_{n}^{k} w_{n}^{k}}{\left\|v_{n}^{k}+s_{n}^{k} w_{n}^{k}\right\|}-v_{n}^{k}\right\| \leq \frac{2\left\|s_{n}^{k} w_{n}^{k}\right\|}{\left\|v_{n}^{k}+s_{n}^{k} w_{n}^{k}\right\|} \leq \frac{2 s_{n}^{k}}{\left|1-s_{n}^{k}\right|} \leq \frac{2 \lambda}{1-\lambda} .
$$

Then, $\lambda \in\left(0, \frac{d}{d+2}\right)$ guarantees

$$
\left\|v_{n}^{k+1}-v_{n}^{k}\right\|<d .
$$

Since the $L-\perp$ algorithm is a descent method, we have

$$
J\left(u_{n}^{k+1}\right)<J\left(u_{n}^{k}\right) .
$$

Consider $\left\{v_{n}^{k}\right\}$ as $\left\{v_{k}\right\}$. By exactly mimicking the verification of Corollary 3.14 to $\left\{v_{k}\right\}$, we can show that

$$
\left\{v_{n}^{k}\right\} \subset U=\left\{v \in U_{1} \mid d\left(v, \partial U_{1}\right)>d\right\},
$$

where $\partial U_{1}$ is the boundary $U_{1}$ on $S_{L^{\perp}}$. Thus,

$$
\overline{\left\{v_{n}^{k}\right\}} \subset U_{1} \text {, }
$$


where $\overline{\left\{v_{n}^{k}\right\}}$ is the closure of $\left\{v_{n}^{k}\right\}$ on $S_{L^{\perp}}$. Then, by exactly mimicking the verification of Theorem 3.9, we can prove that $\left\{u_{n}^{k}\right\}$ has a subsequence that converges to a critical point $u_{n}^{0}$.

By Corollary 3.14 and Lemma 3.16, Theorem 3.17 immediately follows.

Theorem 3.17. Let $H$ be a Hilbert space with $H=L \oplus L^{\perp}$ for a finite dimensional subspace $L \subset H$ and $V_{1}, V_{2} \subset H$ two open sets with $\emptyset \neq U_{2} \equiv V_{2} \cap S_{L^{\perp}} \subset V_{1} \cap S_{L^{\perp}} \equiv$ $U_{1}$. Assume that an upper semi-differentiable locally Lipschitz continuous functional $J$ satisfies the $P S$ condition and the $S C$-condition, an $L-\perp$ selection $p$ of $J$ satisfies

(a) $p$ has super-linear property in $U_{1}$,

(b) $\inf _{v \in U_{1}} d(p(v), L) \geq \alpha>0$ and $c=\inf _{v \in U_{1}} J(p(v))>-\infty$,

(c) there is $d>0$ with

$$
\inf \left\{J(p(v)) \mid v \in U_{1}, d\left(v, \partial U_{1}\right) \leq d\right\}=a>b=\sup \left\{J(p(v)) \mid v \in U_{2}\right\},
$$

where $\partial U_{1}$ is the boundary of $U_{1}$ on $S_{L^{\perp}}$, and

(d) $K_{c}^{p}=p\left(U_{1}\right) \cap K \subset K_{c}$, where $p\left(U_{1}\right)=\left\{p(u) \mid u \in U_{1}\right\}$,

and $\left\{v_{n}^{k}\right\}$ is a sequence generated by the $L-\perp$ algorithm according to the $L-\perp$ selection $p$ with initial point $v_{n}^{1} \in U_{2}$ and $\lambda \in\left(0, \frac{d}{d+2}\right)$. Then,

$$
\lim _{k \rightarrow \infty} d\left(u_{n}^{k}, K_{c}^{p}\right)=0 .
$$

\section{Numerical EXPERIMENT RESUlts}

As we pointed out before, a typical example on application of nonsmooth critical point theory to partial differential equations is the Dirichlet problem (1.1), i.e.,

$$
\left\{\begin{array}{l}
-\Delta u(x)=f(x, u(x)), x \in \Omega \\
\left.u(x)\right|_{x \in \partial \Omega}=0
\end{array}\right.
$$

where $\Omega$ is an open bounded domain in $\mathbb{R}^{m}$ with smooth boundary $\partial \Omega, f(x, t)$ is a measurable function defined on $\Omega \times \mathbb{R}$ and for every $x \in \Omega, f(x, t)$ is locally bounded. The corresponding variational functional on $H_{0}^{1}(\Omega)$ is

$$
J(u)=\frac{1}{2} \int_{\Omega}|\nabla u(x)|^{2} d x-\int_{\Omega} F(x, u(x)) d x,
$$

where $F(x, t)=\int_{0}^{t} f(x, s) d s$. Denote $G(u)=\int_{\Omega} F(x, u(x)) d x$. By Theorem 1.3, to find a $u_{0} \in H_{0}^{1}(\Omega)$ such that

$$
-\Delta u_{0}(x) \in\left[\bar{f}\left(x, u_{0}(x)\right), \hat{f}\left(x, u_{0}(x)\right)\right]
$$

for all $x \in \Omega$, we can find a critical point of $J$ (4.1). As a numerical example, we set

$$
f(x, t)=\left\{\begin{array}{l}
c \alpha|x|^{r}|t|^{\alpha-2} t, \text { if }|t|>1, \\
c \beta|x|^{r}|t|^{\beta-2} t, \text { if }|t| \leq 1,
\end{array}\right.
$$

where $c>0, \alpha>\beta>2, \alpha<\frac{2 m}{m-2}$ for $m \geq 3, r \geq 0$ and $|x|$ is the Euclidean norm of $x \in \mathbb{R}^{m}$. If $c=1, \alpha=\beta>2$ and $r=0$ in (4.2), the Dirichlet problem (1.1) is the Lane-Emden equation and if $c=1, \alpha=\beta>2$ and $r>0$ in (4.2), the Dirichlet problem (1.1) is the Hénon equation. These two equations were used as numerical examples for finding smooth saddle critical points [6], [10], [11. If $r \geq 0$, $\alpha>\beta>2$ and $\alpha<\frac{2 m}{m-2}$ for $m \geq 3, J$ (4.1) is locally Lipschitz continuous and it is not differentiable everywhere. If $c>0, r \geq 0, \alpha>\beta>2$ and $\alpha<\frac{2 m}{m-2}$ for 
$m \geq 3$, it is easy to check that $f(x, t)$ in (4.2) satisfies all assumptions in Theorem 1.5. Thus,

$$
F(x, t)=\left\{\begin{array}{l}
c|x|^{r}|t|^{\alpha}, \text { if }|t|>1 \\
c|x|^{r}|t|^{\beta}, \text { if }|t| \leq 1
\end{array}\right.
$$

and

$$
\partial G(u)=\left\{\zeta: \Omega \rightarrow \mathbb{R} \mid \zeta \text { is measurable, } \zeta(x) \in f_{-}^{+}(x, u(x)) \forall x \in \Omega\right\},
$$

where

$$
\begin{aligned}
f_{-}^{+}(x, u(x))= & {[f(x, u(x)-0), f(x, u(x)+0)] } \\
= & \begin{cases}\left\{c \alpha|x|^{r}|u(x)|^{\alpha-2} u(x)\right\}, & \text { if }|u(x)|>1, \\
\left\{c \beta|x|^{r}|u(x)|^{\beta-2} u(x)\right\}, & \text { if }|u(x)|<1, \\
{\left[c \beta|x|^{r}, c \alpha|x|^{r}\right],} & \text { if } u(x)=1, \\
{\left[-c \alpha|x|^{r},-c \beta|x|^{r}\right],} & \text { if } u(x)=-1 .\end{cases}
\end{aligned}
$$

$f(x, t)$ in (4.2) with $\alpha=6, \beta=4, r=0$ and $c=1$ is an example in [13.

Before we discuss the details on numerical experiment, we establish several lemmas for $J$ (4.1).

Lemma 4.1. Assume that $L=\{0\}, f(x, t)$ is a measurable function defined on $\Omega \times \mathbb{R}$ and satisfies:

(a)

$$
|f(x, t)| \leq C_{1}+C_{2}|t|^{\sigma}
$$

for $x \in \Omega$ and $t \in \mathbb{R}$, where $0<\sigma<\frac{m+2}{m-2}$ for $m \geq 3, \sigma>0$ for $m=1,2$ and $C_{1}, C_{2}>0$ are two constants,

(b) $G(t v) \geq 0$ and $G(t v)=o\left(t^{2}\right)$ as $t \rightarrow 0$ and $t \rightarrow \infty$, for every $v \in S_{L^{\perp}}$.

Then, the peak mapping

$$
P(v)=\left\{t v \mid t>0 \text { such that } J(t v)=\max _{s \geq 0} J(s v)\right\}
$$

is well defined on $S_{L^{\perp}}$, i.e., $P(v) \neq \emptyset$ for each $v \in S_{L^{\perp}}$.

Proof. First, similar to Lemma 3.6, assumption (a) guarantees that $J$ (4.1) is locally Lipschitz continuous on $H_{0}^{1}(\Omega)$. Then, denote $I(t)=J(t v)$ for $v \in S_{L^{\perp}}$. Since $G(t v)=o\left(t^{2}\right)$ as $t \rightarrow 0$, we have

$$
I(t)=t^{2}\left(\frac{1}{2} \int_{\Omega}|\nabla v(x)|^{2} d x-\frac{G(t v)}{t^{2}}\right)=t^{2}\left(\frac{1}{2}-\frac{G(t v)}{t^{2}}\right)>0
$$

as $t>0$ is small and since $G(t v) \geq 0$ and $G(t v)=o\left(t^{2}\right)$ as $t \rightarrow \infty$, we have

$$
I(t)=t^{2}\left(\frac{1}{2}-\frac{G(t v)}{t^{2}}\right) \rightarrow-\infty
$$

as $t \rightarrow+\infty$. Thus, by the continuity of $I(t)$ and $I(0)=0$, there is at least one $t_{v}>0$ such that $t_{v}$ is a local maximum point of $I(t)$, i.e.,

$$
P(v)=\left\{t v \mid t>0 \text { such that } J(t v)=\max _{s \geq 0} J(s v)\right\} \neq \emptyset .
$$

Remark 4.2. For our numerical example, by (4.2),

$$
|f(x, t)| \leq C_{1}+C_{2}|t|^{\alpha-1},
$$

where $C_{1}=c \beta b^{r}, C_{2}=c \alpha b^{r}$ and $b$ is a bound of $\Omega$ and by (4.3), $G(u)=$ $\int_{\Omega} F(x, u(x)) d x$ satisfies that $G(t v) \geq 0$ and $G(t v)=o\left(t^{2}\right)$ as $t \rightarrow 0$ and $t \rightarrow \infty$, for every $v \in S_{L^{\perp}}$. 
Lemma 4.3. Assume that $L=\{0\}, J$ (4.1) is locally Lipschitz continuous on $H_{0}^{1}(\Omega)$, the peak mapping $P(v)=\left\{t v \mid t>0\right.$ such that $\left.J(t v)=\max _{s \geq 0} J(s v)\right\}$ is well defined on $S_{L^{\perp}}, F(x, t)$ is regular (cf. [7]) at every $t \in \mathbb{R}$ for $x \in \Omega$ and satisfies that $\frac{\partial F(x, t)}{t}$ is monotone in the sense that for any $\left|t_{2}\right|>\left|t_{1}\right|>0$, any $\delta_{1} F\left(x, t_{1}\right) \in \partial F\left(x, t_{1}\right)$ and $\delta_{2} F\left(x, t_{2}\right) \in \partial F\left(x, t_{2}\right)$,

$$
\frac{\delta_{2} F\left(x, t_{2}\right)}{t_{2}}>\frac{\delta_{2} F\left(x, t_{1}\right)}{t_{1}} \text { for } x \in \Omega \text { and } x \neq x_{1}, \ldots, x_{k},
$$

where $x_{1}, \ldots, x_{k} \in \Omega$ and $\partial F(x, t)$ is generalized gradient of $F(x, \cdot)$ at $t$ for fixed $x \in \Omega$. Then, the peak mapping

$$
P(v)=\left\{t v \mid t>0 \text { such that } J(t v)=\max _{s \geq 0} J(s v)\right\}
$$

is single-valued for every $v \in S_{L^{\perp}}$, i.e., for every $v \in S_{L^{\perp}}, P(v)=\{p(v)\}$, where $p(v)=t_{v} v$ for some $t_{v}>0$.

Proof. Since $F(x, t)$ is regular at every $t \in \mathbb{R}$ for $x \in \Omega$, we have

$$
\partial I(t)=\left\{t-\int_{\Omega} \delta F(x, t v(x)) v(x) d x|\delta F(x, t v(x)) \in \partial F(x, s)|_{s=t v(x)}\right\},
$$

where $I(t)=J(t v)$ for every $v \in S_{L^{\perp}}$. Hence, $0 \in \partial I\left(t_{v}\right)$, where $t_{v} v \in P(v)$, i.e., there is $\left.\delta F\left(x, t_{v} v(x)\right) \in \partial F(x, s)\right|_{s=t_{v} v(x)}$ such that

$$
\int_{\Omega} \frac{\delta F\left(x, t_{v} v(x)\right)}{t_{v} v(x)} v^{2}(x) d x=1 .
$$

By monotonicity of $\frac{\partial F(x, t)}{t}$, if $t_{0}>t_{v}$,

$$
\int_{\Omega} \frac{\delta F\left(x, t_{0} v(x)\right)}{t_{0} v(x)} v^{2}(x) d x>\int_{\Omega} \frac{\delta F\left(x, t_{v} v(x)\right)}{t_{v} v(x)} v^{2}(x) d x=1
$$

and if $0<t_{0}<t_{v}$,

$$
\int_{\Omega} \frac{\delta F\left(x, t_{0} v(x)\right)}{t_{0} v(x)} v^{2}(x) d x<\int_{\Omega} \frac{\delta F\left(x, t_{v} v(x)\right)}{t_{v} v(x)} v^{2}(x) d x=1 .
$$

Thus, such $t_{v}>0$ is unique, i.e, the peak mapping $P$ is single-valued for every $v \in S_{L^{\perp}}$.

Remark 4.4. (a) To our numerical example,

$$
\partial F(x, t)= \begin{cases}\left\{c \alpha|x|^{r}|t|^{\alpha-2} t\right\}, & \text { if }|t|>1 \\ \left\{c \beta|x|^{r}|t|^{\beta-2} t\right\}, & \text { if }|t|<1 \\ {\left[c \beta|x|^{r}, c \alpha|x|^{r}\right],} & \text { if } t=1, \\ {\left[-c \alpha|x|^{r},-c \beta|x|^{r}\right],} & \text { if } t=-1\end{cases}
$$

Hence, for any $\left|t_{2}\right|>\left|t_{1}\right|$, any $\delta_{1} F\left(x, t_{1}\right) \in \partial F\left(x, t_{1}\right)$ and $\delta_{2} F\left(x, t_{2}\right) \in \partial F\left(x, t_{2}\right)$,

$$
\frac{\delta_{2} F\left(x, t_{2}\right)}{t_{2}}>\frac{\delta_{2} F\left(x, t_{1}\right)}{t_{1}} \text { for } x \in \Omega \text { and } x \neq 0 .
$$

(b) Lemma 4.1 and Lemma 4.3 guarantee that

$$
\inf _{v \in S_{L^{\perp}}} J(p(v)) \geq 0,
$$


where $p$ is the peak selection of $J$ (4.1) w.r.t. $L=\{0\}$ with $p(v)=t v, t>0$ for $v \in S_{L^{\perp}}$, under assumptions in Lemma 4.1 and Lemma 4.3. Indeed, if the peak mapping of $J$ (4.1) w.r.t. a finite dimensional space $L \subset H_{0}^{1}(\Omega)$,

$$
P(v)=\left\{t v+w \mid t>0 \text { and } w \in L \text { such that } J(t v+w)=\max _{s \geq 0, u \in L} J(s v+u)\right\},
$$

is well defined, then,

$$
\inf _{v \in S_{L \perp}} J(p(v)) \geq 0,
$$

where $p$ is a peak selection of $J$ (4.1) w.r.t. $L$ such that $p(v) \in P(v)$ for every $v \in S_{L^{\perp}}$, under assumptions in Lemma 4.1 and Lemma 4.3 .

Lemma 4.5. Assume that $L=\{0\}, J$ (4.1) is locally Lipschitz continuous on $H_{0}^{1}(\Omega)$, the peak mapping $P(v)=\left\{t v \mid t>0\right.$ such that $\left.J(t v)=\max _{s \geq 0} J(s v)\right\}$ is well defined on $S_{L^{\perp}}, P\left(v_{0}\right)=\left\{u_{0}\right\}$ where $v_{0} \in S_{L^{\perp}}, t=0$ is not a maximum point of $J\left(t v_{0}\right)$ on $\{t \in \mathbb{R} \mid t \geq 0\}, F(x, t)=F_{1}(x) F_{2}(t), 0 \leq F_{1}(x) \leq C$ for every $x \in \Omega$ where $C>0$ is a constant, $F_{2}(t)$ is regular at every $t \in \mathbb{R}$ and satisfies that, for every $\delta F_{2}(t) \in \partial F_{2}(t)$,

$$
\delta F_{2}(t) t \geq a_{1}|t|^{\mu}+a_{2},
$$

where $\mu>2, \mu<\frac{2 m}{m-2}$ for $m \geq 3$ and $a_{1}>0, a_{2}$ are two constants. Then, any peak selection $p(v)$ is continuous at $v_{0}$, where $p(v) \in P(v)$ for $v \in S_{L^{\perp}}$ and $p(v)$ is a global maximum point of $J$ (4.1) on $\{t v \mid t \geq 0\}$ for $v \in S_{L^{\perp}}$ around $v_{0}$.

Proof. Since $F_{2}(t)$ is regular at every $t \in \mathbb{R}$, we have

$$
\partial I(t)=\left\{t-\int_{\Omega} F_{1}(x) \delta F_{2}(t v(x)) v(x) d x\left|\delta F_{2}(t v(x)) \in \partial F_{2}(s)\right|_{s=t v(x)}\right\},
$$

where $I(t)=J(t v)$ for $v \in S_{L^{\perp}}$. Suppose that $v_{n} \rightarrow v_{0}$. Denote that $p\left(v_{n}\right)=t_{n} v_{n}$, $t_{n}>0$ and $p\left(v_{0}\right)=t_{0} v_{0}, t_{0}>0$. Then, $0 \in \partial I_{n}\left(t_{n}\right)$, where $I_{n}(t)=J\left(t v_{n}\right)$. By (4.6), there is $\left.\delta F_{2}\left(t_{n} v_{n}(x)\right) \in \partial F_{2}(s)\right|_{s=t_{n} v_{n}(x)}$ such that

$$
t_{n}-\int_{\Omega} F_{1}(x) \delta F_{2}\left(t_{n} v_{n}(x)\right) v_{n}(x) d x=0 .
$$

Thus,

$$
1=\frac{1}{t_{n}^{2}} \int_{\Omega} F_{1}(x) \delta F_{2}\left(t_{n} v_{n}(x)\right) t_{n} v_{n}(x) d x \geq \frac{1}{t_{n}^{2}} \int_{\Omega} F_{1}(x)\left(a_{1}\left|t_{n} v_{n}(x)\right|^{\mu}+a_{2}\right) d x,
$$

i.e.,

$$
1 \geq a_{1}\left\|p\left(v_{n}\right)\right\|^{\mu-2} \int_{\Omega} F_{1}(x)\left|v_{n}(x)\right|^{\mu} d x-\left\|p\left(v_{n}\right)\right\|^{-2} C|\Omega| a_{2},
$$

where $|\Omega|$ is the measure of $\Omega$. Suppose that $\left\{t_{n}\right\}$ is unbounded. Then, there is $\left\{t_{n_{k}}\right\}$ such that $t_{n_{k}} \rightarrow+\infty$, i.e., $\left\|p\left(v_{n_{k}}\right)\right\| \rightarrow+\infty$. Since $v_{n} \rightarrow v_{0}$, this contradicts (4.7). Hence, $\left\{t_{n}\right\}$ is bounded. Without loss of generality, we can assume that $t_{n} \rightarrow \bar{t}$. Then, since $p\left(v_{n}\right)$ is a global maximum point of $J$ (4.1) on $\left\{t v_{n} \mid t \geq 0\right\}$ for large $n, \bar{t} v_{0}$ is a maximum point of $J$ (4.1) on $\left\{t v_{0} \mid t \geq 0\right\}$. On the other hand, Since $P\left(v_{0}\right)=\left\{u_{0}\right\}$ and $t=0$ is not a maximum point of $J\left(t v_{0}\right)$ on $\{t \in \mathbb{R} \mid t \geq 0\}$, $u_{0}=t_{0} v_{0}=\bar{t} v_{0}$. Hence, $\bar{t}=t_{0}$, i.e., $p\left(v_{n}\right) \rightarrow p\left(v_{0}\right)$, i.e., $p(v)$ is continuous at $v_{0}$. 
Remark 4.6. (a) For our numerical example, $F_{1}(x)=|x|^{r}, r>0$,

$$
F_{2}(t)=\left\{\begin{array}{l}
|t|^{\alpha}, \text { if }|t|>1, \\
|t|^{\beta}, \text { if }|t| \leq 1
\end{array}\right.
$$

and

$$
\partial F_{2}(t)= \begin{cases}\left\{c \alpha|t|^{\alpha-2} t\right\}, & \text { if }|t|>1, \\ \left\{c \beta|t|^{\beta-2} t\right\}, & \text { if }|t|<1, \\ {[c \beta, c \alpha],} & \text { if } t=1, \\ {[-c \alpha,-c \beta],} & \text { if } t=-1 .\end{cases}
$$

Thus, for every $\delta F_{2}(t) \in \partial F_{2}(t)$,

$$
\delta F_{2}(t) t \geq a_{1}|t|^{\beta}+a_{2},
$$

where $a_{1}=c \beta$ and $a_{2}=0$.

(b) For our numerical example, Lemma 4.1 Lemma 4.3 and Lemma 4.5 guarantee that the peak selection $p(v)=t v$, where $t>0$ and $J(t v)=\max _{s \geq 0} J(s v)$, is continuous on $S_{L^{\perp}}$, where $L=\{0\}$.

Lemma 4.5 can be extended to the following theorem and the theorem can be verified in the same way as Theorem 4.1 in [10. On the other hand, this theorem extends Theorem 4.1 in [10].

Theorem 4.7. Assume that $L \subset H_{0}^{1}(\Omega)$ is a finite dimensional space, $J$ (4.1) is locally Lipschitz continuous on $H_{0}^{1}(\Omega)$, the peak mapping of $J$ (4.1) w.r.t. L,

$$
P(v)=\left\{t v+w \mid t>0 \text { and } w \in L \text { such that } J(t v+w)=\max _{s \geq 0, u \in L} J(s v+u)\right\},
$$

is well defined and $P\left(v_{0}\right)=\left\{u_{0}\right\}$ where $v_{0} \in S_{L^{\perp}},(0, w)$ is not a maximum point of $J\left(t v_{0}+u\right)$ on $\{t \in \mathbb{R} \mid t \geq 0\} \times L$ for any $w \in L, F(x, t)=F_{1}(x) F_{2}(t), 0 \leq F_{1}(x) \leq C$ for $x \in \Omega$ where $C>0$ is a constant, $F_{2}(t)$ is regular at every $t \in \mathbb{R}$ and satisfies that, for every $\delta F_{2}(t) \in \partial F_{2}(t)$,

$$
\delta F_{2}(t) t \geq a_{1}|t|^{\mu}+a_{2}, \forall x \in \Omega,
$$

where $\mu>2, \mu<\frac{2 m}{m-2}$ for $m \geq 3$ and $a_{1}>0, a_{2}$ are two constants. Then, any peak selection $p(v)$ is continuous at $v_{0}$, where $p(v) \in P(v)$ for $v \in S_{L^{\perp}}$ and $p(v)$ is a global maximum point of $J$ (4.1) on $\{t v+w \mid t \geq 0, w \in L\}$ for $v \in S_{L^{\perp}}$ around $v_{0}$.

Lemma 4.8. Assume that $L=\{0\}, J$ (4.1) is locally Lipschitz continuous on $H_{0}^{1}(\Omega)$, the peak mapping $P(v)=\left\{t v \mid t>0\right.$ such that $\left.J(t v)=\max _{s \geq 0} J(s v)\right\}$ is well defined on $S_{L^{\perp}}, F(x, t)$ is regular at every $t \in \mathbb{R}$ for $x \in \Omega$ and satisfies that, for every $\delta F(x, t) \in \partial F(x, t)$ where $\partial F(x, t)$ is generalized gradient of $F(x, \cdot)$ at $t$ for fixed $x \in \Omega$,

$$
\delta F(x, t) t \leq a t^{2}+\sum_{i=1}^{k} b_{i}|t|^{\rho_{i}}, \forall x \in \Omega,
$$

where $0 \leq a<\lambda_{1}, \lambda_{1}$ is the first eigenvalue of $\Delta$ on $\Omega, \rho_{1}, \ldots, \rho_{k}>2, \rho_{1}, \ldots, \rho_{k}<$ $\frac{2 m}{m-2}$ for $m \geq 3$ and $\rho_{1}, \ldots, \rho_{k}, b_{1}>0, \ldots, b_{k}>0$ are constants. Then, there is $\alpha>0$ such that $d(p(v), L) \geq \alpha$, where $p(v)$ is a peak selection such that $p(v) \in P(v)$ for $v \in S_{L^{\perp}}$. 
Proof. Since $F(x, t)$ is regular at every $t \in \mathbb{R}$ for $x \in \Omega$, we have

$$
\partial I(t)=\left\{t-\int_{\Omega} \delta F(x, t v(x)) v(x) d x|\delta F(x, t v(x)) \in \partial F(x, s)|_{s=t v(x)}\right\},
$$

where $I(t)=J(t v)$ for $v \in S_{L^{\perp}}$. On the other hand, according to (4.8), we have

$$
\begin{aligned}
& \int_{\Omega} \delta F(x, u(x)) u(x) d x \leq \int_{\Omega}\left(a(u(x))^{2}+\sum_{i=1}^{k} b_{i}|u(x)|^{\rho_{i}}\right) d x \\
\leq & a \lambda_{1}^{-1} \int_{\Omega}|\nabla u(x)|^{2} d x+\sum_{i=1}^{k} b_{i} c_{i}\left(\int_{\Omega}|\nabla u(x)|^{2} d x\right)^{\frac{\rho_{i}}{2}} \\
= & \left(a \lambda_{1}^{-1}+\sum_{i=1}^{k} b_{i} c_{i}\|u\|^{\rho_{i}-2}\right)\|u\|^{2},
\end{aligned}
$$

where $u=t v,\left.\delta F(x, u(x)) \in \partial F(x, s)\right|_{s=u(x)}$ and $c_{1}, \ldots, c_{k}$ are constants. Thus, as $t>0$ is small,

$$
\delta I(t) \geq\left(1-a \lambda_{1}+\sum_{i=1}^{k} b_{i} c_{i} t^{\rho_{i}-2}\right) t>0
$$

for any $\delta I(t) \in \partial I(t)$. Hence, there is $\alpha>0$ such that $d(p(v), L)=\|p(v)\| \geq \alpha$ for any peak selection $p(v)$ such that $p(v) \in P(v)$ for $v \in S_{L^{\perp}}$.

Remark 4.9. To our numerical example, $\partial F(x, t)$ is given by (4.5). Hence, for every $\delta F(x, t) \in \partial F(x, t)$,

$$
\delta F(x, t) t \leq a t^{2}+b_{1}|t|^{\beta}+b_{2}|t|^{\alpha}, \forall x \in \Omega,
$$

where $a=0, b_{1}=c \beta b^{r}, b_{2}=c \alpha b^{r}$ and $b$ is a bound of $\Omega$. Hence, to our numerical example, there is $\alpha>0$ such that

$$
d(p(v), L) \geq \alpha
$$

where $p$ is the peak selection of $J$ (4.1) w.r.t. $L=\{0\}$ with $p(v)=t v, t>0$ for $v \in S_{L^{\perp}}$.

To super-linear property of peak selection to $J$ (4.1) with $f(x, t)$ (4.2), we have Theorem 4.18. For verifying this theorem, we need Lemma 4.10-Lemma 4.16 to get Theorem 4.17. Then, Theorem 4.18 can be obtained by Theorem 4.17 .

Lemma 4.10. Assume that $L=\{0\}, f(x, t)$ is in (4.2), $p$ is a peak selection of $J$ (4.1) defined on $S_{L^{\perp}}, p(w)=t_{w} w, t_{w}>0$ for $w \in S_{L^{\perp}}, p$ is continuous at $v \in S_{L^{\perp}}$ and $\left\{v_{n}\right\} \subset S_{L^{\perp}}$ satisfies $\left\|v_{n}-v\right\| \leq 1, v_{n} \rightarrow v$ and

$$
\frac{\left|t_{n}-t\right|}{\left\|v_{n}-v\right\|^{\beta_{0}}} \rightarrow+\infty
$$


where $p\left(v_{n}\right)=t_{n} v_{n}, t_{n}>0, p(v)=t v, t>0$ and $\beta_{0}=\frac{1}{2}+\alpha_{0}, \alpha_{0} \in\left(0, \frac{1}{6}\right]$. Denote

$$
\begin{gathered}
A_{1}^{n}=\left\{x \in \Omega|| t v(x)|>1,| t_{n} v_{n}(x) \mid>1\right\}, \\
A_{2}^{n}=\left\{x \in \Omega|| t v(x)|>1,| t_{n} v_{n}(x) \mid=1\right\}, \quad A_{3}^{n}=\left\{x \in \Omega|| t v(x)|>1,| t_{n} v_{n}(x) \mid<1\right\}, \\
B_{1}^{n}=\left\{x \in \Omega|| t v(x)|=1,| t_{n} v_{n}(x) \mid>1\right\}, \\
B_{2}^{n}=\left\{x \in \Omega|| t v(x)|=1,| t_{n} v_{n}(x) \mid=1\right\}, \quad B_{3}^{n}=\left\{x \in \Omega|| t v(x)|=1,| t_{n} v_{n}(x) \mid<1\right\}, \\
C_{1}^{n}=\left\{x \in \Omega|| t v(x)|<1,| t_{n} v_{n}(x) \mid>1\right\}, \\
C_{2}^{n}=\left\{x \in \Omega|| t v(x)|<1,| t_{n} v_{n}(x) \mid=1\right\}, \quad C_{3}^{n}=\left\{x \in \Omega|| t v(x)|<1,| t_{n} v_{n}(x) \mid<1\right\} .
\end{gathered}
$$

Thus,

(a) if $A_{2}^{n} \cup A_{3}^{n} \cup B_{3}^{n} \neq \emptyset$ for $n=1,2, \ldots$, then, either there is $\left\{t_{n_{k}}\right\}$ such that $t>t_{n_{k}}$ or there is $\left\{t_{n_{k}}\right\}$ and a constant $C>0$ such that $t<t_{n_{k}}$ and

$$
\int_{\Omega_{n_{k}}}\left|t_{n_{k}} v_{n_{k}}(x)\right| d x \leq C s_{n_{k}}^{\beta_{0}}
$$

where $\Omega_{n_{k}}=A_{2}^{n_{k}} \cup A_{3}^{n_{k}} \cup B_{3}^{n_{k}}$,

(b) if $B_{2}^{n} \neq \emptyset$ for $n=1,2, \ldots$, then, either there is $\left\{t_{n_{k}}\right\}$ such that $t>t_{n_{k}}$ or there is $\left\{t_{n_{k}}\right\}$ and a constant $C>0$ such that $t<t_{n_{k}}$ and

$$
\int_{B_{2}^{n_{k}}}\left|t_{n_{k}} v_{n_{k}}(x)\right| d x \leq C s_{n_{k}}^{\beta_{0}}
$$

where $v_{n_{k}}=\frac{1}{\sqrt{1+s_{n_{k}}^{2}}}\left(v-s_{n_{k}} d_{n_{k}}\right),\left\|d_{n_{k}}\right\|=1,\left\langle d_{n_{k}}, v\right\rangle=0$ and $s_{n_{k}} \geq 0$.

Proof. (a) Since $\left\|v_{n}-v\right\| \leq 1$ and

$$
\left\|v_{n}-v\right\|^{2}=\left\langle v_{n}-v, v_{n}-v\right\rangle=\left\|v_{n}\right\|^{2}+\|v\|^{2}-2\left\langle v_{n}, v\right\rangle=2-2\left\langle v_{n}, v\right\rangle,
$$

we have $\left\langle v_{n}, v\right\rangle \geq \frac{1}{2}$. By Lemma 2.12, there is unique $d_{n}$ with $\left\|d_{n}\right\|=1,\left\langle d_{n}, v\right\rangle=0$ and $s_{n} \geq 0$ such that

$$
v_{n}=\frac{1}{\sqrt{1+s_{n}^{2}}}\left(v-s_{n} d_{n}\right)
$$

Then,

$$
v_{n}-v=-\frac{s_{n}^{2}}{\left(\sqrt{1+s_{n}^{2}}\right)\left(1+\sqrt{1+s_{n}^{2}}\right)} v-\frac{s_{n}}{\sqrt{1+s_{n}^{2}}} d_{n}
$$

i.e.,

$$
\left\|v_{n}-v\right\|=\frac{s_{n}}{\sqrt{1+s_{n}^{2}}} \sqrt{\frac{s_{n}^{2}}{\left(1+\sqrt{1+s_{n}^{2}}\right)^{2}}+1}
$$

Thus,

$$
\lim _{n \rightarrow \infty} \frac{\left\|v_{n}-v\right\|}{s_{n}}=1 .
$$

Hence, by (4.9),

$$
D_{n}=\frac{\left|t_{n}-t\right|}{s_{n}^{\beta_{0}}} \rightarrow+\infty
$$


By (4.10), we have

$$
\begin{aligned}
& t_{n} v_{n} \\
= & \frac{1}{\sqrt{1+s_{n}^{2}}}\left(t_{n} v-t_{n} d_{n} s_{n}\right)=\left(t_{n} v-t_{n} d_{n} s_{n}\right)+\left(\frac{1}{\sqrt{1+s_{n}^{2}}}-1\right)\left(t_{n} v-t_{n} d_{n} s_{n}\right) \\
= & t v-\left(t-t_{n}\right) v-t_{n} d_{n} s_{n}-\frac{\left(t_{n} v-t_{n} d_{n} s_{n}\right) s_{n}^{2}}{f\left(s_{n}\right)}
\end{aligned}
$$

i.e.,

$$
t_{n} v_{n}=t v-\left(t-t_{n}\right) v-t_{n} d_{n} s_{n}-\frac{t_{n} v s_{n}^{2}}{f\left(s_{n}\right)}+\frac{t_{n} d_{n} s_{n}^{3}}{f\left(s_{n}\right)},
$$

where $f\left(s_{n}\right)=\sqrt{1+s_{n}^{2}}\left(1+\sqrt{1+s_{n}^{2}}\right)$.

For every $x \in A_{2}^{n} \cup A_{3}^{n}$, we have either $t v(x)>1,\left|t_{n} v_{n}(x)\right| \leq 1$ or $t v(x)<-1$, $\left|t_{n} v_{n}(x)\right| \leq 1$. If $t v(x)>1,\left|t_{n} v_{n}(x)\right| \leq 1$ and $t<t_{n}$, then, by (4.11) and (4.12), there is $n_{1}>0$ such that $n_{1}$ is independent of $x$ and as $n>n_{1}$,

$$
\begin{aligned}
& -\frac{1}{2}\left(t-t_{n}\right) v(x)-2 t_{n}\left|d_{n}(x)\right| s_{n} \\
\leq & -\left(t-t_{n}\right) v(x)-t_{n} d_{n}(x) s_{n}-\frac{t_{n} v(x) s_{n}^{2}}{f\left(s_{n}\right)}-t_{n}\left|d_{n}(x)\right| s_{n} \\
\leq & -\left(t-t_{n}\right) v(x)-t_{n} d_{n}(x) s_{n}-\frac{t_{n} v(x) s_{n}^{2}}{f\left(s_{n}\right)}+\frac{t_{n} d_{n}(x) s_{n}^{3}}{f\left(s_{n}\right)} \\
= & t_{n} v_{n}(x)-t v(x)<0,
\end{aligned}
$$

i.e.,

$$
\left|t_{n}-t\right||v(x)|<4 t_{n}\left|d_{n}(x)\right| s_{n} .
$$

If $t v(x)<-1,\left|t_{n} v_{n}(x)\right| \leq 1$ and $t<t_{n}$, then, by (4.11) and (4.12), there is $n_{2}>0$ such that $n_{2}$ is independent of $x$ and as $n>n_{2}$,

$$
\begin{aligned}
& -\frac{1}{2}\left(t-t_{n}\right) v(x)+2 t_{n}\left|d_{n}(x)\right| s_{n} \\
> & -\frac{1}{2}\left(t-t_{n}\right) v(x)-t_{n} d_{n}(x) s_{n}+\frac{t_{n} d_{n}(x) s_{n}^{3}}{f\left(s_{n}\right)} \\
> & -\left(t-t_{n}\right) v(x)-t_{n} d_{n}(x) s_{n}-\frac{t_{n} v(x) s_{n}^{2}}{f\left(s_{n}\right)}+\frac{t_{n} d_{n}(x) s_{n}^{3}}{f\left(s_{n}\right)} \\
= & t_{n} v_{n}(x)-t v(x)>0,
\end{aligned}
$$

i.e.,

$$
\left|t_{n}-t\right||v(x)|<4 t_{n}\left|d_{n}(x)\right| s_{n} .
$$

Thus, $\forall x \in A_{2}^{n} \cup A_{3}^{n}$, if $t<t_{n}$ and $n>\max \left(n_{1}, n_{2}\right)$, we have

$$
\left|t_{n}-t\right||v(x)|<4 t_{n}\left|d_{n}(x)\right| s_{n} .
$$

Similarly, $\forall x \in B_{3}^{n}$, if $t<t_{n}$, there is $n_{3}>0$ such that $n_{3}$ is independent of $x$ and as $n>n_{3}$, we have

$$
\left|t_{n}-t\right||v(x)|<4 t_{n}\left|d_{n}(x)\right| s_{n} .
$$

Hence, $\forall x \in A_{2}^{n} \cup A_{3}^{n} \cup B_{3}^{n}$, if $t<t_{n}$ and $n>\max \left(n_{1}, n_{2}, n_{3}\right)$, we have

$$
\left|t_{n}-t\right||v(x)|<4 t_{n}\left|d_{n}(x)\right| s_{n} .
$$


The inequality (4.13) means, if $t<t_{n}$ and $n$ is large,

$$
\left|t_{n}-t\right| \int_{\Omega_{n}}|v(x)| d x<4 t_{n} s_{n} \int_{\Omega_{n}}\left|d_{n}(x)\right| d x
$$

where $\Omega_{n}=A_{2}^{n} \cup A_{3}^{n} \cup B_{3}^{n}$. By (4.14), if $t<t_{n}$ and $n$ is large,

$$
\begin{aligned}
& \frac{\left|t_{n}-t\right|}{s_{n}^{\beta_{0}}}<4 s_{n}^{-2 \alpha_{0}} t_{n} \int_{\Omega_{n}}\left|d_{n}(x)\right| d x\left(\frac{\int_{\Omega_{n}}|v(x)| d x}{s_{n}^{\beta_{0}}}\right)^{-1} \\
\leq & 4 s_{n}^{-2 \alpha_{0}} t_{n}\left(\int_{\Omega_{n}}\left|d_{n}(x)\right|^{2} d x\right)^{\frac{1}{2}}\left(\int_{\Omega_{n}} 1^{2} d x\right)^{\frac{1}{2}}\left(\frac{\int_{\Omega_{n}}|v(x)| d x}{s_{n}^{\beta_{0}}}\right)^{-1} \\
\leq & 4 s_{n}^{-2 \alpha_{0}} t_{n} t\left(\int_{\Omega_{n}}\left|d_{n}(x)\right|^{2} d x\right)^{\frac{1}{2}}\left|\Omega_{n}\right|^{\frac{1}{2}}\left(\frac{\int_{\Omega_{n}}|t v(x)| d x}{s_{n}^{\beta_{0}}}\right)^{-1} \\
\leq & 4 s_{n}^{-2 \alpha_{0}} t_{n} t \lambda_{1}^{-1}\left|\Omega_{n}\right|^{\frac{1}{2}}\left(\frac{\int_{\Omega_{n}}|t v(x)| d x}{s_{n}^{\beta_{0}}}\right)^{-1},
\end{aligned}
$$

where $\lambda_{1}$ is the first eigenvalue of $\Delta$ on $\Omega$ and $\left|\Omega_{n}\right|$ is the measure of $\Omega_{n}$, and by (4.11) and (4.14), if $t<t_{n}$ and $n$ is large,

$$
\begin{aligned}
\left|\Omega_{n}\right| & =\int_{\Omega_{n}} d x \leq \int_{\Omega_{n}}|t v(x)| d x<4 t_{n} t \frac{s_{n}^{\beta_{0}}}{\left|t_{n}-t\right|} s_{n}^{1-\beta_{0}} \int_{\Omega_{n}}\left|d_{n}(x)\right| d x \\
& =4 s_{n}^{\frac{1}{2}-\alpha_{0}} t_{n} t D_{n}^{-1} \int_{\Omega_{n}}\left|d_{n}(x)\right| d x \leq 4 s_{n}^{\frac{1}{2}-\alpha_{0}} t_{n} t D_{n}^{-1} \lambda_{1}^{-1}\left|\Omega_{n}\right|^{\frac{1}{2}},
\end{aligned}
$$

i.e.,

$$
\left|\Omega_{n}\right| \leq s_{n}^{1-2 \alpha_{0}}
$$

Then, if $t<t_{n}$ and $n$ is large,

$$
\frac{\left|t_{n}-t\right|}{s_{n}^{\beta_{0}}} \leq 4 s_{n}^{\frac{1}{2}-3 \alpha_{0}} t_{n} t \lambda_{1}^{-1}\left(\frac{\int_{\Omega_{n}}|t v(x)| d x}{s_{n}^{\beta_{0}}}\right)^{-1},
$$

i.e.,

$$
\frac{\int_{\Omega_{n}}|t v(x)| d x}{s_{n}^{\beta_{0}}} \leq 4 s_{n}^{\frac{1}{2}-3 \alpha_{0}} t_{n} t \lambda_{1}^{-1}\left(\frac{\left|t_{n}-t\right|}{s_{n}^{\beta_{0}}}\right)^{-1}=4 s_{n}^{\frac{1}{2}-3 \alpha_{0}} t_{n} t \lambda_{1}^{-1} D_{n}^{-1} .
$$

Thus, since $\alpha_{0} \in\left(0, \frac{1}{6}\right]$, by (4.11), if $t<t_{n}$ and $n$ is large,

$$
\int_{\Omega_{n}}|t v(x)| d x \leq D s_{n}^{\beta_{0}}
$$

where $D$ is a constant. By (4.10) and (4.15), if $t<t_{n}$ and $n$ is large, then

$$
\begin{aligned}
& \int_{\Omega_{n}}\left|t_{n} v_{n}(x)\right| d x=\frac{t_{n}}{\sqrt{1+s_{n}^{2}}} \int_{\Omega_{n}}\left|\sqrt{1+s_{n}^{2}} v_{n}(x)\right| d x \\
\leq & \frac{t_{n}}{\sqrt{1+s_{n}^{2}}}\left(\int_{\Omega_{n}}\left|\sqrt{1+s_{n}^{2}} v_{n}(x)+s_{n} d_{n}(x)\right| d x+\int_{\Omega_{n}}\left|s_{n} d_{n}(x)\right| d x\right) \\
\leq & \frac{t_{n}}{\sqrt{1+s_{n}^{2}}}\left(t^{-1} \int_{\Omega_{n}}|t v(x)| d x+s_{n}\left|\Omega_{n}\right|^{\frac{1}{2}}\left(\int_{\Omega_{n}}\left|d_{n}(x)\right|^{2} d x\right)^{\frac{1}{2}}\right) \\
\leq & \frac{t_{n}}{\sqrt{1+s_{n}^{2}}}\left(t^{-1} D s_{n}^{\beta_{0}}+s_{n}\left|\Omega_{n}\right|^{\frac{1}{2}} \lambda_{1}^{-1}\right) \leq C s_{n}^{\beta_{0}},
\end{aligned}
$$


where $C>0$ is a constant. Hence, either there is $\left\{t_{n_{k}}\right\}$ such that $t>t_{n_{k}}$ or there is $\left\{t_{n_{k}}\right\}$ and a constant $C>0$ such that $t<t_{n_{k}}$ and

$$
\int_{\Omega_{n_{k}}}\left|t_{n_{k}} v_{n_{k}}(x)\right| d x \leq C s_{n_{k}}^{\beta_{0}},
$$

where $\Omega_{n_{k}}=A_{2}^{n_{k}} \cup A_{3}^{n_{k}} \cup B_{3}^{n_{k}}$.

(b) The conclusion can be verified in the same way as (a).

Lemma 4.11. Assume that $L=\{0\}, f(x, t)$ is in (4.2), $p$ is a peak selection of $J$ (4.1) defined on $S_{L^{\perp}}, p(w)=t_{w} w, t_{w}>0$ for $w \in S_{L^{\perp}}, p$ is continuous at $v \in S_{L^{\perp}}$ and $\left\{v_{n}\right\} \subset S_{L^{\perp}}$ satisfies $\left\|v_{n}-v\right\| \leq 1, v_{n} \rightarrow v$ and

$$
\frac{\left|t_{n}-t\right|}{\left\|v_{n}-v\right\|^{\beta_{0}}} \rightarrow+\infty
$$

where $p\left(v_{n}\right)=t_{n} v_{n}, t_{n}>0, p(v)=t v, t>0$ and $\beta_{0}=\frac{1}{2}+\alpha_{0}, \alpha_{0} \in\left(0, \frac{1}{6}\right]$. $A_{i}^{n}, B_{i}^{n}, C_{i}^{n}, i=1,2,3$, are defined in Lemma 4.10. Thus,

(a) if $C_{1}^{n} \cup C_{2}^{n} \cup B_{1}^{n} \neq \emptyset$ for $n=1,2, \ldots$, then, either there is $\left\{t_{n_{k}}\right\}$ such that $t<t_{n_{k}}$ or there is $\left\{t_{n_{k}}\right\}$ and a constant $C>0$ such that $t>t_{n_{k}}$ and

$$
\int_{\Omega_{n_{k}}}|t v(x)| d x \leq C s_{n_{k}}^{\beta_{0}},
$$

where $\Omega_{n_{k}}=C_{1}^{n_{k}} \cup C_{2}^{n_{k}} \cup B_{1}^{n_{k}}$,

(b) if $B_{2}^{n} \neq \emptyset$ for $n=1,2, \ldots$, then, either there is $\left\{t_{n_{k}}\right\}$ such that $t<t_{n_{k}}$ or there is $\left\{t_{n_{k}}\right\}$ and a constant $C>0$ such that $t>t_{n_{k}}$ and

$$
\int_{B_{2}^{n_{k}}}|t v(x)| d x \leq C s_{n_{k}}^{\beta_{0}},
$$

where $v_{n_{k}}=\frac{1}{\sqrt{1+s_{n_{k}}^{2}}}\left(v-s_{n_{k}} d_{n_{k}}\right),\left\|d_{n_{k}}\right\|=1,\left\langle d_{n_{k}}, v\right\rangle=0$ and $s_{n_{k}} \geq 0$.

Proof. (a) Similar to Lemma 4.10, there is unique $d_{n}$ with $\left\|d_{n}\right\|=1,\left\langle d_{n}, v\right\rangle=0$ and $s_{n} \geq 0$ such that

$$
v_{n}=\frac{1}{\sqrt{1+s_{n}^{2}}}\left(v-s_{n} d_{n}\right)
$$

and

$$
D_{n}=\frac{\left|t_{n}-t\right|}{s_{n}^{\beta_{0}}} \rightarrow+\infty .
$$

By (4.16),

$$
v=\sqrt{1+s_{n}^{2}} v_{n}+s_{n} d_{n}
$$

Then,

$$
t v=\sqrt{1+s_{n}^{2}} t v_{n}+t d_{n} s_{n}=\left(t v_{n}+t d_{n} s_{n}\right)+\left(\sqrt{1+s_{n}^{2}}-1\right) t v_{n}
$$

i.e.,

$$
t v=t_{n} v_{n}+\left(t-t_{n}\right) v_{n}+t d_{n} s_{n}+\frac{t v_{n} s_{n}^{2}}{1+\sqrt{1+s_{n}^{2}}}
$$


For every $x \in C_{1}^{n} \cup C_{2}^{n}$, we have either $|t v(x)|<1, t_{n} v_{n}(x) \geq 1$ or $|t v(x)|<1$, $t_{n} v_{n}(x) \leq-1$. If $|t v(x)|<1, t_{n} v_{n}(x) \geq 1$ and $t>t_{n}$, then, by (4.17) and (4.18), there is $n_{1}>0$ such that $n_{1}$ is independent of $x$ and as $n>n_{1}$,

$$
\begin{aligned}
\left(t-t_{n}\right) v_{n}(x)+t d_{n}(x) s_{n} & \leq\left(t-t_{n}\right) v_{n}(x)+t d_{n}(x) s_{n}+\frac{t v_{n}(x) s_{n}^{2}}{1+\sqrt{1+s_{n}^{2}}} \\
& =t v(x)-t_{n} v_{n}(x)<0
\end{aligned}
$$

i.e.,

$$
\left|t_{n}-t\right|\left|v_{n}(x)\right|<t\left|d_{n}(x)\right| s_{n} .
$$

If $|t v(x)|<1, t_{n} v_{n}(x)<-1$ and $t>t_{n}$, then, (4.17) and (4.18), there is $n_{2}>0$ such that $n_{2}$ is independent of $x$ and as $n>n_{2}$,

$$
\begin{aligned}
\frac{1}{2}\left(t-t_{n}\right) v_{n}(x)+t d_{n}(x) s_{n} & \geq\left(t-t_{n}\right) v_{n}(x)+t d_{n}(x) s_{n}+\frac{t v_{n}(x) s_{n}^{2}}{1+\sqrt{1+s_{n}^{2}}} \\
& =t v(x)-t_{n} v_{n}(x)>0
\end{aligned}
$$

i.e.,

$$
\left|t_{n}-t\right|\left|v_{n}(x)\right|<2 t\left|d_{n}(x)\right| s_{n} .
$$

Thus, $\forall x \in C_{1}^{n} \cup C_{2}^{n}$, if $t>t_{n}$ and $n>\max \left(n_{1}, n_{2}\right)$, we have

$$
\left|t_{n}-t\right|\left|v_{n}(x)\right|<2 t\left|d_{n}(x)\right| s_{n} .
$$

Similarly, $\forall x \in B_{1}^{n}$, if $t>t_{n}$, there is $n_{3}>0$ such that $n_{3}$ is independent of $x$ and as $n>n_{3}$, we have

$$
\left|t_{n}-t\right|\left|v_{n}(x)\right|<2 t\left|d_{n}(x)\right| s_{n} .
$$

Hence, $\forall x \in C_{1}^{n} \cup C_{2}^{n} \cup B_{1}^{n}$, if $t>t_{n}$ and $n>\max \left(n_{1}, n_{2}, n_{3}\right)$, we have

$$
\left|t_{n}-t\right|\left|v_{n}(x)\right|<2 t\left|d_{n}(x)\right| s_{n} .
$$

The inequality means, if $t>t_{n}$ and $n$ is large, then

$$
\left|t_{n}-t\right| \int_{\Omega_{n}}\left|v_{n}(x)\right| d x<2 t s_{n} \int_{\Omega_{n}}\left|d_{n}(x)\right| d x,
$$

where $\Omega_{n}=C_{1}^{n} \cup C_{2}^{n} \cup B_{1}^{n}$. By (4.20), if $t>t_{n}$ and $n$ is large,

$$
\begin{aligned}
& \frac{\left|t_{n}-t\right|}{s_{n}^{\beta_{0}}}<2 s_{n}^{-2 \alpha_{0}} t \int_{\Omega_{n}}\left|d_{n}(x)\right| d x\left(\frac{\int_{\Omega_{n}}\left|v_{n}(x)\right| d x}{s_{n}^{\beta_{0}}}\right)^{-1} \\
\leq & 2 s_{n}^{-2 \alpha_{0}} t\left(\int_{\Omega_{n}}\left|d_{n}(x)\right|^{2} d x\right)^{\frac{1}{2}}\left(\int_{\Omega_{n}} 1^{2} d x\right)^{\frac{1}{2}}\left(\frac{\int_{\Omega_{n}}\left|v_{n}(x)\right| d x}{s_{n}^{\beta_{0}}}\right)^{-1} \\
\leq & 2 s_{n}^{-2 \alpha_{0}} t_{n} t\left(\int_{\Omega_{n}}\left|d_{n}(x)\right|^{2} d x\right)^{\frac{1}{2}}\left|\Omega_{n}\right|^{\frac{1}{2}}\left(\frac{\int_{\Omega_{n}}\left|t_{n} v_{n}(x)\right| d x}{s_{n}^{\beta_{0}}}\right)^{-1} \\
\leq & 2 s_{n}^{-2 \alpha_{0}} t_{n} t \lambda_{1}^{-1}\left|\Omega_{n}\right|^{\frac{1}{2}}\left(\frac{\int_{\Omega_{n}}\left|t_{n} v_{n}(x)\right| d x}{s_{n}^{\beta_{0}}}\right)^{-1},
\end{aligned}
$$

where $\lambda_{1}$ is the first eigenvalue of $\Delta$ on $\Omega$ and $\left|\Omega_{n}\right|$ is the measure of $\Omega_{n}$, and by (4.17) and (4.20),

$$
\begin{aligned}
\left|\Omega_{n}\right| & =\int_{\Omega_{n}} d x \leq \int_{\Omega_{n}}\left|t_{n} v_{n}(x)\right| d x<2 t_{n} t \frac{s_{n}^{\beta_{0}}}{\left|t_{n}-t\right|} s_{n}^{1-\beta_{0}} \int_{\Omega_{n}}\left|d_{n}(x)\right| d x \\
& =2 s_{n}^{\frac{1}{2}-\alpha_{0}} t_{n} t D_{n}^{-1} \int_{\Omega_{n}}\left|d_{n}(x)\right| d x \leq 2 s_{n}^{\frac{1}{2}-\alpha_{0}} t_{n} t D_{n}^{-1} \lambda_{1}^{-1}\left|\Omega_{n}\right|^{\frac{1}{2}},
\end{aligned}
$$


i.e.,

$$
\left|\Omega_{n}\right| \leq s_{n}^{1-2 \alpha_{0}}
$$

Then,

i.e.,

$$
\frac{\left|t_{n}-t\right|}{s_{n}^{\beta_{0}}} \leq 2 s_{n}^{\frac{1}{2}-3 \alpha_{0}} t_{n} t\left(\frac{\int_{\Omega_{n}}\left|t_{n} v_{n}(x)\right| d x}{s_{n}^{\beta_{0}}}\right)^{-1}
$$

$$
\frac{\int_{\Omega_{n}}\left|t_{n} v_{n}(x)\right| d x}{s_{n}^{\beta_{0}}} \leq 2 s_{n}^{\frac{1}{2}-3 \alpha_{0}} t_{n} t\left(\frac{\left|t_{n}-t\right|}{s_{n}^{\beta_{0}}}\right)^{-1}=2 s_{n}^{\frac{1}{2}-3 \alpha_{0}} t_{n} t D_{n}^{-1} .
$$

Thus, since $\alpha_{0} \in\left(0, \frac{1}{6}\right]$, by (4.17), if $t>t_{n}$ and $n$ is large, then

$$
\int_{\Omega_{n}}\left|t_{n} v_{n}(x)\right| d x \leq D s_{n}^{\beta_{0}},
$$

where $D>0$ is a constant. By (4.16) and (4.21), if $t>t_{n}$ and $n$ is large, then

$$
\begin{aligned}
& \int_{\Omega_{n}}|t v(x)| d x \leq \int_{\Omega_{n}}\left|t\left(v(x)-s_{n} d_{n}(x)\right)\right| d x+\int_{\Omega_{n}}\left|t s_{n} d_{n}(x)\right| d x \\
\leq & t t_{n}^{-1} \sqrt{1+s_{n}^{2}} \int_{\Omega_{n}}\left|t_{n} v_{n}(x)\right| d x+t\left|\Omega_{n}\right| s_{n}\left(\int_{\Omega_{n}}\left|d_{n}(x)\right|^{2} d x\right)^{\frac{1}{2}} \\
\leq & t t_{n}^{-1} D \sqrt{1+s_{n}^{2}} s_{n}^{\beta_{0}}+t\left|\Omega_{n}\right| s_{n} \leq C s_{n}^{\beta_{0}},
\end{aligned}
$$

where $\left|\Omega_{n}\right|$ is the measure of $\Omega_{n}$ and $C>0$ is a constant. Hence, either there is $\left\{t_{n_{k}}\right\}$ such that $t<t_{n_{k}}$ or there is $\left\{t_{n_{k}}\right\}$ and a constant $C>0$ such that $t>t_{n_{k}}$ and

$$
\int_{\Omega_{n_{k}}}|t v(x)| d x \leq C s_{n_{k}}^{\beta_{0}}
$$

where $\Omega_{n_{k}}=C_{1}^{n_{k}} \cup C_{2}^{n_{k}} \cup B_{1}^{n_{k}}$.

(b) The conclusion can be proved in the same way as (a).

Lemma 4.12. Assume that $L=\{0\}, f(x, t)$ is in (4.2), $p$ is a peak selection of $J$ (4.1) defined on $S_{L^{\perp}}, p(w)=t_{w} w, t_{w}>0$ for $w \in S_{L^{\perp}}, p$ is continuous at $v \in S_{L^{\perp}}$ and $\left\{v_{n}\right\} \subset S_{L^{\perp}}$ satisfies $\left\|v_{n}-v\right\| \leq 1, v_{n} \rightarrow v$. Denote $p\left(v_{n}\right)=t_{n} v_{n}, t_{n}>0$ and $p(v)=t v, t>0 . A_{i}^{n}, B_{i}^{n}, C_{i}^{n}, i=1,2,3$, are defined in Lemma 4.10. Then,

(a)

$$
G_{0}^{n}+G_{1}^{n}+G_{2}^{n}+G_{3}^{n}=A_{n}
$$

where

$$
A_{n}=A_{n}^{+}+A_{n}^{0}+A_{n}^{-}
$$

with

$$
\begin{gathered}
A_{n}^{+}=c \alpha t_{n}^{\alpha} \int_{\Omega_{n}^{+}}|x|^{r} U^{n}(\alpha)(x) d x, A_{n}^{0}=c\left(\beta+k_{n}\right) t_{n}^{\beta} \int_{\Omega_{n}^{0}}|x|^{r} U^{n}(\beta)(x) d x, \\
A_{n}^{-}=c \beta t_{n}^{\beta} \int_{\Omega_{n}^{-}}|x|^{r} U^{n}(\beta)(x) d x, U^{n}(\gamma)=\left|v_{n}\right|^{\gamma}-|v|^{\gamma} \text { for } \gamma>0,
\end{gathered}
$$

and

$$
\Omega_{n}^{+}=A_{1}^{n} \cup B_{1}^{n} \cup C_{1}^{n}, \quad \Omega_{n}^{0}=A_{2}^{n} \cup B_{2}^{n} \cup C_{2}^{n}, \quad \Omega_{n}^{-}=A_{3}^{n} \cup B_{3}^{n} \cup C_{3}^{n},
$$

$k_{n} \in[0, \alpha-\beta]$,

$$
G_{0}^{n}=G_{0}^{n^{+}}+G_{0}^{n^{0}}+G_{0}^{n^{-}}-f^{n}
$$


with

$$
G_{0}^{n^{+}}=c f^{n}(\alpha) \int_{\Omega_{n}^{+}} \alpha|x|^{r}|v(x)|^{\alpha} d x, \quad G_{0}^{n^{0}}=c f^{n}(\beta) \int_{\Omega_{n}^{0}}\left(\beta+k_{n}\right)|x|^{r}|v(x)|^{\beta} d x
$$

and

$$
\begin{gathered}
G_{0}^{n^{-}}=c f^{n}(\beta) \int_{\Omega_{n}^{-}} \beta|x|^{r}|v(x)|^{\beta} d x, \quad f^{n}(\gamma)=t^{\gamma}-t_{n}^{\gamma} \text { for } \gamma>0, \\
G_{1}^{n}=I_{1}^{n}+I_{2}^{n}+I_{3}^{n}
\end{gathered}
$$

with

and

$$
\begin{gathered}
I_{1}^{n}=c \alpha \int_{A_{2}^{n}}|x|^{r}|t v(x)|^{\alpha} d x-c\left(\beta+k_{n}\right) \int_{A_{2}^{n}}|x|^{r}|t v(x)|^{\beta} d x \geq 0, \\
I_{2}^{n}=c \alpha \int_{A_{3}^{n}}|x|^{r}|t v(x)|^{\alpha} d x-c \beta \int_{A_{3}^{n}}|x|^{r}|t v(x)|^{\beta} d x \geq 0,
\end{gathered}
$$

$k \in[0, \alpha-\beta]$,

$$
I_{3}^{n}=c k \int_{B_{3}^{n}}|x|^{r}|t v(x)|^{\beta} d x \geq 0,
$$

with

$$
G_{2}^{n}=J_{1}^{n}+J_{2}^{n}+J_{3}^{n}
$$

$$
\begin{gathered}
J_{1}^{n}=c \beta \int_{C_{1}^{n}}|x|^{r}|t v(x)|^{\beta} d x-c \alpha \int_{C_{1}^{n}}|x|^{r}|t v(x)|^{\alpha} d x \\
J_{2}^{n}=-c k_{n} \int_{C_{2}^{n}}|t v(x)|^{\beta} d x
\end{gathered}
$$

and

$$
J_{3}^{n}=c(\beta+k) \int_{B_{1}^{n}}|x|^{r}|t v(x)|^{\beta} d x-c \alpha \int_{B_{1}^{n}}|x|^{r}|t v(x)|^{\alpha} d x,
$$

and

$$
G_{3}^{n}=c\left(k-k_{n}\right) \int_{B_{2}^{n}}|x|^{r}|t v(x)|^{\beta} d x
$$

(b) there is a constant $D>0$ such that, for every $\bar{\Omega} \subseteq \Omega \subset \mathbb{R}^{m}, \gamma>1$ for $m=1,2$ and $1<\gamma \leq \frac{2 m}{m-2}$ for $m \geq 3$,

$$
\left.\left|\int_{\bar{\Omega}}\right| x\right|^{r} U^{n}(\gamma)(x) d x \mid \leq D s_{n}
$$

as $n$ is large and there is a constant $\bar{D}>0$ such that

$$
\left|A_{n}\right| \leq \bar{D} s_{n}
$$

where $v_{n}=\frac{1}{\sqrt{1+s_{n}^{2}}}\left(v-s_{n} d_{n}\right),\left\|d_{n}\right\|=1,\left\langle d_{n}, v\right\rangle=0$ and $s_{n} \geq 0$.

Proof. (a) Since $p\left(v_{n}\right)=t_{n} v_{n}, t_{n}>0$ and $p(v)=t v, t>0$, there are $k, k_{n} \in$ $[0, \alpha-\beta]$ such that

$$
t^{2}-c \int_{\Omega^{+}} \alpha f(x) d x-c \int_{\Omega^{0}}(\beta+k) g(x) d x-c \int_{\Omega^{-}} \beta g(x) d x=0,
$$

where $f(x)=|x|^{r}|t v(x)|^{\alpha}, g(x)=|x|^{r}|t v(x)|^{\beta}, \Omega^{+}=\{x \in \Omega|| t v(x) \mid>1\}, \Omega^{0}=$ $\{x \in \Omega|| t v(x) \mid=1\}$ and $\Omega^{-}=\{x \in \Omega|| t v(x) \mid<1\}$, and

$$
t_{n}^{2}-c \int_{\Omega_{n}^{+}} \alpha f_{n}(x) d x-c \int_{\Omega_{n}^{0}}\left(\beta+k_{n}\right) g_{n}(x) d x-c \int_{\Omega_{n}^{-}} \beta g_{n}(x) d x=0,
$$


where $f_{n}(x)=|x|^{r}\left|t_{n} v_{n}(x)\right|^{\alpha}$ and $g_{n}(x)=|x|^{r}\left|t_{n} v_{n}(x)\right|^{\beta}$, i.e.,

$$
t_{n}^{2}-c \int_{\Omega_{n}^{+}} \alpha \bar{f}_{n}(x) d x-c \int_{\Omega_{n}^{0}}\left(\beta+k_{n}\right) \bar{g}_{n}(x) d x-c \int_{\Omega_{n}^{-}} \beta \bar{g}_{n}(x) d x=A_{n},
$$

where $\bar{f}_{n}(x)=|x|^{r}\left|t_{n} v(x)\right|^{\alpha}$ and $\bar{g}_{n}(x)=|x|^{r}\left|t_{n} v(x)\right|^{\beta}$.

By the first and third equality, after simple calculation, we can find that the conclusion is correct.

(b) Indeed, as $n$ is large,

$$
\begin{aligned}
& \left.\left|\int_{\bar{\Omega}}\right| x\right|^{r} U^{n}(\gamma)(x) d x|=| \int_{\bar{\Omega}}|x|^{r}\left|v_{n}(x)\right|^{\gamma} d x-\int_{\bar{\Omega}}|x|^{r}|v(x)|^{\gamma} d x \mid \\
= & \gamma\left(\phi\left(v_{n}, v, \eta\right)\right)^{\gamma-1}\left|\left(\int_{\bar{\Omega}}|x|^{r}\left|v_{n}(x)\right|^{\gamma} d x\right)^{\frac{1}{\gamma}}-\left(\int_{\bar{\Omega}}|x|^{r}|v(x)|^{\gamma} d x\right)^{\frac{1}{\gamma}}\right| \\
\leq & \gamma\left(\phi\left(v_{n}, v, \eta\right)\right)^{\gamma-1}\left(\int_{\bar{\Omega}}|x|^{r}\left|v_{n}(x)-v(x)\right|^{\gamma} d x\right)^{\frac{1}{\gamma}} \\
\leq & 2^{\gamma-1} \gamma\left(\int_{\bar{\Omega}}|x|^{r}|v(x)|^{\gamma} d x\right)^{\frac{\gamma-1}{\gamma}}\left(\int_{\bar{\Omega}}|x|^{r}\left|v_{n}(x)-v(x)\right|^{\gamma} d x\right)^{\frac{1}{\gamma}} \\
\leq & 2^{\gamma-1} \gamma\left(\int_{\Omega}|x|^{r}|v(x)|^{\gamma} d x\right)^{\frac{\gamma-1}{\gamma}}\left(\int_{\Omega}|x|^{r}\left|v_{n}(x)-v(x)\right|^{\gamma} d x\right)^{\frac{1}{\gamma}} \\
\leq & 2^{\gamma-1} M \gamma\left(\int_{\Omega}|v(x)|^{\gamma} d x\right)^{\frac{\gamma-1}{\gamma}}\left(\int_{\Omega}\left|v_{n}(x)-v(x)\right|^{\gamma} d x\right)^{\frac{1}{\gamma}} \\
\leq & 2^{\gamma-1} C^{\gamma} M \gamma\|v\|^{\gamma-1}\left\|v_{n}-v\right\| \leq(2 C)^{\gamma} M \gamma\|v\|^{\gamma-1} \frac{s_{n}}{\sqrt{1+s_{n}^{2}}}\left\|d_{n}\right\| \\
= & (2 C)^{\gamma} M \gamma\|v\|^{\gamma-1} \frac{s_{n}}{\sqrt{1+s_{n}^{2}}},
\end{aligned}
$$

where $\phi\left(v_{n}, v, \eta\right)=\eta\left(\int_{\bar{\Omega}}|x|^{r}\left|v_{n}(x)\right|^{\gamma} d x\right)^{\frac{1}{\gamma}}+(1-\eta)\left(\int_{\bar{\Omega}}|x|^{r}|v(x)|^{\gamma} d x\right)^{\frac{1}{\gamma}}, \eta \in[0,1]$,

$$
M=\sup _{x \in \Omega}|x|^{r},
$$

and $C>0$ is a constant, i.e.,

$$
\left.\left|\int_{\bar{\Omega}}\right| x\right|^{r} U^{n}(\gamma)(x) d x \mid \leq D s_{n}
$$

where $D>0$ is a constant. Hence, as $n$ is large,

$$
\left|A_{n}\right| \leq\left|A_{n}^{+}\right|+\left|A_{n}^{0}\right|+\left|A_{n}^{-}\right| \leq c\left(\alpha t_{n}^{\alpha_{1}}+\left(\beta+k_{n}\right) t_{n}^{\beta}+\beta t_{n}^{\beta}\right) D s_{n},
$$

i.e., there is a constant $\bar{D}>0$ such that $\left|A_{n}\right| \leq \bar{D} s_{n}$.

Lemma 4.13. Assume that $L=\{0\}, f(x, t)$ is in (4.2), $p$ is a peak selection of $J$ (4.1) defined on $S_{L^{\perp}}, p(w)=t_{w} w, t_{w}>0$ for $w \in S_{L^{\perp}}, p$ is continuous at $v \in S_{L^{\perp}}$ and $\left\{v_{n}\right\} \subset S_{L^{\perp}}$ satisfies $\left\|v_{n}-v\right\| \leq 1, v_{n} \rightarrow v$. Denote $p\left(v_{n}\right)=t_{n} v_{n}, t_{n}>0$ and $p(v)=t v, t>0 . A_{i}^{n}, B_{i}^{n}, C_{i}^{n}, i=1,2,3$, are defined in Lemma 4.10. Then,

(a)

$$
H_{0}^{n}+H_{1}^{n}+H_{2}^{n}+H_{3}^{n}=-B_{n}
$$

where

$$
B_{n}=B_{n}^{+}+B_{n}^{0}+B_{n}^{-}
$$


with

$$
\begin{gathered}
B_{n}^{+}=c \alpha t^{\alpha} \int_{\Omega^{+}}|x|^{r} U^{n}(\alpha)(x) d x, \quad B_{n}^{0}=c(\beta+k) t^{\beta} \int_{\Omega^{0}}|x|^{r} U^{n}(\beta)(x) d x, \\
B_{n}^{-}=c \beta t^{\beta} \int_{\Omega^{-}}|x|^{r} U^{n}(\beta)(x) d x, \quad U^{n}(\gamma)=\left|v_{n}\right|^{\gamma}-|v|^{\gamma} \text { for } \gamma>0,
\end{gathered}
$$

and

$$
\Omega^{+}=A_{1}^{n} \cup A_{2}^{n} \cup A_{3}^{n}, \quad \Omega^{0}=B_{1}^{n} \cup B_{2}^{n} \cup B_{3}^{n}, \Omega^{-}=C_{1}^{n} \cup C_{2}^{n} \cup C_{3}^{n},
$$

$k \in[0, \alpha-\beta]$,

$$
H_{0}^{n}=H_{0}^{n^{+}}+H_{0}^{n^{0}}+H_{0}^{n^{-}}-f^{n}(2)
$$

with

$$
H_{0}^{n^{+}}=c f^{n}(\alpha) \int_{\Omega^{+}} \alpha|x|^{r}\left|v_{n}(x)\right|^{\alpha} d x, \quad H_{0}^{n^{0}}=c f^{n}(\beta) \int_{\Omega^{0}}(\beta+k)|x|^{r}\left|v_{n}(x)\right|^{\beta} d x,
$$

and

$$
\begin{gathered}
H_{0}^{n^{-}}=c f^{n}(\beta) \int_{\Omega^{-}} \beta|x|^{r}\left|v_{n}(x)\right|^{\beta} d x, \quad f^{n}(\gamma)=t_{n}^{\gamma}-t^{\gamma} \text { for } \gamma>0, \\
H_{1}^{n}=I_{1}^{n}+I_{2}^{n}+I_{3}^{n}
\end{gathered}
$$

with

$$
\begin{gathered}
I_{1}^{n}=c \alpha \int_{C_{1}^{n}}|x|^{r}\left|t_{n} v_{n}(x)\right|^{\alpha} d x-c \beta \int_{C_{1}^{n}}|x|^{r}\left|t_{n} v_{n}(x)\right|^{\beta} d x \geq 0, \\
I_{2}^{n}=c k_{n} \int_{C_{2}^{n}}|x|^{r}\left|t_{n} v_{n}(x)\right|^{\beta} d x \geq 0,
\end{gathered}
$$

and

$$
I_{3}^{n}=c \alpha \int_{B_{1}^{n}}|x|^{r}\left|t_{n} v_{n}(x)\right|^{\alpha} d x-c(\beta+k) \int_{B_{1}^{n}}|x|^{r}\left|t_{n} v_{n}(x)\right|^{\beta} d x \geq 0,
$$

$k_{n} \in[0, \alpha-\beta]$,

$$
H_{2}^{n}=J_{1}^{n}+J_{2}^{n}+J_{3}^{n}
$$

with

$$
\begin{gathered}
J_{1}^{n}=c \beta \int_{A_{3}^{n}}|x|^{r}\left|t_{n} v_{n}(x)\right|^{\beta} d x-c \alpha \int_{A_{3}^{n}}|x|^{r}\left|t_{n} v_{n}(x)\right|^{\alpha} d x, \\
J_{2}^{n}=-c k \int_{B_{3}^{n}}|x|^{r}\left|t_{n} v_{n}(x)\right|^{\beta} d x
\end{gathered}
$$

and

$$
J_{3}^{n}=c\left(\beta+k_{n}\right) \int_{A_{2}^{n}}|x|^{r}\left|t_{n} v_{n}(x)\right|^{\beta} d x-c \alpha \int_{A_{2}^{n}}|x|^{r}\left|t_{n} v_{n}(x)\right|^{\alpha} d x,
$$

and

$$
H_{3}^{n}=c\left(k_{n}-k\right) \int_{B_{2}^{n}}|x|^{r}\left|t_{n} v_{n}(x)\right|^{\beta} d x,
$$

(b) there is a constant $\bar{D}>0$ such that

$$
\left|B_{n}\right| \leq \bar{D} s_{n}
$$

where $v_{n}=\frac{1}{\sqrt{1+s_{n}^{2}}}\left(v-s_{n} d_{n}\right),\left\|d_{n}\right\|=1,\left\langle d_{n}, v\right\rangle=0$ and $s_{n}>0$. 
Proof. (a) Since $p\left(v_{n}\right)=t_{n} v_{n}, t_{n}>0$ and $p(v)=t v, t>0$, there are $k, k_{n} \in$ $[0, \alpha-\beta]$ such that

$$
t^{2}-c \int_{\Omega^{+}} \alpha f(x) d x-c \int_{\Omega^{0}}(\beta+k) g(x) d x-c \int_{\Omega^{-}} \beta g(x) d x=0,
$$

where $f(x)=|x|^{r}|t v(x)|^{\alpha}$ and $g(x)=|x|^{r}|t v(x)|^{\beta}$, i.e.,

$$
t^{2}-c \int_{\Omega^{+}} \alpha \bar{f}_{n}(x) d x-c \int_{\Omega^{0}}(\beta+k) \bar{g}_{n}(x) d x-c \int_{\Omega^{-}} \beta \bar{g}_{n}(x) d x=-B_{n},
$$

where $\bar{f}_{n}(x)=|x|^{r}\left|t v_{n}(x)\right|^{\alpha}$ and $\bar{g}_{n}(x)=|x|^{r}\left|t v_{n}(x)\right|^{\beta}$, and

$$
t_{n}^{2}-c \int_{\Omega_{n}^{+}} \alpha f_{n}(x) d x-c \int_{\Omega_{n}^{0}}\left(\beta+k_{n}\right) g_{n}(x) d x-c \int_{\Omega_{n}^{-}} \beta g_{n}(x) d x=0,
$$

where $f_{n}(x)=|x|^{r}\left|t_{n} v_{n}(x)\right|^{\alpha}, g_{n}(x)=|x|^{r}\left|t_{n} v_{n}(x)\right|^{\beta}, \Omega_{n}^{+}=A_{1}^{n} \cup B_{1}^{n} \cup C_{1}^{n}, \Omega_{n}^{0}=$ $A_{2}^{n} \cup B_{2}^{n} \cup C_{2}^{n}$ and $\Omega_{n}^{-}=A_{3}^{n} \cup B_{3}^{n} \cup C_{3}^{n}$.

By the second and third equality, after simple computation, we should know that the conclusion is true.

(b) By (4.22), as $n$ is large,

$$
\left|B_{n}\right| \leq\left|B_{n}^{+}\right|+\left|B_{n}^{0}\right|+\left|B_{n}^{-}\right| \leq c\left(\alpha t^{\alpha_{1}}+(\beta+k) t^{\alpha_{2}}+\beta t^{\beta}\right) D s_{n},
$$

i.e., there is a constant $\bar{D}>0$ such that $\left|B_{n}\right| \leq \bar{D} s_{n}$.

Lemma 4.14. Assume that $L=\{0\}, f(x, t)$ is in (4.2), $p$ is a peak selection of $J$ (4.1) defined on $S_{L^{\perp}}, p(w)=t_{w} w, t_{w}>0$ for $w \in S_{L^{\perp}}, p$ is continuous at $v \in S_{L^{\perp}}$ and $\left\{v_{n}\right\} \subset S_{L^{\perp}}$ satisfies $\left\|v_{n}-v\right\| \leq 1, v_{n} \rightarrow v$. Denote $p\left(v_{n}\right)=t_{n} v_{n}, t_{n}>0$, $p(v)=t v, t>0$ and $\beta_{0}=\frac{1}{2}+\alpha_{0}, \alpha_{0} \in\left(0, \frac{1}{6}\right] . A_{i}^{n}, B_{i}^{n}, C_{i}^{n}, i=1,2,3$, are defined in Lemma 4.10. If $A_{2}^{n} \cup A_{3}^{n} \cup B_{3}^{n} \neq \emptyset$ and $t>t_{n}$ for $n=1,2, \ldots$, then, there is $a$ constant $L_{h}>0$ such that

$$
\left|t_{n}-t\right| \leq L_{h}\left\|v_{n}-v\right\|^{\beta_{0}} .
$$

Proof. Suppose that there is not a constant $L_{h}>0$ such that

$$
\left|t_{n}-t\right| \leq L_{h}\left\|v_{n}-v\right\|^{\beta_{0}} .
$$

Then, there is $\left\{v_{n_{l}}\right\}$ such that

$$
\lim _{l \rightarrow \infty} \frac{\left|t_{n_{l}}-t\right|}{\left\|v_{n_{l}}-v\right\|^{\beta_{0}}}=+\infty .
$$

Thus, similar to Lemma 4.10,

$$
\frac{\left|t_{n_{l}}-t\right|}{s_{n_{l}}^{\beta_{0}}} \rightarrow+\infty
$$

where $v_{n_{l}}=\frac{1}{\sqrt{1+s_{n_{l}}^{2}}}\left(v-s_{n_{l}} d_{n_{l}}\right),\left\|d_{n_{l}}\right\|=1,\left\langle d_{n_{l}}, v\right\rangle=0$ and $s_{n_{l}} \geq 0$.

First, by Lemma 4.11, there are following four cases. In these four cases, $\left\{n_{l^{\prime}}\right\}$ represents a subsequence of $\left\{n_{l}\right\}$.

The first case is there are $\left\{C_{1}^{n_{l^{\prime}}} \cup C_{2}^{n_{l^{\prime}}} \cup B_{1}^{n_{l^{\prime}}}\right\}$ and $\left\{B_{2}^{n_{l^{\prime}}}\right\}$ such that $C_{1}^{n_{l^{\prime}}} \cup C_{2}^{n_{l^{\prime}}} \cup$ $B_{1}^{n_{l^{\prime}}} \neq \emptyset, B_{2}^{n_{l^{\prime}}} \neq \emptyset$,

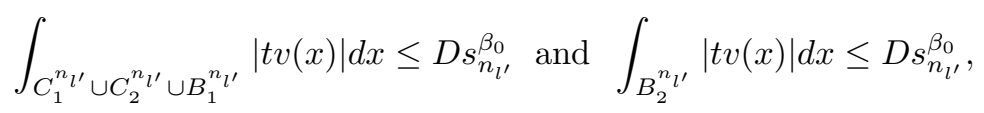

where $D>0$ is a constant. 
The second case is there are $\left\{C_{1}^{n_{l^{\prime}}} \cup C_{2}^{n_{l^{\prime}}} \cup B_{1}^{n_{l^{\prime}}}\right\}$ and $\left\{B_{2}^{n_{l^{\prime}}}\right\}$ such that $C_{1}^{n_{l^{\prime}}} \cup$ $C_{2}^{n_{l^{\prime}}} \cup B_{1}^{n_{l^{\prime}}} \neq \emptyset, B_{2}^{n_{l^{\prime}}}=\emptyset$ and

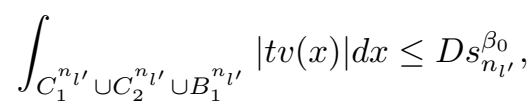

where $D>0$ is a constant.

The third case is there are $\left\{C_{1}^{n_{l^{\prime}}} \cup C_{2}^{n_{l^{\prime}}} \cup B_{1}^{n_{l^{\prime}}}\right\}$ and $\left\{B_{2}^{n_{l^{\prime}}}\right\}$ such that $C_{1}^{n_{l^{\prime}}} \cup$ $C_{2}^{n_{l^{\prime}}} \cup B_{1}^{n_{l^{\prime}}}=\emptyset, B_{2}^{n_{l^{\prime}}} \neq \emptyset$ and

$$
\int_{B_{2}^{n_{l^{\prime}}}}|t v(x)| d x \leq D s_{n_{l^{\prime}}}^{\beta_{0}}
$$

where $D>0$ is a constant.

The fourth case is there are $\left\{C_{1}^{n_{l^{\prime}}} \cup C_{2}^{n_{l^{\prime}}} \cup B_{1}^{n_{l^{\prime}}}\right\}$ and $\left\{B_{2}^{n_{l^{\prime}}}\right\}$ such that $C_{1}^{n_{l^{\prime}}} \cup$ $C_{2}^{n_{l^{\prime}}} \cup B_{1}^{n_{l^{\prime}}}=\emptyset$ and $B_{2}^{n_{l^{\prime}}}=\emptyset$.

We only discuss the first case and the remaining three cases can be handled in a similar way. By (4.24),

$$
\begin{gathered}
\left|J_{1}^{n_{l^{\prime}}}\right|=\left.\left|c \beta \int_{C_{1}^{n_{l^{\prime}}}}\right| x\right|^{r}|t v(x)|^{\beta} d x-c \alpha \int_{C_{1}^{n_{l^{\prime}}}}|x|^{r}|t v(x)|^{\alpha} d x \mid \\
\leq c \beta M \int_{C_{1}^{n_{l^{\prime}}}}|t v(x)| d x+c \alpha M \int_{C_{1}^{n_{l^{\prime}}}}|t v(x)| d x \leq c(\beta+\alpha) M D s_{n_{l^{\prime}}}^{\beta_{0}}, \\
\left|J_{2}^{n_{l^{\prime}}}\right|=\left.\left|-c k_{n_{l^{\prime}}} \int_{C_{2}^{n_{l^{\prime}}}}\right| x\right|^{r}|t v(x)|^{\beta} d x\left|\leq c k_{n_{l^{\prime}}} M \int_{C_{2}^{n_{l^{\prime}}}}\right| t v(x) \mid d x \leq c k_{n_{l^{\prime}}} M D s_{n_{l^{\prime}}}^{\beta_{0}},
\end{gathered}
$$

and

$$
\begin{aligned}
& \left|J_{3}^{n_{l^{\prime}}}\right|=\left.\left|c(\beta+k) \int_{B_{1}^{n_{l^{\prime}}}}\right| x\right|^{r}|t v(x)|^{\beta} d x-c \alpha \int_{B_{1}^{n_{l^{\prime}}}}|x|^{r}|t v(x)|^{\alpha} d x \mid \\
\leq & c(\beta+k) M \int_{B_{1}^{n_{l^{\prime}}}}|t v(x)| d x+c \alpha M \int_{B_{1}^{n_{l^{\prime}}}}|t v(x)| d x \leq c(\beta+\alpha+k) M D s_{n_{l^{\prime}}}^{\beta_{0}},
\end{aligned}
$$

where $M=\sup _{x \in \Omega}|x|^{r}$. These three inequalities mean that

$$
\left|G_{2}^{n_{l^{\prime}}}\right| \leq\left|J_{1}^{n_{l^{\prime}}}\right|+\left|J_{2}^{n_{l^{\prime}}}\right|+\left|J_{3}^{n_{l^{\prime}}}\right| \leq c\left(2 \alpha+2 \beta+k_{n_{l^{\prime}}}+k\right) M D s_{n_{l^{\prime}}}^{\beta_{0}} .
$$

By (4.24),

$$
\left|G_{3}^{n_{l^{\prime}}}\right|=\left.\left|c\left(k-k_{n_{l^{\prime}}}\right) \int_{B_{2}^{n_{l^{\prime}}}}\right| x\right|^{r}|t v(x)|^{\beta} d x|\leq c| k-k_{n_{l^{\prime}}}\left|M \int_{B_{2}^{n_{l^{\prime}}}}\right| t v(x) \mid d x
$$

i.e.,

$$
\left|G_{3}^{n_{l^{\prime}}}\right| \leq c\left|k-k_{n_{l^{\prime}}}\right| M D s_{n_{l^{\prime}}}^{\beta_{0}} .
$$

Then, by (4.25), 4.26) and Lemma 4.12,

$$
\begin{aligned}
& G_{0}^{n_{l^{\prime}}} \leq G_{0}^{n_{l^{\prime}}}+G_{1}^{n_{l^{\prime}}}=A_{n_{l^{\prime}}}-G_{2}^{n_{l^{\prime}}}-G_{3}^{n_{l^{\prime}}} \leq\left|A_{n_{l^{\prime}}}\right|+\left|G_{2}^{n_{l^{\prime}}}\right|+\left|G_{3}^{n_{l^{\prime}}}\right| \\
\leq & \bar{D} s_{n_{l^{\prime}}}+c\left(2 \alpha+2 \beta+k_{n_{l^{\prime}}}+k\right) M D s_{n_{l^{\prime}}}^{\beta_{0}}+c\left|k-k_{n_{l^{\prime}}}\right| M D s_{n_{l^{\prime}}}^{\beta_{0}} \leq \bar{C} s_{n_{l^{\prime}}}^{\beta_{0}},
\end{aligned}
$$

where $\bar{C}, \bar{D}>0$ are two constants, i.e.,

$$
t-t_{n_{l^{\prime}}} \leq \frac{\bar{C}}{G^{n_{l^{\prime}}}} s_{n_{l^{\prime}}}^{\beta_{0}},
$$


where $G^{n_{l^{\prime}}}=G_{n_{l^{\prime}}}^{+}+G_{n_{l^{\prime}}}^{0}+G_{n_{l^{\prime}}}^{-}-\left(t+t_{n_{l^{\prime}}}\right)$ with

$$
\begin{gathered}
G_{n_{l^{\prime}}}^{+}=c \alpha\left(\eta_{1} t+\left(1-\eta_{1}\right) t_{n_{l^{\prime}}}\right)^{\alpha-1} \int_{\Omega_{n_{l^{\prime}}}^{+}} \alpha|x|^{r}|v(x)|^{\alpha} d x, \\
G_{n_{l^{\prime}}}^{0}=c \beta\left(\eta_{2} t+\left(1-\eta_{2}\right) t_{n_{l^{\prime}}}\right)^{\beta-1} \int_{\Omega_{n_{l^{\prime}}}^{0}}\left(\beta+k_{n_{l^{\prime}}}\right)|x|^{r}|v(x)|^{\beta} d x,
\end{gathered}
$$

and

$$
G_{n_{l^{\prime}}}^{-}=c \beta\left(\eta_{2} t+\left(1-\eta_{2}\right) t_{n_{l^{\prime}}}\right)^{\beta-1} \int_{\Omega_{n_{l^{\prime}}^{-}}^{-}} \beta|x|^{r}|v(x)|^{\beta} d x,
$$

$\eta_{1}, \eta_{2} \in[0,1]$. On the other hand, in the verification of Lemma 4.12, we have

$$
\left.\left|\int_{\bar{\Omega}}\right| x\right|^{r} U^{n}(\gamma)(x) d x \mid \leq 2^{\gamma-1} C^{\gamma} M \gamma\|v\|^{\gamma-1}\left\|v_{n}-v\right\|
$$

for $\bar{\Omega} \subseteq \Omega, \gamma>1$ for $m=1,2$ and $1<\gamma \leq \frac{2 m}{m-2}$ for $m \geq 3$, as $n$ is large, where $U^{n}(\gamma)=\left|v_{n}\right|^{\gamma}-|v|^{\gamma}$ and $C>0$ is a constant. Since $v_{n_{l^{\prime}}} \rightarrow v$, (4.28) means, $\forall \epsilon>0$,

$$
\left.\left|\int_{\hat{\Omega}}\right| x\right|^{r}\left|v_{n_{l^{\prime}}}(x)\right|^{\gamma} d x-\int_{\hat{\Omega}}|x|^{r}|v(x)|^{\gamma} d x \mid \leq \epsilon,
$$

for $(\gamma, \hat{\Omega})=\left(\alpha, \Omega_{n_{l^{\prime}}}^{+}\right),\left(\beta, \Omega_{n_{l^{\prime}}}^{0}\right),\left(\beta, \Omega_{n_{l^{\prime}}}^{-}\right)$, as $n_{l^{\prime}}$ is large. By this inequality, $\forall \zeta>0$,

$$
\left|G^{n_{l^{\prime}}}-\bar{G}^{n_{l^{\prime}}}\right| \leq \zeta
$$

as $n_{l^{\prime}}$ is large, where $\bar{G}^{n_{l^{\prime}}}=\bar{G}_{n_{l^{\prime}}}^{+}+\bar{G}_{n_{l^{\prime}}}^{0}+\bar{G}_{n_{l^{\prime}}}^{-}-\left(t+t_{n_{l^{\prime}}}\right)$ with

$$
\begin{gathered}
\bar{G}_{n_{l^{\prime}}}^{+}=c \alpha\left(\eta_{1} t+\left(1-\eta_{1}\right) t_{n_{l^{\prime}}}\right)^{\alpha-1} \int_{\Omega_{n_{l^{\prime}}}^{+}} \alpha|x|^{r}\left|v_{n_{l^{\prime}}}(x)\right|^{\alpha} d x, \\
\bar{G}_{n_{l^{\prime}}}^{0}=c \beta\left(\eta_{2} t+\left(1-\eta_{2}\right) t_{n_{l^{\prime}}}\right)^{\beta-1} \int_{\Omega_{n_{l^{\prime}}}^{0}}\left(\beta+k_{n_{l^{\prime}}}\right)|x|^{r}\left|v_{n_{l^{\prime}}}(x)\right|^{\beta} d x,
\end{gathered}
$$

and

$$
\bar{G}_{n_{l^{\prime}}}^{-}=c \beta\left(\eta_{2} t+\left(1-\eta_{2}\right) t_{n_{l^{\prime}}}\right)^{\beta-1} \int_{\Omega_{n_{l^{\prime}}}^{-}} \beta|x|^{r}\left|v_{n_{l^{\prime}}}(x)\right|^{\beta} d x .
$$

Since $p\left(v_{n}\right)=t_{n} v_{n}, t_{n}>0$, we have

$$
t_{n}^{2}-c \int_{\Omega_{n}^{+}} \alpha f_{n}(x) d x-c \int_{\Omega_{n}^{0}}\left(\beta+k_{n}\right) g_{n}(x) d x-c \int_{\Omega_{n}^{-}} \beta g_{n}(x) d x=0,
$$

where $f_{n}(x)=|x|^{r}\left|t_{n} v_{n}(x)\right|^{\alpha}$ and $g_{n}(x)=|x|^{r}\left|t_{n} v_{n}(x)\right|^{\beta}$. Thus, for large $n_{l^{\prime}}$,

$$
\bar{G}^{n_{l^{\prime}}} \geq\left(\beta-\frac{\beta-2}{4}\right) t_{n_{l^{\prime}}}-\left(2+\frac{\beta-2}{4}\right) t_{n_{l^{\prime}}}=\frac{\beta-2}{2} t_{n_{l^{\prime}}} \geq \frac{\beta-2}{4} t .
$$

By (4.29) and (4.30), if we set $\zeta=\frac{\beta-2}{8} t$, then, for large $n_{l^{\prime}}$,

$$
G^{n_{l^{\prime}}}>\frac{\beta-2}{8} t
$$

By (4.27) and (4.31), for large $n_{l^{\prime}}$,

$$
\frac{\left|t_{n_{l^{\prime}}}-t\right|}{s_{n_{l^{\prime}}}^{\beta_{0}}}=\frac{t-t_{n_{l^{\prime}}}}{s_{n_{l^{\prime}}}^{\beta_{0}}}<\frac{8 \bar{C}}{(\beta-2) t} .
$$

This is a contradiction to (4.23). Hence, the conclusion is true. 
Lemma 4.15. Assume that $L=\{0\}, f(x, t)$ is in (4.2), $p$ is a peak selection of $J$ (4.1) defined on $S_{L^{\perp}}, p(w)=t_{w} w, t_{w}>0$ for $w \in S_{L^{\perp}}, p$ is continuous at $v \in S_{L^{\perp}}$ and $\left\{v_{n}\right\} \subset S_{L \perp}$ satisfies $\left\|v_{n}-v\right\| \leq 1, v_{n} \rightarrow v$. Denote $p\left(v_{n}\right)=t_{n} v_{n}, t_{n}>0$, $p(v)=t v, t>0$ and $\beta_{0}=\frac{1}{2}+\alpha_{0}, \alpha_{0} \in\left(0, \frac{1}{6}\right] . A_{i}^{n}, B_{i}^{n}, C_{i}^{n}, i=1,2,3$, are defined in Lemma 4.10. If $A_{2}^{n} \cup A_{3}^{n} \cup B_{3}^{n} \neq \emptyset, t<t_{n}$ and

$$
\int_{\Omega_{n}}\left|t_{n} v_{n}(x)\right| d x \leq D s_{n}^{\beta_{0}}
$$

for $n=1,2, \ldots$, where $\Omega_{n}=A_{2}^{n} \cup A_{3}^{n} \cup B_{3}^{n}, v_{n}=\frac{1}{\sqrt{1+s_{n}^{2}}}\left(v-s_{n} d_{n}\right),\left\|d_{n}\right\|=1$, $\left\langle d_{n}, v\right\rangle=0, s_{n} \geq 0$ and $D>0$ is a constant, then, there is a constant $L_{h}>0$ such that

$$
\left|t_{n}-t\right| \leq L_{h}\left\|v_{n}-v\right\|^{\beta_{0}} .
$$

Proof. Suppose that there is not a constant $L_{h}>0$ such that

$$
\left|t_{n}-t\right| \leq L_{h}\left\|v_{n}-v\right\|^{\beta_{0}} .
$$

Then, similar to Lemma 4.14, there is $\left\{t_{n_{l}}\right\}$ such that

$$
\frac{\left|t_{n_{l}}-t\right|}{s_{n_{l}}^{\beta_{0}}} \rightarrow+\infty \text {. }
$$

By Lemma 4.10, there are the following two cases. In these two cases, $\left\{n_{l^{\prime}}\right\}$ represents a subsequence of $\left\{n_{l}\right\}$.

The first case is there is $\left\{B_{2}^{n_{l^{\prime}}}\right\}$ such that $B_{2}^{n_{l^{\prime}}} \neq \emptyset$,

$$
\int_{B_{2}^{n_{l^{\prime}}}}\left|t_{n_{l^{\prime}}} v_{n_{l^{\prime}}}(x)\right| d x \leq \hat{D} s_{n_{l^{\prime}}}^{\beta_{0}}
$$

where $\hat{D}>0$ is a constant.

The second case is there is $\left\{B_{2}^{n_{l^{\prime}}}\right\}$ such that $B_{2}^{n_{l^{\prime}}}=\emptyset$.

We only work on the first case and the second case can be discussed in a similar way.

By (4.32),

$$
\begin{gathered}
\left|J_{1}^{n_{l^{\prime}}}\right|=\left.\left|c \beta \int_{A_{3}^{n_{l^{\prime}}}}\right| x\right|^{r}\left|t_{n_{l^{\prime}}} v_{n_{l^{\prime}}}(x)\right|^{\beta} d x-c \alpha \int_{A_{3}^{n_{l^{\prime}}}}|x|^{r}\left|t_{n_{l^{\prime}}} v_{n_{l^{\prime}}}(x)\right|^{\alpha} d x \mid \\
\leq c \beta M \int_{A_{3}^{n_{l^{\prime}}}}\left|t_{n_{l^{\prime}}} v_{n_{l^{\prime}}}(x)\right| d x+c \alpha M \int_{A_{3}^{n_{l^{\prime}}}}\left|t_{n_{l^{\prime}}} v_{n_{l^{\prime}}}(x)\right| d x \leq c(\beta+\alpha) M D s_{n_{l^{\prime}}}^{\beta_{0}}, \\
\left|J_{2}^{n_{l^{\prime}}}\right|=\left.\left|c k \int_{B_{3}^{n_{l^{\prime}}}}\right| x\right|^{r}\left|t_{n_{l^{\prime}}} v_{n_{l^{\prime}}}(x)\right|^{\beta} d x\left|\leq c k M \int_{B_{3}^{n_{l^{\prime}}}}\right| t_{n_{l^{\prime}}} v_{n_{l^{\prime}}}(x) \mid d x \leq c k M D s_{n_{l^{\prime}}}^{\beta_{0}},
\end{gathered}
$$

and

$$
\begin{aligned}
& \left|J_{3}^{n_{l^{\prime}}}\right|=\left.\left|c\left(\beta+k_{n_{l^{\prime}}}\right) \int_{A_{2}^{n_{l^{\prime}}}}\right| x\right|^{r}\left|t_{n_{l^{\prime}}} v_{n_{l^{\prime}}}(x)\right|^{\beta} d x-c \alpha \int_{A_{2}^{n_{l^{\prime}}}}|x|^{r}\left|t_{n_{l^{\prime}}} v_{n_{l^{\prime}}}(x)\right|^{\alpha} d x \mid \\
\leq & c\left(\beta+k_{n_{l^{\prime}}}\right) M \int_{A_{2}^{n_{l^{\prime}}}}\left|t_{n_{l^{\prime}}} v_{n_{l^{\prime}}}(x)\right| d x+c \alpha M \int_{A_{2}^{n_{l^{\prime}}}}\left|t_{n_{l^{\prime}}} v_{n_{l^{\prime}}}(x)\right| d x \\
\leq & c\left(\beta+\alpha+k_{n_{l^{\prime}}}\right) M D s_{n_{l^{\prime}}}^{\beta_{0}},
\end{aligned}
$$

where $M=\sup _{x \in \Omega}|x|^{r}$. These three inequalities mean that

$$
\left|H_{2}^{n_{l^{\prime}}}\right| \leq\left|J_{1}^{n_{l^{\prime}}}\right|+\left|J_{2}^{n_{l^{\prime}}}\right|+\left|J_{2}^{n_{l^{\prime}}}\right| \leq c\left(2 \beta+2 \alpha+k_{n_{l^{\prime}}}+k\right) M D s_{n_{l^{\prime}}}^{\beta_{0}} .
$$


By (4.34),

$$
\left|H_{3}^{n_{l^{\prime}}}\right|=\left.\left|c\left(k_{n_{l^{\prime}}}-k\right) \int_{B_{2}^{n_{l^{\prime}}}}\right| x\right|^{r}\left|t_{n_{l^{\prime}}} v_{n_{l^{\prime}}}(x)\right|^{\beta} d x|\leq c| k_{n_{l^{\prime}}}-k\left|M \int_{B_{2}^{n_{l^{\prime}}}}\right| t_{n_{l^{\prime}}} v_{n_{l^{\prime}}}(x) \mid d x
$$
i.e.,

$$
\left|H_{3}^{n_{l^{\prime}}}\right| \leq c\left|k-k_{n_{l^{\prime}}}\right| M \hat{D} s_{n_{l^{\prime}}}^{\beta_{0}}
$$

By (4.35), 4.36) and Lemma 4.13.

$$
\begin{aligned}
& H_{0}^{n_{l^{\prime}}} \leq H_{0}^{n_{l^{\prime}}}+H_{1}^{n_{l^{\prime}}}=-B_{n_{l^{\prime}}}-H_{2}^{n_{l^{\prime}}}-H_{3}^{n_{l^{\prime}}} \leq\left|B_{n_{l^{\prime}}}\right|+\left|H_{2}^{n_{l^{\prime}}}\right|+\left|H_{3}^{n_{l^{\prime}}}\right| \\
& \leq \quad \bar{D} s_{n_{l^{\prime}}}+c\left(2 \alpha+2 \beta+k_{n_{l^{\prime}}}+k\right) M D s_{n_{l^{\prime}}}^{\beta_{0}}+c\left|k-k_{n_{l^{\prime}}}\right| M \hat{D}_{n_{l^{\prime}}}^{\beta_{0}} \leq \bar{C} s_{n_{l^{\prime}}}^{\beta_{0}},
\end{aligned}
$$

where $\bar{C}, \bar{D}>0$ is a constant, i.e.,

$$
t_{n_{l^{\prime}}}-t \leq \frac{\bar{C}}{H^{n_{l^{\prime}}}} s_{n_{l^{\prime}}}^{\beta_{0}},
$$

where $H^{n_{l^{\prime}}}=H_{n_{l^{\prime}}}^{+}+H_{n_{l^{\prime}}}^{0}+H_{n_{l^{\prime}}}^{-}-\left(t+t_{n_{l^{\prime}}}\right)$ with

$$
\begin{gathered}
H_{n_{l^{\prime}}}^{+}=c \alpha\left(\eta_{1} t+\left(1-\eta_{1}\right) t_{n_{l^{\prime}}}\right)^{\alpha-1} \int_{\Omega^{+}} \alpha|x|^{r}\left|v_{n_{l^{\prime}}}(x)\right| \alpha d x, \\
H_{n_{l^{\prime}}}^{0}=c \beta\left(\eta_{2} t+\left(1-\eta_{2}\right) t_{n_{l^{\prime}}}\right)^{\beta-1} \int_{\Omega^{0}}(\beta+k)|x|^{r}\left|v_{n_{l^{\prime}}}(x)\right|^{\beta} d x,
\end{gathered}
$$

and

$$
H_{n_{l^{\prime}}}^{-}=c \beta\left(\eta_{2} t+\left(1-\eta_{2}\right) t_{n_{l^{\prime}}}\right)^{\beta-1} \int_{\Omega^{-}} \beta|x|^{r}\left|v_{n_{l^{\prime}}}(x)\right|^{\beta} d x,
$$

$\eta_{1}, \eta_{2} \in[0,1]$. Similar to Lemma 4.14, since $v_{n_{l^{\prime}}} \rightarrow v$, by (4.28), we have, $\forall \epsilon>0$,

$$
\left.\left|\int_{\hat{\Omega}}\right| x\right|^{r}\left|v_{n_{l^{\prime}}}(x)\right|^{\gamma} d x-\int_{\hat{\Omega}}|x|^{r}|v(x)|^{\gamma} d x \mid \leq \epsilon,
$$

where $(\gamma, \hat{\Omega})=\left(\alpha, \Omega^{+}\right),\left(\beta, \Omega^{0}\right),\left(\beta, \Omega^{-}\right)$, as $n_{l^{\prime}}$ is large. By this inequality, $\forall \zeta>0$,

$$
\left|H^{n_{l^{\prime}}}-\bar{H}^{n_{l^{\prime}}}\right| \leq \zeta,
$$

as $n_{l^{\prime}}$ is large, where $\bar{H}^{n_{l^{\prime}}}=\bar{H}_{n_{l^{\prime}}}^{+}+\bar{H}_{n_{l^{\prime}}}^{0}+\bar{H}_{n_{l^{\prime}}}^{-}-\left(t+t_{n_{l^{\prime}}}\right)$.

$$
\begin{gathered}
\bar{H}_{n_{l^{\prime}}}^{+}=c \alpha\left(\eta_{1} t+\left(1-\eta_{1}\right) t_{n_{l^{\prime}}}\right)^{\alpha-1} \int_{\Omega^{+}} \alpha_{1}|x|^{r}|v(x)|^{\alpha} d x, \\
\bar{H}_{n_{l^{\prime}}}^{0}=c \beta\left(\eta_{2} t+\left(1-\eta_{2}\right) t_{n_{l^{\prime}}}\right)^{\beta-1} \int_{\Omega^{0}}(\beta+k)|x|^{r}|v(x)|^{\beta} d x,
\end{gathered}
$$

and

Since $p(v)=t v, t>0$,

$$
\bar{H}_{n_{l^{\prime}}}^{-}=c \beta\left(\eta_{2} t+\left(1-\eta_{2}\right) t_{n_{l^{\prime}}}\right)^{\beta-1} \int_{\Omega^{-}} \beta|x|^{r}|v(x)|^{\beta} d x .
$$

$$
t^{2}-c \int_{\Omega^{+}} \alpha|t v(x)|^{\alpha} d x-c \int_{\Omega^{0}}(\beta+k)|t v(x)|^{\beta} d x-c \int_{\Omega^{-}} \beta|t v(x)|^{\beta} d x=0 .
$$

Then, for large $n_{l^{\prime}}$,

$$
\bar{H}^{n_{l^{\prime}}} \geq\left(\beta-\frac{\beta-2}{4}\right) t-\left(2+\frac{\beta-2}{4}\right) t=\frac{\beta-2}{2} t .
$$

By (4.38) and (4.39), if we set $\zeta=\frac{\beta-2}{4} t$, then, for large $n_{l^{\prime}}$,

$$
H^{n_{l^{\prime}}}>\frac{\beta-2}{4} t \text {. }
$$


By (4.37) and (4.40), for large $n_{l^{\prime}}$,

$$
\frac{\left|t_{n_{l^{\prime}}}-t\right|}{s_{n_{l^{\prime}}}^{\beta_{0}}}=\frac{t_{n_{l^{\prime}}}-t}{s_{n_{l^{\prime}}}^{\beta_{0}}}<\frac{4 \bar{C}}{(\beta-2) t} .
$$

This is a contradiction to (4.33). Hence, the conclusion is correct.

Lemma 4.16. Assume that $L=\{0\}, f(x, t)$ is in (4.2), $p$ is a peak selection of $J$ (4.1) defined on $S_{L^{\perp}}, p(w)=t_{w} w, t_{w}>0$ for $w \in S_{L^{\perp}}, p$ is continuous at $v \in S_{L^{\perp}}$ and $\left\{v_{n}\right\} \subset S_{L^{\perp}}$ satisfies $\left\|v_{n}-v\right\| \leq 1, v_{n} \rightarrow v$. Denote $p\left(v_{n}\right)=t_{n} v_{n}, t_{n}>0$, $p(v)=t v, t>0$ and $\beta_{0}=\frac{1}{2}+\alpha_{0}, \alpha_{0} \in\left(0, \frac{1}{6}\right] . A_{i}^{n}, B_{i}^{n}, C_{i}^{n}, i=1,2,3$, are defined in Lemma 4.10. If $A_{2}^{n} \cup A_{3}^{n} \cup B_{3}^{n}=\emptyset$ for $n=1,2, \ldots$, then, there is a constant $L_{h}>0$ such that

$$
\left|t_{n}-t\right| \leq L_{h}\left\|v_{n}-v\right\|^{\beta_{0}}
$$

Proof. By Lemma 2.12, denote $v_{n}=\frac{1}{\sqrt{1+s_{n}^{2}}}\left(v-s_{n} d_{n}\right),\left\|d_{n}\right\|=1,\left\langle d_{n}, v\right\rangle=0$ and $s_{n} \geq 0$. Suppose that there is not a constant $L_{h}>0$ such that

$$
\left|t_{n}-t\right| \leq L_{h}\left\|v_{n}-v\right\|^{\beta_{0}} .
$$

Then, similar to Lemma 4.14, there is $\left\{t_{n_{l}}\right\}$ such that

$$
\frac{\left|t_{n_{l}}-t\right|}{s_{n_{l}}^{\beta_{0}}} \rightarrow+\infty .
$$

Then, by Lemma 4.10 and Lemma 4.11, there are the following six cases. In these six cases, $\left\{n_{l^{\prime}}\right\}$ represents a subsequence of $\left\{n_{l}\right\}$.

The first case is there are $\left\{t_{n_{l^{\prime}}}\right\}$ and $\left\{B_{2}^{n_{l^{\prime}}}\right\}$ such that $t<t_{n_{l^{\prime}}}, B_{2}^{n_{l^{\prime}}} \neq \emptyset$ and

$$
\int_{B_{2}^{n_{l^{\prime}}}}\left|t_{n_{l^{\prime}}} v_{n_{l^{\prime}}}(x)\right| d x \leq D s_{n_{l^{\prime}}}^{\beta_{0}}
$$

where $D>0$ is a constant.

The second case is there are $\left\{t_{n_{l^{\prime}}}\right\}$ and $\left\{B_{2}^{n_{l^{\prime}}}\right\}$ such that $t<t_{n_{l^{\prime}}}$ and $B_{2}^{n_{l^{\prime}}}=\emptyset$.

The third case is there are $\left\{C_{1}^{n_{l^{\prime}}} \cup C_{2}^{n_{l^{\prime}}} \cup B_{1}^{n_{l^{\prime}}}\right\},\left\{t_{n_{l^{\prime}}}\right\}$ and $\left\{B_{2}^{n_{l^{\prime}}}\right\}$ such that $C_{1}^{n_{l^{\prime}}} \cup C_{2}^{n_{l^{\prime}}} \cup B_{1}^{n_{l^{\prime}}} \neq \emptyset, t>t_{n_{l^{\prime}}}, B_{2}^{n_{l^{\prime}}} \neq \emptyset$ and

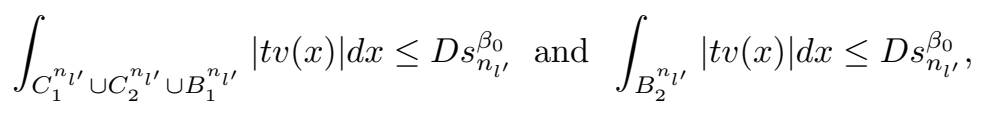

where $D>0$ is a constant.

The fourth case is there are $\left\{C_{1}^{n_{l^{\prime}}} \cup C_{2}^{n_{l^{\prime}}} \cup B_{1}^{n_{l^{\prime}}}\right\},\left\{t_{n_{l^{\prime}}}\right\}$ and $\left\{B_{2}^{n_{l^{\prime}}}\right\}$ such that $C_{1}^{n_{l^{\prime}}} \cup C_{2}^{n_{l^{\prime}}} \cup B_{1}^{n_{l^{\prime}}} \neq \emptyset, t>t_{n_{l^{\prime}}}, B_{2}^{n_{l^{\prime}}}=\emptyset$ and

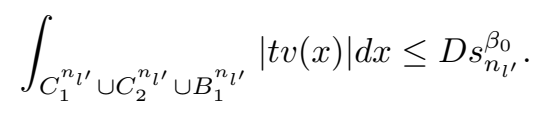

The fifth case is there are $\left\{C_{1}^{n_{l^{\prime}}} \cup C_{2}^{n_{l^{\prime}}} \cup B_{1}^{n_{l^{\prime}}}\right\},\left\{t_{n_{l^{\prime}}}\right\}$ and $\left\{B_{2}^{n_{l^{\prime}}}\right\}$ such that $C_{1}^{n_{l^{\prime}}} \cup C_{2}^{n_{l^{\prime}}} \cup B_{1}^{n_{l^{\prime}}}=\emptyset, t>t_{n_{l^{\prime}}}, B_{2}^{n_{l^{\prime}}} \neq \emptyset$ and

$$
\int_{B_{2}^{n_{l^{\prime}}}}|t v(x)| d x \leq D s_{n_{l^{\prime}}}^{\beta_{0}},
$$

where $D>0$ is a constant. 
The sixth case is there are $\left\{C_{1}^{n_{l^{\prime}}} \cup C_{2}^{n_{l^{\prime}}} \cup B_{1}^{n_{l^{\prime}}}\right\},\left\{t_{n_{l^{\prime}}}\right\}$ and $\left\{B_{2}^{n_{l^{\prime}}}\right\}$ such that

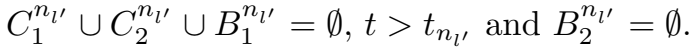

The first and second cases can be dealt with similarly to Lemma 4.15 and the third, fourth, fifth and sixth cases can be handled similarly to Lemma 4.14.

By Lemma 4.10-Lemma 4.16, we can proof the following theorem.

Theorem 4.17. Assume that $L=\{0\}, f(x, t)$ is in (4.2), $p$ is a peak selection of $J$ (4.10 defined on $S_{L^{\perp}}, p(w)=t_{w} w, t_{w}>0$ for $w \in S_{L^{\perp}}$ and $p$ is continuous at $v \in S_{L^{\perp}}$. Denote $p(v)=t v, t>0$. Then, there is a constant $L_{h}>0$ and a neighborhood $\mathcal{N}(v)$ of $v$ on $S_{L^{\perp}}$ such that, $\forall u \in \mathcal{N}(v)$,

$$
\|p(u)-p(v)\| \leq L_{h}\|u-v\|^{\beta_{0}},
$$

where $\beta_{0}=\frac{1}{2}+\alpha_{0}, \alpha_{0} \in\left(0, \frac{1}{6}\right]$.

Proof. Suppose that the conclusion is not true. Then, there exists $\left\{v_{n}\right\} \subset S_{L^{\perp}}$ such that $\left\|v_{n}-v\right\| \leq 1, v_{n} \rightarrow v$ and

$$
\lim _{n \rightarrow \infty} \frac{\left\|p\left(v_{n}\right)-p(v)\right\|}{\left\|v_{n}-v\right\|^{\beta_{0}}}=+\infty
$$

On the other hand,

$$
\begin{aligned}
& \left|t_{n}-t\right|=\left\|\left(t_{n}-t\right) v_{n}\right\| \geq\left\|\left(t_{n}-t\right) v_{n}+t\left(v_{n}-v\right)\right\|-\left\|t\left(v_{n}-v\right)\right\| \\
= & \left\|t_{n} v_{n}-t v\right\|-t\left\|v_{n}-v\right\|=\left\|p\left(v_{n}\right)-p(v)\right\|-t\left\|v_{n}-v\right\|,
\end{aligned}
$$

where $p\left(v_{n}\right)=t_{n} v_{n}$ and $t_{n}>0$. Hence, we have

$$
\lim _{n \rightarrow \infty} \frac{\left|t_{n}-t\right|}{\left\|v_{n}-v\right\|^{\beta_{0}}}=+\infty .
$$

It is obvious that there are two cases. The first is there is $\left\{A_{2}^{n_{l}} \cup A_{3}^{n_{l}} \cup B_{3}^{n_{l}}\right\}$ such that $A_{2}^{n_{l}} \cup A_{3}^{n_{l}} \cup B_{3}^{n_{l}} \neq \emptyset$ and the second is there is $\left\{A_{2}^{n_{l}} \cup A_{3}^{n_{l}} \cup B_{3}^{n_{l}}\right\}$ such that $A_{2}^{n_{l}} \cup A_{3}^{n_{l}} \cup B_{3}^{n_{l}}=\emptyset$.

For the first case, by Lemma 4.10, there are two subcases. The first one is there is $\left\{A_{2}^{n_{l^{\prime}}} \cup A_{3}^{n_{l^{\prime}}} \cup B_{3}^{n_{l^{\prime}}}\right\}$ such that $A_{2}^{n_{l^{\prime}}} \cup A_{3}^{n_{l^{\prime}}} \cup B_{3}^{n_{l^{\prime}}} \neq \emptyset$ and $t>t_{n_{l^{\prime}}}$ and the second one is there is $\left\{A_{2}^{n_{l^{\prime}}} \cup A_{3}^{n_{l^{\prime}}} \cup B_{3}^{n_{l^{\prime}}}\right\}$ such that $A_{2}^{n_{l^{\prime}}} \cup A_{3}^{n_{l^{\prime}}} \cup B_{3}^{n_{l^{\prime}}} \neq \emptyset, t<t_{n_{l^{\prime}}}$ and

$$
\int_{\Omega_{n_{l^{\prime}}}}\left|t_{n_{l^{\prime}}} v_{n_{l^{\prime}}}(x)\right| d x \leq C s_{n_{l^{\prime}}}^{\beta_{0}}
$$

where $\Omega_{n_{l^{\prime}}}=A_{2}^{n_{l^{\prime}}} \cup A_{3}^{n_{l^{\prime}}} \cup B_{3}^{n_{l^{\prime}}}, C>0$ is a constant, $v_{n_{l^{\prime}}}=\frac{1}{\sqrt{1+s_{n_{l^{\prime}}}^{2}}}\left(v-s_{n_{l^{\prime}}} d_{n_{l^{\prime}}}\right)$, $\left\|d_{n_{l^{\prime}}}\right\|=1,\left\langle d_{n_{l^{\prime}}}, v\right\rangle=0$ and $s_{n_{l^{\prime}}} \geq 0$, and $\left\{n_{l^{\prime}}\right\}$ is a subsequence of $\left\{n_{l}\right\}$. By Lemma 4.14 and Lemma 4.15, there is a constant $\bar{L}_{h}>0$ such that

$$
\left|t_{n_{l^{\prime}}}-t\right| \leq \bar{L}_{h}\left\|v_{n_{l^{\prime}}}-v\right\|^{\beta_{0}} .
$$

This is a contradiction to (4.42).

For the second case, by Lemma 4.16, there is a constant $\hat{L}_{h}>0$ such that

$$
\left|t_{n_{l}}-t\right| \leq \hat{L}_{h}\left\|v_{n_{l}}-v\right\|^{\beta_{0}} \text {. }
$$

This is also a contradiction to (4.42).

By Theorem 4.17, a conclusion on super-linear property of peak selection to $J$ (4.1) with $f(x, t)$ (4.2) can be verified. 
Theorem 4.18. Assume that $L=\{0\}, f(x, t)$ is in (4.2), $p$ is a peak selection of $J$ (4.1) defined on $S_{L^{\perp}}, p(w)=t_{w} w, t_{w}>0$ for $w \in S_{L^{\perp}}$ and $p$ is continuous at $v \in S_{L^{\perp}}$. Then, $p$ has super-linear property at $v$.

Proof. For every $\left\{v_{n}\right\} \subset S_{L^{\perp}}$ such that $\left\|v_{n}-v\right\| \leq 1, v_{n} \rightarrow v$, denote $p\left(v_{n}\right)=t_{n} v_{n}$, $t_{n}>0$ and $p(v)=t v, t>0$. By Theorem 4.17 there is a constant $L_{h}>0$ such that, for large $n$,

$$
\left\|p\left(v_{n}\right)-p(v)\right\| \leq L_{h}\left\|v_{n}-v\right\|^{\beta_{0}},
$$

$\beta_{0}=\frac{1}{2}+\alpha_{0}, \alpha_{0} \in\left(0, \frac{1}{6}\right]$. On the other hand,

$$
\begin{aligned}
\frac{1}{2} \int_{\Omega}|\nabla w|^{2} d x-\frac{1}{2} \int_{\Omega}|\nabla u|^{2} d x & =\langle\nabla u, \nabla w-\nabla u\rangle+\frac{1}{2} \int_{\Omega}|\nabla w-\nabla u|^{2} d x \\
& =-\langle\Delta u, w-u\rangle+\frac{1}{2}\|w-u\|^{2} .
\end{aligned}
$$

Then, by Remark 2.9, $F(w ; u, z)=\frac{1}{2}\|w-u\|^{2}$ is an upper-bound functional around $u$ for $z \in \partial J(u)$.

Thus, by (4.43),

$$
\lim _{n \rightarrow \infty} \frac{F\left(p\left(v_{n}\right) ; p(v), z\right)}{\left\|v_{n}-v\right\|}=\lim _{n \rightarrow \infty} \frac{\left\|p\left(v_{n}\right)-p(v)\right\|^{2}}{2\left\|v_{n}-v\right\|}=0
$$

i.e., $p$ has super-linear property at $v$.

Remark 4.19. To our numerical example, Lemma 4.1. Lemma 4.3. Lemma 4.5 and Theorem 4.18 guarantee that the peak selection $p(v)=t v$, where $t>0$ and $J(t v)=\max _{s \geq 0} J(s v)$, has super-linear property on $S_{L^{\perp}}$, where $L=\{0\}$.

Now, we start to discuss the details on numerical computation. To carry out the minimax algorithm, we need to find $z_{n}^{k}$ to construct a descent direction. Thus, we have to solve the linear equation

$$
\left\{\begin{array}{l}
\Delta z(x)=-\Delta p\left(v_{n}^{k}\right)(x)-\zeta_{n}^{k}(x), x \in \Omega, \\
\left.z(x)\right|_{x \in \partial \Omega}=0
\end{array}\right.
$$

where $\zeta_{n}^{k} \in \partial G\left(p\left(v_{n}^{k}\right)\right)$ and $p$ is a peak selection to the variational functional $J$ (4.1). By Theorem 1.3, it is still hard to find $\zeta_{n}^{k} \in \partial G\left(p\left(v_{n}^{k}\right)\right)$ since the inclusion $\partial G\left(p\left(v_{n}^{k}\right)\right)(x) \subseteq\left[\bar{f}\left(x, p\left(v_{n}^{k}\right)(x), \hat{f}\left(x, p\left(v_{n}^{k}\right)(x)\right)\right]\right.$ offers little information on $\partial G\left(p\left(v_{n}^{k}\right)\right)$. For nice $f(x, t)$, Theorem 1.5 gives us an equality

$$
\begin{aligned}
\partial G(u)=\{\zeta: \Omega \rightarrow & \mathbb{R} \mid \zeta \text { is measurable, } \zeta(x) \\
& \in[f(x, u(x)-0), f(x, u(x)+0)] \forall x \in \Omega\} .
\end{aligned}
$$

Indeed, we have the following simple lemma.

Lemma 4.20. If $f(x, t)$ is a Baire-measurable function defined on $\Omega \times \mathbb{R}$, is nondecreasing in $t$ and satisfies

$$
|f(x, t)| \leq C_{1}+C_{2}|t|^{\sigma}
$$

where $0<\sigma<\frac{m+2}{m-2}$ for $m \geq 3, \sigma>0$ for $m=1,2$ and $C_{1}, C_{2}>0$ are two constants, then we have that, in $H_{0}^{1}(\Omega)$, for $v \in S_{L^{\perp}}, \zeta \in \partial G(p(v))$ and $(\Delta p(v)+$ $\zeta) \perp[L, v]$ is equivalent to $\zeta$ is a solution to the convex optimization problem

$$
\min _{\eta \in \mathcal{D}} \mathcal{L}(\eta)=\sum_{i=1}^{n-1}\left(\int_{\Omega}(\Delta p(v)+\eta) u_{i} d x\right)^{2}+\left(\int_{\Omega}(\Delta p(v)+\eta) v d x\right)^{2},
$$


where $\mathcal{D}=\{\eta: \Omega \rightarrow \mathbb{R} \mid \eta$ is measurable, $\eta(x) \in[f(x, p(v)(x)-0), f(x, p(v)(x)+$ $0)] \forall x \in \Omega\}$ and $p$ is a peak selection to the variational functional $J$ (4.1) w.r.t. a finite dimensional subspace $L \subset H_{0}^{1}(\Omega)$.

Proof. If $\zeta$ satisfies $\zeta \in \partial G(p(v))$ and $(\Delta p(v)+\zeta) \perp[L, v]$, then, by Theorem 1.5. $\zeta \in$ $\mathcal{D}$ and $\mathcal{L}(\zeta)=0$, i.e., $\zeta$ is a solution to the convex optimization problem (4.45). If $\zeta$ is a solution to the convex optimization problem (4.45), then, $\zeta \in \mathcal{D}$, i.e., $\zeta \in \partial G(p(v))$ by Theorem 1.5. On the other hand, Lemma 2.2 says $\min _{\eta \in \mathcal{D}} \mathcal{L}(\eta)=0$, i.e., $(\Delta p(v)+\zeta) \perp[L, v]$. Hence, $\zeta$ satisfies $\zeta \in \partial G(p(v))$ and $(\Delta p(v)+\zeta) \perp[L, v]$.

To $L=\{0\}$, we have a conclusion as follows.

Lemma 4.21. Assume that $f(x, t)=g(x) h(t)$ is a Baire-measurable function defined on $\Omega \times \mathbb{R}, 0 \leq g(x) \leq C$ in $\Omega$ for constant $C$, and $h(t)$ is nondecreasing in $t$ and satisfies

$$
|h(t)| \leq C_{1}+C_{2}|t|^{\sigma},
$$

for $t \in \mathbb{R}$, where $0<\sigma<\frac{m+2}{m-2}$ for $m \geq 3, \sigma>0$ for $m=1,2$ and $C_{1}, C_{2}>0$ are two constants. If $h(t)$ is discontinuous at $t_{1}, \ldots, t_{k}$ and continuous elsewhere, then, for $v \in H_{0}^{1}(\Omega)$ with $\|v\|=1$, there are $d_{i} \in\left[h\left(t_{i}-0\right), h\left(t_{i}+0\right)\right], i=1, \ldots, k$, such that $\zeta \in \partial G(p(v))$ and $(\Delta p(v)+\zeta) \perp v$, where

$$
\zeta(x)= \begin{cases}g(x) h(p(v)(x)), & \text { if } p(v)(x) \notin\left\{t_{1}, \ldots, t_{k}\right\}, \\ g(x) d_{i}, & \text { if } p(v)(x)=t_{i}, i=1, \ldots, k,\end{cases}
$$

$p$ is a peak selection to the variational functional $J$ (4.1) and $p(v) \neq 0$.

Proof. Denote $p(v)=k(v) v, k(v) \in \mathbb{R}$. Without loss of generality, we assume $k(v)>0$. By Lemma 2.2, there is a $z \in \partial G(p(v))$ such that $(\Delta p(v)+z) \perp v$. Thus, by Theorem 1.5 .

$$
\zeta_{0}^{+}(x)= \begin{cases}g(x) h(p(v)(x)), & \text { if } p(v)(x) \notin\left\{t_{1}, \ldots, t_{k}\right\} \\ g(x) h\left(t_{i}+0\right), & \text { if } p(v)(x)=t_{i} \geq 0 \\ g(x) h\left(t_{i}-0\right), & \text { if } p(v)(x)=t_{i}<0\end{cases}
$$

and

$$
\zeta_{0}^{-}(x)= \begin{cases}g(x) h(p(v)(x)), & \text { if } p(v)(x) \notin\left\{t_{1}, \ldots, t_{k}\right\} \\ g(x) h\left(t_{i}-0\right), & \text { if } p(v)(x)=t_{i} \geq 0 \\ g(x) h\left(t_{i}+0\right), & \text { if } p(v)(x)=t_{i}<0\end{cases}
$$

satisfy $\zeta_{0}^{+}, \zeta_{0}^{-} \in \partial J(p(v))$,

$$
\int_{\Omega}\left(\Delta p(v)+\zeta_{0}^{+}\right) v d x \geq \int_{\Omega}(\Delta p(v)+z) v d x=0
$$

and

$$
\int_{\Omega}\left(\Delta p(v)+\zeta_{0}^{-}\right) v d x \leq \int_{\Omega}(\Delta p(v)+z) v d x=0 .
$$

Then, we can construct $\left\{\zeta_{i}^{+}\right\}$and $\left\{\zeta_{i}^{-}\right\}$such that $\zeta_{i}^{+}, \zeta_{i}^{-} \in \partial J(p(v))$,

$$
\int_{\Omega}\left(\Delta p(v)+\zeta_{i}^{+}\right) v d x \geq 0 \text { and } \int_{\Omega}\left(\Delta p(v)+\zeta_{i}^{-}\right) v d x \leq 0
$$

where

$$
\left\{\begin{array}{l}
\zeta_{i}^{-}=\zeta_{i-1}^{-} \\
\zeta_{i}^{+}=\frac{1}{2}\left(\zeta_{i-1}^{-}+\zeta_{i-1}^{+}\right)
\end{array}\right.
$$


if $\int_{\Omega}\left(\Delta p(v)+\frac{1}{2}\left(\zeta_{i-1}^{-}+\zeta_{i-1}^{+}\right)\right) v d x \geq 0$, or

$$
\left\{\begin{array}{l}
\zeta_{i}^{-}=\frac{1}{2}\left(\zeta_{i-1}^{-}+\zeta_{i-1}^{+}\right) \\
\zeta_{i}^{+}=\zeta_{i-1}^{+}
\end{array}\right.
$$

if $\int_{\Omega}\left(\Delta p(v)+\frac{1}{2}\left(\zeta_{i-1}^{-}+\zeta_{i-1}^{+}\right)\right) v d x \leq 0, i=1,2, \ldots$. During this process, if $\int_{\Omega}(\Delta p(v)+$ $\left.\zeta_{i}^{+}\right) v d x=0$ or $\int_{\Omega}\left(\Delta p(v)+\zeta_{i}^{-}\right) v d x=0$, then $\zeta=\zeta_{i}^{+}$or $\zeta=\zeta_{i}^{-}$. Otherwise,

$$
\zeta(x)=\lim _{i \rightarrow \infty} \zeta_{i}^{+}(x)=\lim _{i \rightarrow \infty} \zeta_{i}^{-}(x)
$$

is in (4.46) and satisfies $\zeta \in \partial G(p(v))$ and $(\Delta p(v)+\zeta) \perp v$.

Remark 4.22. The proof gives us a simple iterative way to numerically capture a $\zeta \in \partial G(p(v))$ such that $(\Delta p(v)+\zeta) \perp v$.

It is easy to check that $u=0$ is a minimal point of $J$ (4.1) if $f$ is in (4.2). Among saddle points, people usually pay more attention to Mountain Pass type saddle points. In this paper, we concentrate on computing them. Of course, more saddle points can be captured if symmetry is used. Thus, in our numerical computation, Lemma 4.21 is used for computing $\zeta_{n}^{k}$ and then the Poisson equation (4.44) is solved to get $z_{n}^{k}$ in the algorithm; even or odd symmetry about the origin is used for capturing symmetric saddle points as $\Omega=(-1,1)$ and even or odd symmetry about $x_{1}$-axis, $x_{2}$-axis or the diagonal lines $x_{2}=-x_{1}$ is used to capture symmetric saddle points as $\Omega=(-1,1) \times(-1,1),\left\{\left(x_{1}, x_{2}\right) \in \mathbb{R} \mid x_{1}^{2}+x_{2}^{2}<1\right\}$. To solve the Poisson equation (4.44), the finite element method is employed. Over $5 \times 10^{4}$ elements on $\Omega \subset \mathbb{R}$ and $5 \times 10^{5}$ triangle elements on $\Omega \subset \mathbb{R}^{2}$ are used. The profiles of approximations for saddle points are listed in Figures 1-10. The corresponding values of $J$ (4.1) are listed in captions. Symmetry of saddle point is also pointed out in captions. Then $\min \{\|\zeta\| \mid \zeta \in \partial J(u)\}$ of every approximation $u$ is less than $10^{-3}$. Hence, these approximations are good approximations of saddle points.

We set $\alpha=8, \beta=6$ and $\Omega=(-1,1)$ in Figures 1 and $2, c=1$ in Figure 1 and $c=24.0625$ in Figure 2. For the first and the second in Figures 1 and 2, $r=0$ and in the third and the fourth, $r=4$. Odd symmetry about the origin is used for capturing the second and even symmetry about the origin is used for calculating the fourth in these two figures. We set $\alpha=8, \beta=6$ to Figures $3-10, c=1$ to Figures $3-6, c=\frac{14}{11}$ to Figures 7 and 8 and $c=60$ to Figures 9 and 10. For Figures $3,5,7$, and $8, r=0$ and to Figures $4,6,9$, and $10, r=4$. For the domain, $\Omega=(-1,1) \times(-1,1)$ to Figures $3,4,7$, and 9 and $\Omega=\left\{\left(x_{1}, x_{2}\right) \in \mathbb{R} \mid x_{1}^{2}+x_{2}^{2}<1\right\}$ to Figures 5, 6, 8, and 10. For capturing the third in Figures 3, 6, 7, and 10 and the second in Figures 5, 8, odd symmetry for $x_{2}$-axis is used and for computing the second in Figures 4, 6, 9, and 10, even symmetry for $x_{2}$-axis is used. For computing the second in Figures 3 and 7 and the third in Figures 4 and 9, odd and even symmetry for the line $x_{2}=-x_{1}$ are used respectively. Odd symmetry for $x_{1}$-axis and $x_{2}$-axis are used for the fourth in Figures 3 and 7 and the third in Figures 5 and 8 and even symmetry for $x_{1}$-axis and $x_{2}$-axis are used for the fourth in Figures 4, 6, 9, and 10. $v_{1}^{1}=\frac{v}{\|v\|}$ was used as initial point for calculating the 
first in Figure 7, where

$$
v(x)= \begin{cases}2\left(x_{2}+1\right), & x_{2} \in\left[-1,-\frac{1}{2}\right], x_{1}+x_{2} \leq 0, x_{1}-x_{2} \geq 0 \\ 2\left(1-x_{2}\right), & x_{2} \in\left[\frac{1}{2}, 1\right], x_{1}+x_{2} \geq 0, x_{1}-x_{2} \leq 0 \\ 2\left(x_{1}+1\right), & x_{1} \in\left[-1,-\frac{1}{2}\right], x_{1}+x_{2} \leq 0, x_{1}-x_{2} \leq 0 \\ 2\left(1-x_{1}\right), & x_{1} \in\left[\frac{1}{2}, 1\right], x_{1}+x_{2} \geq 0, x_{1}-x_{2} \geq 0 \\ 1, & \left(x_{1}, x_{2}\right) \in\left[-\frac{1}{2}, \frac{1}{2}\right] \times\left[-\frac{1}{2}, \frac{1}{2}\right]\end{cases}
$$

It is easy to check that

$$
\iint_{\Omega} v^{6}(x) d x=\frac{37}{28} \text { and } \iint_{\Omega}|\nabla v(x)|^{2} d x=12,
$$

where $\Omega=(-1,1) \times(-1,1)$. Denote $\phi(t)=J\left(t v_{1}^{1}\right)$ for $J$ (4.1). Then, we have

$$
\partial \phi(t)=\left\{\left\langle z, v_{1}^{1}\right\rangle \mid z \in \partial J\left(t v_{1}^{1}\right)\right\} .
$$

For the first in Figure 7, by (4.4),

$$
\partial \phi(t)=\left\{-\iint_{\Omega}(\Delta u+\zeta) v_{1}^{1} d x \mid \zeta \text { is measurable, } \zeta(x) \in f_{-}^{+}(x, u(x)) \forall x \in \Omega\right\},
$$

where

$$
f_{-}^{+}(x, u(x))=[f(x, u(x)-0), f(x, u(x)+0)]= \begin{cases}\left\{8 c|u(x)|^{6} u(x)\right\}, & \text { if }|u(x)|>1, \\ \left\{6 c|u(x)|^{4} u(x)\right\}, & \text { if }|u(x)|<1, \\ {[6 c, 8 c],} & \text { if } u(x)=1, \\ {[-8 c,-6 c],} & \text { if } u(x)=-1,\end{cases}
$$

$u=t v_{1}^{1}$ and $c=\frac{14}{11}$. The equality,

$$
\iint_{\Omega}|\nabla v|^{2} d x-c\left(6 \iint_{\Omega} v^{6} d x+\frac{3}{2}\right)=0
$$

means $0 \in \partial \phi(\|v\|)$. As $t$ is less than $\|v\|$ and very close to $\|v\|$,

$$
\begin{aligned}
\frac{d \phi}{d t} & =\frac{1}{t}\left(\iint_{\Omega}\left|\nabla\left(t v_{1}^{1}\right)\right|^{2} d x-6 c \iint_{\Omega}\left(t v_{1}^{1}\right)^{6} d x\right) \\
& >\frac{1}{t}\left(\iint_{\Omega}|\nabla v|^{2} d x-c\left(6 \iint_{\Omega} v^{6} d x+\frac{3}{2}\right)\right)=0
\end{aligned}
$$

and as $t$ is larger than $\|v\|$ and very close to $\|v\|$,

$$
\begin{aligned}
\frac{d \phi}{d t} & =\frac{1}{t}\left(\iint_{\Omega}\left|\nabla\left(t v_{1}^{1}\right)\right|^{2} d x-c\left(6 \iint_{\Omega_{0}}\left(t v_{1}^{1}\right)^{6} d x+8 \iint_{\Omega_{1}}\left(t v_{1}^{1}\right)^{8} d x\right)\right) \\
& <\frac{1}{t}\left(\iint_{\Omega}\left|\nabla\left(t v_{1}^{1}\right)\right|^{2} d x-c\left(6 \iint_{\Omega}\left(t v_{1}^{1}\right)^{6} d x+2 \iint_{\Omega_{1}}\left(t v_{1}^{1}\right)^{8} d x\right)\right) \\
& <\frac{1}{t}\left(\iint_{\Omega}|\nabla v|^{2} d x-c\left(6 \iint_{\Omega} v^{6} d x+\frac{3}{2}\right)\right)=0,
\end{aligned}
$$

where $\Omega_{0}=\left\{x \in \Omega \mid t v_{1}^{1}(x) \leq 1\right\}$ and $\Omega_{1}=\left\{x \in \Omega \mid t v_{1}^{1}(x)>1\right\}$. Thus, $p\left(v_{1}^{1}\right)=v$. The point $p\left(v_{1}^{1}\right)$ is a nonsmooth point of $J$. 


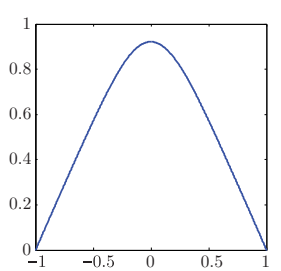

(a)

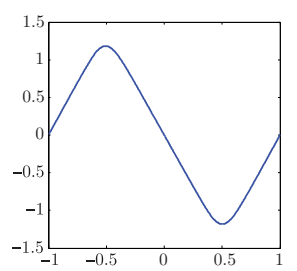

(b)

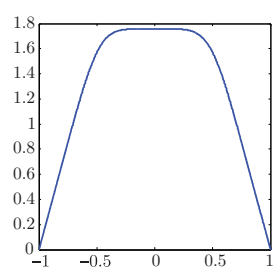

(c)

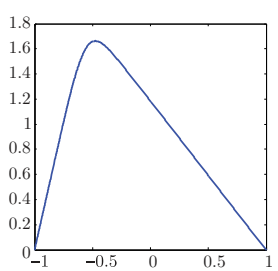

(d)

Figure 1. $c=1, r=0$ for (a) and (b), $r=4$ for (c) and (d). (a) $J=0.6342$, positive saddle point, (b) $J=4.4818$, saddle point odd symmetric about the origin, (c) $J=2.9981$, positive saddle point, (d) $J=3.9094$, saddle point even symmetric about the origin.

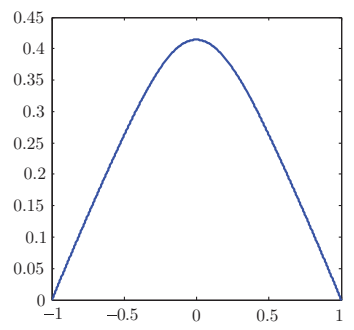

(a)

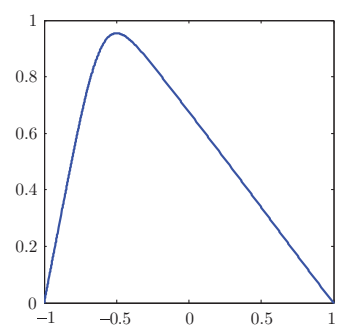

(c)

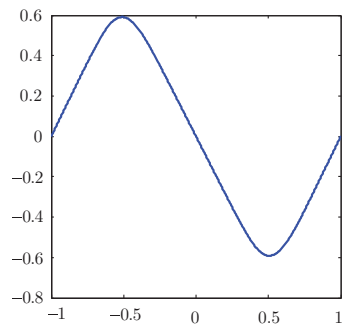

(b)

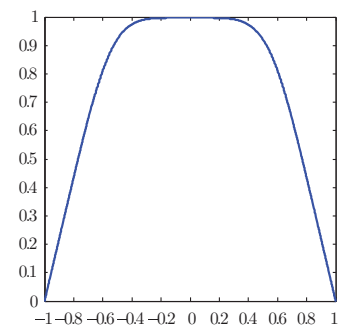

(d)

Figure 2. $c=24.0625, r=0$ for (a) and (b), $r=4$ for (c) and (d). (a) $J=0.1296$, positive saddle point, (b) $J=1.0331$, saddle point odd symmetric about the origin, (c) $J=0.9372$, positive saddle point, (d) $J=1.2156$, saddle point odd symmetric about the origin. 


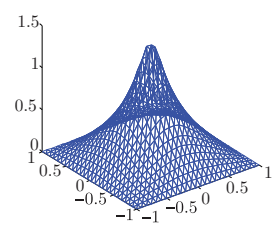

(a)

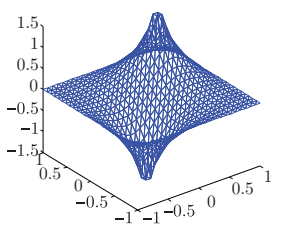

(b)

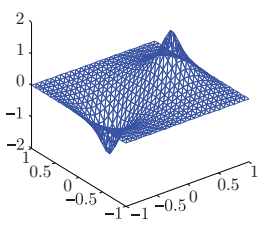

(c)

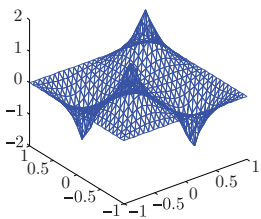

(d)

FiguRE 3. $c=1, r=0$. (a) $J=1.5746$, positive saddle point, (b) $J=4.3662$, saddle point odd symmetric for $x_{2}=-x_{1}$, (c) $J=$ 4.5596, saddle point odd symmetric for $x_{1}=0$ (d) $J=10.1556$, saddle point odd symmetric for $x_{1}=0$ and $x_{2}=0$.

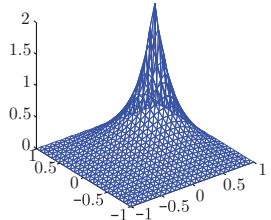

(a)

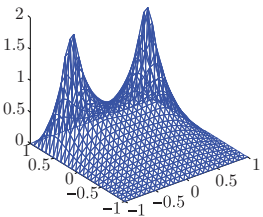

(b)

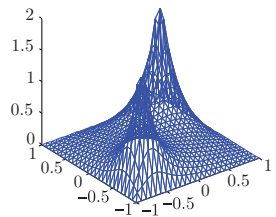

(c)

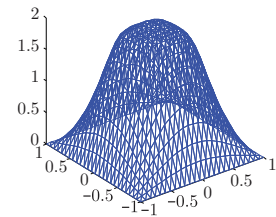

(d)

Figure 4. $c=1, r=4$. (a) $J=3.0167$, positive saddle point, (b) $J=5.8567$, saddle point even symmetric for $x_{1}=0$, (c) $J=$ 5.9545 , saddle point odd symmetric for $x_{2}=-x_{1}$, (d) $J=9.2199$, saddle point even symmetric for $x_{1}=0$ and $x_{2}=0$.

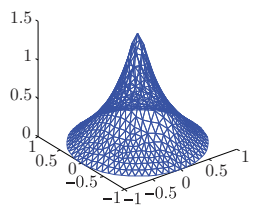

(a)

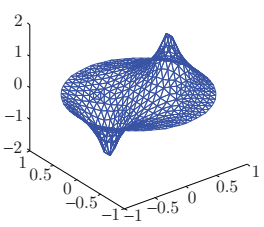

(b)

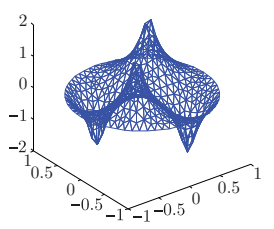

(c)

Figure 5. $c=1, r=0$. (a) $J=1.6614$, positive saddle point, (b) $J=4.7202$, saddle point odd symmetric for $x_{1}=0$, (c) $J=$ 11.2632, saddle point odd symmetric for $x_{1}=0$ and $x_{2}=0$. 


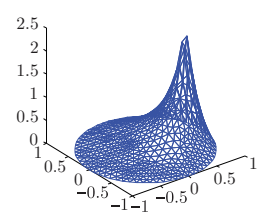

(a)

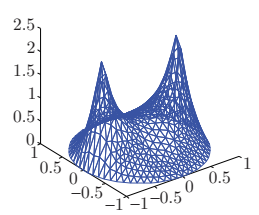

(b)

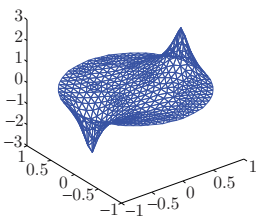

(c)

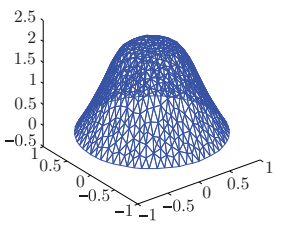

(d)

Figure $6 . c=1, r=4$. (a) $J=4.2512$, positive saddle point, (b) $J=8.1264$, saddle point even symmetric for $x_{1}=0$, (c) $J=$ 8.8090, saddle point odd symmetric for $x_{1}=0$, (d) $J=10.1558$, saddle point even symmetric for $x_{1}=0$ and $x_{2}=0$.

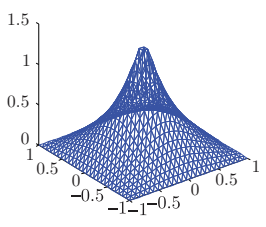

(a)

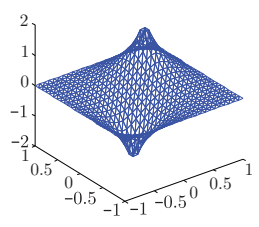

(b)

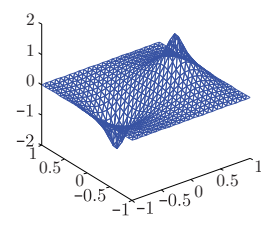

(c)

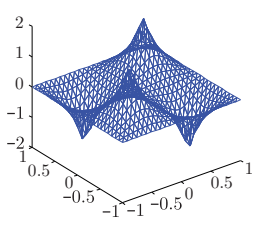

(d)

FiguRE 7. $c=\frac{14}{11}, r=0$. (a) $J=1.4439$, positive saddle point, (b) $J=4.0388$, saddle point odd symmetric for $x_{2}=-x_{1}$, (c) $J=4.1995$, saddle point odd symmetric for $x_{1}=0$ (d) $J=9.3593$, saddle point odd symmetric for $x_{1}=0$ and $x_{2}=0$.

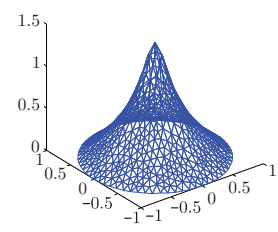

(a)

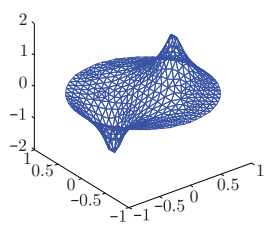

(b)

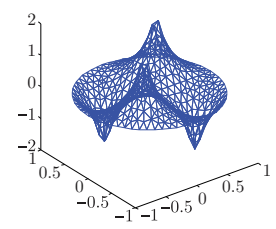

(c)

FiguRE 8. $c=\frac{14}{11}, r=0$. (a) $J=1.5250$, positive saddle point, (b) $J=4.3483$, saddle point odd symmetric for $x_{1}=0$ (c) $J=$ 10.3838 , saddle point odd symmetric for $x_{1}=0$ and $x_{2}=0$. 


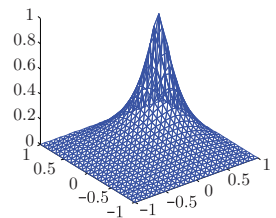

(a)

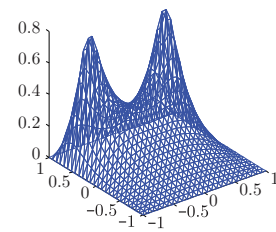

(b)

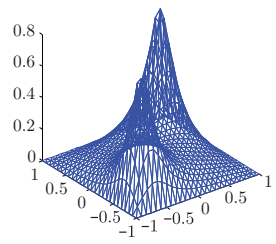

(c)

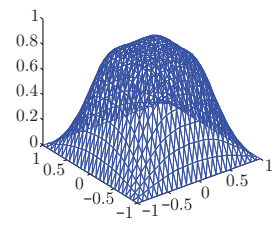

(d)

Figure 9. $c=60, r=4$. (a) $J=0.6098$, positive saddle point, (b) $J=1.1663$, saddle point even symmetric for $x_{1}=0$ (c) $J=$ 1.1958 , saddle point even symmetric for $x_{2}=-x_{1}$, (d) $J=1.8055$, saddle point even symmetric for $x_{1}=0$ and $x_{2}=0$.

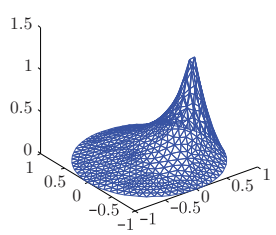

(a)

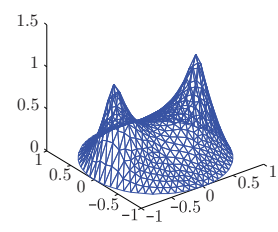

(b)

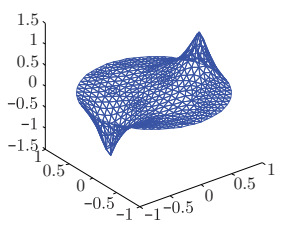

(c)

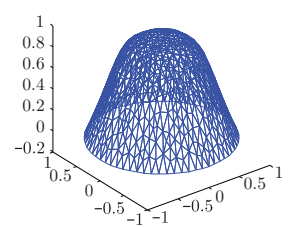

(d)

Figure 10. $c=60, r=4$. (a) $J=1.0044$, positive saddle point, (b) $J=1.8906$, saddle point even symmetric for $x_{1}=0$ (c) $J=$ 2.1015 , saddle point odd symmetric for $x_{1}=0$ (d) $J=2.2920$, saddle point even symmetric for $x_{1}=0$ and $x_{2}=0$.

\section{ACKNowledgment}

The author would like to thank two anonymous referees for their helpful suggestions.

\section{REFERENCES}

1. Robert Alexander Adams and John J. F. Fournier, Sobolev Spaces, Academic Press, New York, 2003. MR.2424078 (2009e:46025)

2. A. Ambrosetti and P. H. Rabinowitz, Dual variational methods in critical point theory and applications, J. Funct. Anal., 14 (1973), 349-381. MR0370183 (51:6412)

3. H. Brezis and L. Nirenberg, Remarks on finding critical points, Commun. Pure Appl. Math., 44 (1991), 939-963. MR1127041 (92i:58032)

4. K. C. Chang, The obstacle problem and partial differential equations with discontinuous nonlinearities, Comm. Pure Appl. Math., 33 (1980), 117-146. MR.562547 (81f:35032)

5. K. C. Chang, Variational methods for nondifferentiable functionals and their applications to partial differential equations, J. Math. Anal. Appl., 80 (1981), 102-129. MR614246 (82h:35025)

6. G. Chen, W. Ni and J. Zhou, Algorithms and visualization for solutions of nonlinear elliptic equations Part I: Dirichlet problems, Int. J. Bifurcation \& Chaos, 7 (2000), 1565-1612. MR.1780923

7. F. H. Clarke, Optimization and Nonsmooth Analysis, Wiley, New York, 1983. MR709590 (85m:49002) 
8. Dimitrios Kandilakis, Nikolaos C. Kourogenis and Nikolaos S. Papageorgiou, Two nontrival critical points for nonsmooth functionals via local linking and applications, J. Global. Optim., 34 (2006), 219-244. MR2210278 (2007b:35081)

9. Nikolaos C. Kourogenis and Nikolaos S. Papageorgiou, Nonsmooth critical point theory and nonlinear elliptic equations at resonance, J. Aust. Math. Soc., A 69 (2000), 245-271. MR.1775181 (2001m:35078)

10. Y. Li and J. Zhou, A minimax method for finding multiple critical points and its applications to nonlinear PDEs, SIAM J. Sci. Comput., 23 (2001), 840-865. MR1860967 (2002h:49012)

11. Y. Li and J. Zhou, Convergence results of a local minimax method for finding multiple critical points, SIAM J. Sci. Comput., 24 (2002), 865-885. MR1950515 (2004a:58013)

12. M. Struwe, Variational Methods, Springer-Verlag, New York, 1996. MR.1411681 (98f:49002)

13. X. Yao and J. Zhou, A local minimax characterization for computing multiple nonsmooth saddle critical points, Math. Program., 104 (2005), no. 2-3, Ser. B, 749-760. MR2179258 (2006k:90166)

14. X. Yao and J. Zhou, A minimax method for finding multiple critical points in Banach spaces and its application to quasi-linear elliptic PDE, SIAM J. Sci. Comp., 26 (2005), 1796-1809. MR2142597 (2006a:58014)

15. X. Yao and J. Zhou, Unified convergence results on a minimax algorithm for finding multiple critical points in Banach spaces, SIAM J. Num. Anal., 45 (2007), 1330-1347. MR2318815 (2009c:65139)

Department of Mathematic, Shanghai Normal University, Shanghai, China 200234

E-mail address: xdyao@shnu.edu.cn 